ARTICLE

\title{
Synapse type-specific proteomic dissection identifies lgSF8 as a hippocampal CA3 microcircuit organizer
}

Nuno Apóstolo (10 1,2, Samuel N. Smukowski ${ }^{3}$, Jeroen Vanderlinden ${ }^{1,2}$, Giuseppe Condomitti,2, Vasily Rybakin ${ }^{4}$, Jolijn ten Bos (1) 1,2, Laura Trobiani ${ }^{5}$, Sybren Portegies ${ }^{1,2}$, Kristel M. Vennekens ${ }^{1,2}$, Natalia V. Gounko (1) 1,2,6, Davide Comoletti ${ }^{5,7}$, Keimpe D. Wierda ${ }^{1,2}$, Jeffrey N. Savas (iD ${ }^{3 凶} \&$ Joris de Wit (iD ${ }^{1,2}{ }^{凶}$

Excitatory and inhibitory neurons are connected into microcircuits that generate circuit output. Central in the hippocampal CA3 microcircuit is the mossy fiber (MF) synapse, which provides powerful direct excitatory input and indirect feedforward inhibition to CA3 pyramidal neurons. Here, we dissect its cell-surface protein (CSP) composition to discover novel regulators of MF synaptic connectivity. Proteomic profiling of isolated MF synaptosomes uncovers a rich CSP composition, including many CSPs without synaptic function and several that are uncharacterized. Cell-surface interactome screening identifies IgSF8 as a neuronal receptor enriched in the MF pathway. Presynaptic Igsf8 deletion impairs MF synaptic architecture and robustly decreases the density of bouton filopodia that provide feedforward inhibition. Consequently, IgSF8 loss impairs excitation/inhibition balance and increases excitability of CA3 pyramidal neurons. Our results provide insight into the CSP landscape and interactome of a specific excitatory synapse and reveal IgSF8 as a critical regulator of CA3 microcircuit connectivity and function.

\footnotetext{
${ }^{1}$ VIB Center for Brain \& Disease Research, Herestraat 49, 3000 Leuven, Belgium. ${ }^{2}$ KU Leuven, Department of Neurosciences, Leuven Brain Institute, Herestraat 49, 3000 Leuven, Belgium. ${ }^{3}$ Department of Neurology, Northwestern University Feinberg School of Medicine, Chicago, IL 60611, USA. ${ }^{4}$ Immunobiology, REGA Institute, Department of Microbiology and Immunology, KU Leuven, Leuven, Belgium. ${ }^{5}$ School of Biological Sciences, Victoria University of Wellington, Wellington 6140, New Zealand. ${ }^{6}$ Electron Microscopy Platform \& VIB Biolmaging Core, Herestraat 49, 3000 Leuven, Belgium. ${ }^{7}$ Child Health Institute of New Jersey, and Departments of Neuroscience and Cell Biology, Robert Wood Johnson Medical School, Rutgers University, New Brunswick, NJ 08901, USA.凶email: jeffrey.savas@northwestern.edu; joris.dewit@kuleuven.vib.be
} 
E: xcitatory and inhibitory neurons are connected into microcircuits and dynamically interact to process information. The specific patterns of connectivity between the neurons in a microcircuit, and the properties and plasticity of transmission at their synapses, are key factors in generating circuit output, from motor to cognitive function ${ }^{1-3}$. Elucidating the molecular mechanisms controlling microcircuit connectivity is important for understanding circuit function in physiology and disease.

The hippocampal mossy fiber (MF) synapse connecting dentate gyrus (DG) granule cells (GCs) and CA3 pyramidal neurons (Fig. 1a) is a central synapse in the CA3 microcircuit that is critical for hippocampal function ${ }^{4}$. A giant MF presynaptic bouton engulfs a postsynaptic element consisting of multiheaded dendritic spines, or thorny excrescences ${ }^{5-7}$. Each MF bouton contains a large vesicle pool and multiple release sites capable of providing powerful excitatory input to CA3 pyramidal neurons. This robust excitation is controlled by strong feedforward inhibition of CA3 pyramidal neurons ${ }^{8}$, mediated by filopodia extending from the MF bouton that form excitatory synapses onto interneurons in stratum lucidum $(\mathrm{SL})^{9}$ (Fig. 1a). SL interneurons in turn provide inhibition to CA3 pyramidal neurons. As MF-interneuron synapses are more numerous than MF-CA3 synapses $^{9}$, this results in net inhibition of CA3 pyramidal neurons. The net inhibition changes to excitation upon an increase in GC firing frequency, due to a rapid depression of the MFinterneuron synapse and a strong facilitation of the MF-CA3 synapse ${ }^{10,11}$. Together, the connectivity and synaptic plasticity properties of the CA3 microcircuit control CA3 pyramidal neuron excitability and firing properties ${ }^{12,13}$. Upon learning, GCs recruit feedforward inhibition through increased connectivity of MF filopodia with fast-spiking SL interneurons, which is important for memory precision ${ }^{14,15}$. Conversely, feedforward inhibition decreases with aging, resulting in CA3 hyperactivity ${ }^{16}$. Precise control of CA3 microcircuit connectivity and function is thus critical for cognitive function.

Cell-surface proteins (CSPs), including transmembrane, membrane-anchored, and secreted proteins, play a key role in the formation of precise connectivity ${ }^{17-19}$. CSPs are expressed in cell type-specific combinations ${ }^{20-23}$ and form protein-protein interaction networks that regulate circuit assembly ${ }^{24-26}$. Recent studies reveal a synaptic input-specific localization and function of postsynaptic CSPs in hippocampal pyramidal neuron dendrites ${ }^{27-30}$. Interneuron cell type-specific expression of presynaptic CSPs regulates domain-specific innervation of cortical pyramidal neurons ${ }^{31}$. Together, synaptic input-specific distribution of CSPs in dendrites and differential expression in axonal populations shape circuit connectivity ${ }^{32}$. Comprehensive characterization of the CSP composition of specific connections has remained challenging, however, and the cell-surface interactions regulating CA3 microcircuit connectivity remain poorly understood.

Here, we dissect the CSP composition of the MF synapse to discover novel regulators of synaptic connectivity in the CA3 microcircuit. Using mass spectrometry (MS) to profile the proteome of isolated MF synaptosomes, we identify a rich CSP repertoire that includes adhesion proteins, guidance cue receptors, extracellular matrix (ECM) proteins, and several uncharacterized CSPs. Approximately $80 \%$ of these CSPs has not been reported to localize or function at MF synapses and $~ 50 \%$ lacks an annotated synaptic function. Combining proteome and CSP interactome screening with extensive validation identifies IgSF8 as an uncharacterized neuronal receptor strongly enriched in the MF pathway. IgSF8 localizes to both MF bouton and filopodia. Dentate GC-specific deletion of $I g s f 8$ reduces the number of release sites in MF boutons and robustly decreases the number of filopodia. As a consequence, feedforward inhibition of CA3 pyramidal neurons is strongly reduced and excitability is increased, indicating that IgSF8 is required to maintain excitation/inhibition balance and control excitability in the CA3 microcircuit. Taken together, our results provide insight into the CSP landscape and interactome of a specific excitatory synapse type, uncover multiple uncharacterized synaptic CSPs, and reveal IgSF8 as a regulator of CA3 microcircuit connectivity and function.

\section{Results}

Isolation of MF synaptosomes. To isolate MF synapses and start uncovering their CSP composition, we relied on two key features of the MF synapse: its large size and the presence of puncta adherentia (PA), a specialized type of adhesive junction found at large synapses ${ }^{33}$ that is morphologically and molecularly distinct from the synaptic junction. We combined two established approaches to take advantage of these features. First, we prepared MF synaptosomes from postnatal (P) day 28 hippocampal homogenate, a time point at which MF synapses have matured ${ }^{34}$. We used a previously established biochemical enrichment method $^{35}$ that relies on the large size of MF synapses (Supplementary Fig. 1a), and verified that this method enriches for MF synaptosomes. Electron microscopy (EM) analysis showed the presence of large synaptosomes with a presynaptic compartment packed with synaptic vesicles engulfing a postsynaptic compartment (Supplementary Fig. 1b). To confirm the identity of these synaptosomes, we used the MF synapse markers Synaptoporin (Synpr), a presynaptic vesicle-associated protein ${ }^{36}$, and Nectin3, a CA3-enriched (http://mouse.brain-map.org/; https://hipposeq. janelia.org/ $)^{37}$ adhesion protein that localizes to the postsynaptic side of PA junctions in mature MF synapses ${ }^{38}$ (Fig. 1b). As an additional MF synapse marker, we used glutamate receptor ionotropic kainate 5 (GluK5), a predominantly postsynaptically localized kainate receptor enriched at MF synapses ${ }^{39,40}$ (Supplementary Fig. 1c). Immunolabeling revealed the presence of all three MF synaptic markers in MF synaptosomes (Supplementary Fig. 1d). Synpr labeling overlapped with the excitatory presynaptic marker vesicular glutamate transporter 1 (VGluT1), whereas Nectin3 labeling was restricted to small puncta (Supplementary Fig. 1d), as expected for a PA-localized postsynaptic protein. GluK5 labeling was similarly restricted to small puncta (Supplementary Fig. 1d). Quantification showed an enrichment of large Synpr- and Nectin3-positive synaptosomes in the MF synaptosome-containing fraction (Supplementary Fig. 1e). We subsequently accelerated the biochemical enrichment procedure by omitting gradient centrifugation and depleting myelin from the sample (Supplementary Fig. 1f). Second, we subjected the biochemical preparation to fluorescence-activated synaptosome sorting ${ }^{41}$ to deplete nuclei and further enrich for MF synaptic material. To this end, we live-labeled MF synaptosomes with a fluorophore-conjugated monoclonal antibody against the extracellular domain of Nectin3 (Supplementary Fig. 1g) and with FM4-64 membrane dye to label plasma membrane-bound particles (Fig. 1c and Supplementary Fig. 1h, i), and sorted them in a fluorescent cell sorter.

Western blot (WB) analysis showed the presence of MF synapse markers Synpr and Nectin3 in the sorted MF synaptosome sample, whereas myelin and nuclear markers were largely depleted from the sorted fraction (Fig. 1d). Immunofluorescence analysis confirmed the presence of Synpr-, Nectin3-, and VGluT1-positive large synaptosomes in the sorted sample (Fig. 1e, f). In addition to Synpr-/Nectin3-/VGluT1-positive synaptosomes, VGluT1positive particles were invariably present (Fig. 1e, f). Such debris has previously been observed following fluorescent synaptosome 
a
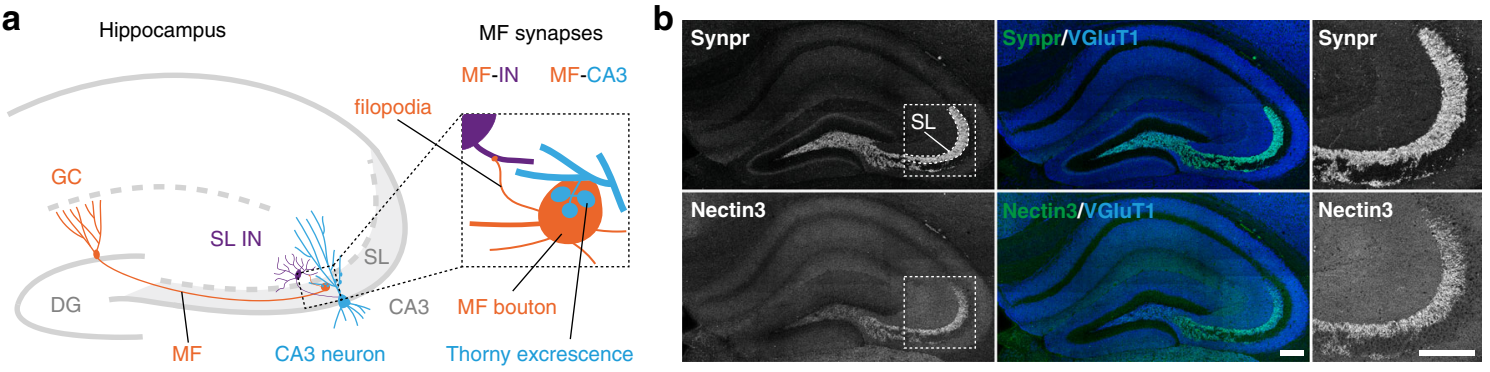

C

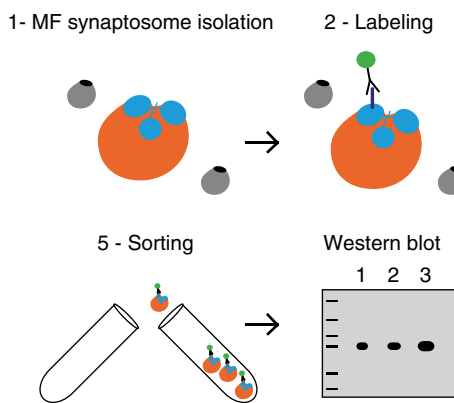

e

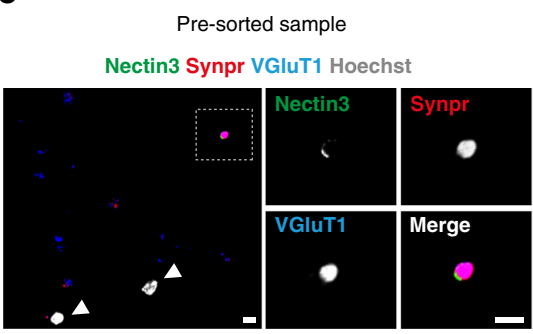

3 - FM4-64 dye

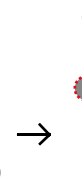

$\rightarrow$

Immunostaining

or
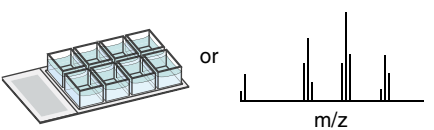

d

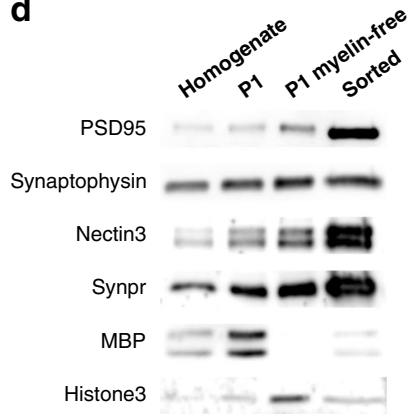

f

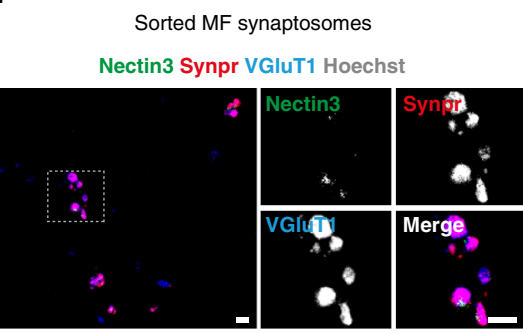

g $M F$

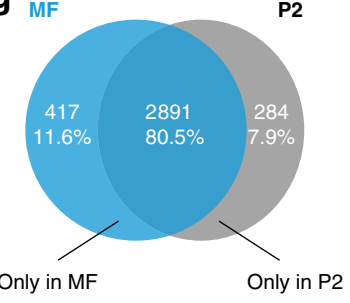

Total of 3592 proteins identified

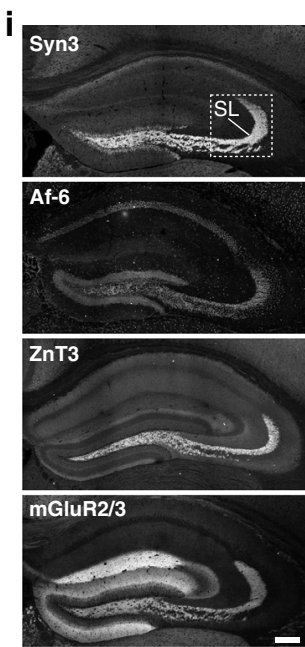

h

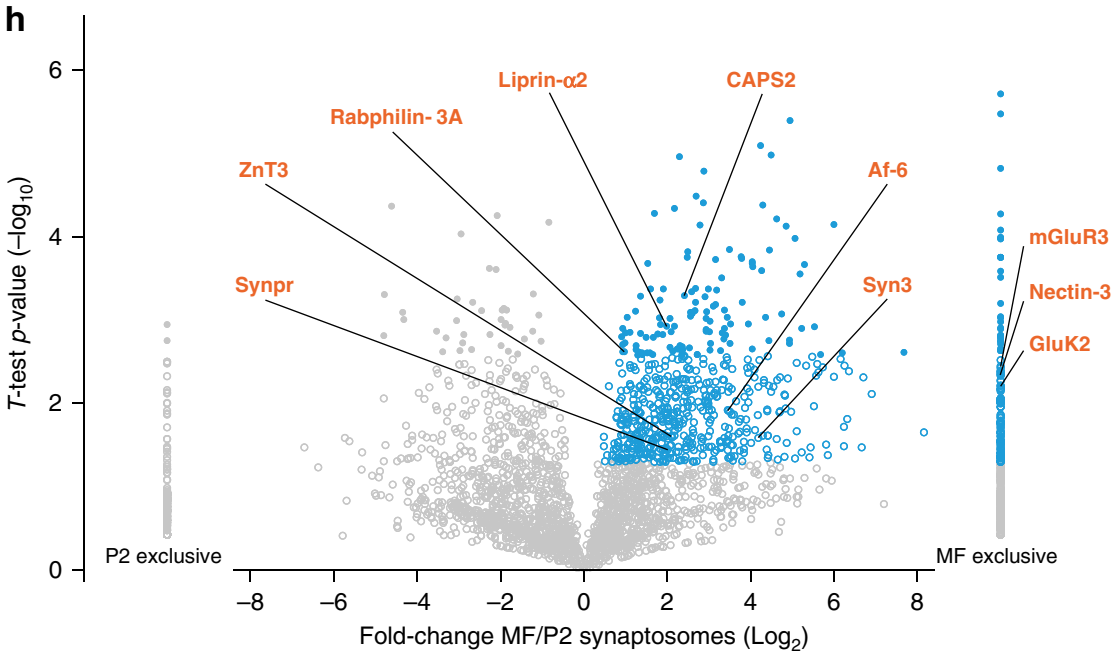

Fig. 1 Isolation and proteomic profiling of MF synaptosomes. a Cartoon illustrating the hippocampal MF synapse. GC neurons, mossy fibers (MFs), MF bouton, and filopodia are in orange. CA3 pyramidal neuron and respective thorny excrescences are in blue. Stratum lucidum (SL) interneuron (IN) is in purple. DG dentate gyrus. b Confocal images of P28 mouse hippocampal sections immunostained for Synpr, Nectin3, and VGluT1. Magnified insets of the $\mathrm{SL}$ in CA3 are shown on the right. $\mathbf{c}$ Workflow to isolate and analyze MF synaptosomes. $\mathbf{d}$ Validation of enrichment for MF synaptosomes in sorted material by western blot. MPB, myelin-binding protein. e, f Confocal images of presorted and sorted material, respectively, immunostained for Nectin3, Synpr, VGluT1, and Hoechst. $\mathbf{g}$ Venn diagram capturing number and distribution of proteins identified in sorted MF synaptosomes and P2 synaptosomes by LC-MS/MS in three independent experiments (10-12 mice per experiment). $\mathbf{h}$ Relative distribution of proteins detected in sorted MF synaptosomes and P2 synaptosomes. Significant proteins with positive MF/P2 synaptosome $\log _{2}$ fold change are highlighted in blue ( $p$ value $\leq 0.05$, two-sided Student's $t$ test). High-confidence measurements at a 5\% FDR are shown as closed circles ( $q$ value $\leq 0.05$, Benjamini-Hochberg correction). A selection of known MF synaptic proteins is annotated in orange. i Confocal images of P28 mouse hippocampal sections immunostained for known MF synaptic markers detected in sorted MF synaptosomes. Source data are provided as a Source data file. Scale bars in $\mathbf{b}$ and $\mathbf{i} 200 \mu \mathrm{m}$, in $\mathbf{e}$ and $\mathbf{f} 5 \mu \mathrm{m}$. 
sorting ${ }^{41}$ and may represent synaptic membrane fragments, resulting from the synaptosome preparation or sorting procedure, indicating that careful validation of potential hits resulting from proteomic analysis of this material is required. Together, these results show that a combination of biochemical enrichment, fluorescent labeling, and sorting successfully enriches for MF synaptosomes from hippocampal tissue homogenate.

Proteomic profiling of MF synaptosomes. For proteomic analysis, we compared sorted MF synaptosomes to P2 synaptosomes isolated in parallel from the same hippocampal homogenate (Supplementary Fig. 1f). P2 synaptosomes represent a mixed population of small hippocampal synapses, and serve as a background reference in our analysis. We analyzed sorted MF synaptosomes and P2 synaptosomes from three independent experiments (10-12 mice per experiment) by liquid chromatography tandem MS (LC-MS/MS)-based proteomics. We identified 3592 proteins with at least three peptide identifications among replicates; 11.6 and $7.9 \%$ of these proteins were exclusively detected in sorted MF synaptosomes or P2 synaptosomes, respectively (Fig. 1g and Supplementary Data 1). Gene ontology (GO) analysis showed a similar enrichment for synaptic terms in sorted MF and P2 synaptosomes (Supplementary Fig. 1j). We calculated $\log _{2}$ fold-change enrichment in sorted MF vs P2 synaptosomes using a label-free semiquantitative approach based on the normalized spectral abundance factor (NSAF; Fig. 1h, Supplementary Fig. 1k and Supplementary Data 1). Our analysis revealed 605 proteins significantly enriched in sorted MF synaptosomes and 138 additional significant proteins exclusively detected in MF synaptosomes (Fig. $1 \mathrm{~h}$ and Supplementary Data 1). This collection comprises multiple proteins previously reported to be strongly enriched at MF synapses, including the synaptic vesicle-associated proteins Synpr, Synapsin3 (Syn3) ${ }^{42}$, Rabphilin-3A $\mathrm{A}^{43}$, and zinc transporter 3 (ZnT3/Slc30a3) ${ }^{44}$; the glutamate receptors GluK2/Grik2 (ref. ${ }^{45}$ ) and metabotropic glutamate receptor 3 (mGluR3/Grm 3$)^{46}$; the presynaptic scaffold protein liprin-a2 (Ppfia2) ${ }^{47}$; the dense core vesicle secretionrelated protein calcium-dependent secretion activator 2 (CAPS2/ Cadps2) ${ }^{48}$; and the PA-associated proteins Nectin3 (listed as Pvrl3 in Supplementary Data 1) and afadin (Af-6/Mllt4) $)^{38}$ (Fig. 1h and Supplementary Fig. 11). Using immunohistochemistry (IHC), we confirmed strongly enriched localization of Syn3, Af-6, mGluR2/3, and ZnT3 in SL (Fig. 1i). Relatively few peptides were detected for Nectin3, the marker used to label MF synaptosomes, which may be due to a low abundance and restricted localization of this protein (Supplementary Fig. 1d). Supporting this notion, similar peptide amounts were detected for GluK5/ Grik5 (Supplementary Data 1), a transmembrane receptor with a restricted localization in MF synaptosomes (Supplementary Fig. 1d). Together, these results indicate that LC-MS/MS analysis of isolated MF synaptosomes confidently identifies MF synaptic proteins.

Dissection of MF synapse CSP composition. Using the UniProt database (https://www.uniprot.org), we next queried our proteomic dataset for transmembrane, membrane-anchored, and secreted proteins among the proteins significantly enriched in sorted MF synaptosomes vs P2 synaptosomes and those exclusively detected in MF synaptosomes. We identified a panel of 77 CSPs, including adhesion proteins, receptors, secreted glycoproteins, receptor protein tyrosine phosphatases, and tyrosine kinases (Fig. 2a and Supplementary Data 2). Most major CSP families, such as the immunoglobulin (Ig) superfamily (IgSF), fibronectin type-III (FN3), and leucine-rich repeat (LRR) family, were represented (Fig. 2b). Only a small proportion (20,8\%) of these MF synapse
CSP candidates has been previously reported to localize or function at MF synapses (Supplementary Data 2). GO analysis using the synapse biology SynGO database (https://syngoportal.org) ${ }^{49}$ revealed that many of the CSPs identified at MF synapses lack a synaptic function (Fig. 2c). The secreted protein bone morphogenic protein/retinoic acid-inducible neural-specific protein 2 (BRINP2), and the transmembrane receptors family with sequence similarity 171 member A2 (FAM171A2), adipocyte plasma membraneassociated protein (APMAP), and IgSF8 lack a known function in the brain altogether (Fig. 2c).

To validate these results, we tested a large panel of antibodies for detection of CSPs by WB and IHC (Supplementary Fig. 2a and Supplementary Data 2). We validated the presence of 15 CSPs in sorted P28 MF synaptosomes by WB (Fig. 2d). Of the SynGO-annotated CSPs, we confirmed the secreted ECM protein TenR; the transmembrane receptors neuropilin-1 (NRP1), receptor-type tyrosine-protein phosphatase delta (RPTP $\delta$ ) and sigma (RPTP $)$, and Teneurin4, as well as the GPI-anchored receptor contactin 1 (CNTN1; Fig. 2d). Of the CSPs lacking a SynGO-annotated synaptic function, we validated the transmembrane receptors intercellular adhesion molecule 5 (ICAM5), IgSF containing LRR protein 2 (ISLR2), Neogenin, PlexinA1, roundabout homolog 2 (ROBO2), mast/stem cell growth factor receptor SCFR/Kit, and the GPI-anchored receptor neural growth regulator 1 (NEGR1; Fig. 2d). Of the uncharacterized CSPs in the brain, we confirmed the presence of BRINP2 and IgSF8 in sorted MF synaptosomes (Fig. 2d).

Using IHC on P28 mouse hippocampal sections, we validated localization to the MF pathway for 21 CSPs, 15 of which showed a striking laminar distribution (Fig. 2e and Supplementary Fig. 2b). We confirmed strong localization to SL for the SynGO-annotated secreted proteins neuronal pentraxin 1 (NPTX1) and neuronal olfactomedin-related ER localized protein/olfactomedin-1 (OLFM1), as well as the transmembrane receptors neural cell adhesion molecule 1 (NCAM1), NRP1 and NRP2, and LRR and FN3 domain-containing protein 1 (LRFN1/SALM2; Fig. 2e). Of the CSPs without synaptic function, the secreted protein cartilage acidic protein 1 (CRTAC1); the transmembrane receptors NCAM2, ISLR2, Neogenin, ROBO2, and PlexinA3; and the GPI-anchored receptor NEGR1 displayed strongly enriched immunoreactivity in SL (Fig. 2e). Of the uncharacterized CSPs, we validated FAM171A2 in SL, which displayed weak labeling in SL, and IgSF8, which showed strong labeling in the MF pathway (Fig. 2e).

Besides these 15 CSPs with lamina-specific localization patterns, we observed a broad hippocampal distribution, including SL, for an additional six CSPs (Supplementary Fig. 2b). Among these are CNTN1 and TenR, which we also confirmed by WB (Fig. 2d). CSPs detected in sorted MF synaptosomes by WB and localized to SL by IHC were in good agreement (Fig. $2 \mathrm{~d}$, e and Supplementary Fig. 2b), with three exceptions: ICAM5, PlexinA1, and SCFR/Kit. While these three CSPs were validated in sorted MF synaptosomes by WB (Fig. 2d), they showed little immunoreactivity in SL (Supplementary Fig. 2c). It is possible that the antibodies used do not recognize the epitopes for these CSPs in SL or that these CSPs are present in MF-sorted synaptosomes, as contaminants and represent false positives. SCFR/Kit, however, has previously been shown to function at MF synapses $^{50-52}$ (Supplementary Data 2). In conclusion, dissection of the MF surface proteome reveals a diverse CSP repertoire containing many CSPs that were not previously shown to be associated with synapses and several CSPs of uncharacterized function.

MF synapse CSP interactome screening. CSPs exert their function by forming protein-protein interaction networks ${ }^{17,24}$ 
a

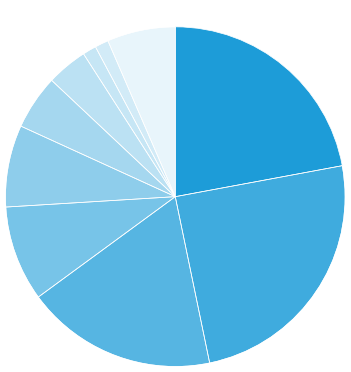

CSP Functional class

- Cell adhesion (17)

- Cell surface receptor (19)

- Synaptic Adhesion (14)

- Receptor tyrosine kinase (7)

- Receptor tyrosine phosphatase (6)

- Extracellular matrix (4)

- Secreted glycoproteins (3)

- Receptor auxiliary protein (1)

G-protein coupled receptor (1)

Uncharacterized (5) b

CSP Family/Domains

C

CSPs with genes not annotated in SynGO

\begin{tabular}{|lll|}
\hline ADAM11 & FAM171A2 $^{*}$ & Neogenin \\
APMAP $^{*}$ & GPR37L1 & Neurofascin \\
BRINP2* $^{*}$ & HEPACAM & Neurotrimin \\
CADM4 & IgSF8 $^{*}$ & Plexin-A1 \\
CD200 & ISLR2 & Plexin-A2 \\
CNTNAP2 & SCFR/Kit & Plexin-A3 \\
CRTAC1 & LINGO1 & RPTP 8 \\
ELFN2 & LRP1 & ROBO1 \\
ELFN1 & LSAMP & ROBO2 \\
Eph-A6 & NCAM2 & SIRP $\alpha$ \\
Eph-B4 & NEGR1 & VCAM1 \\
\hline
\end{tabular}

d

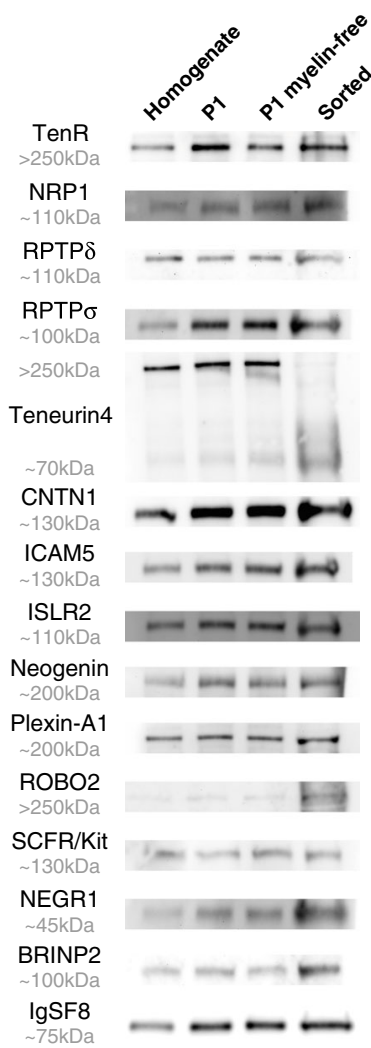

SynGO annotation of CSP genes

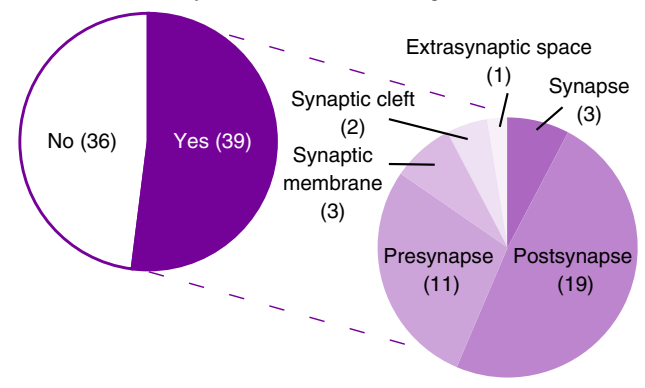

e
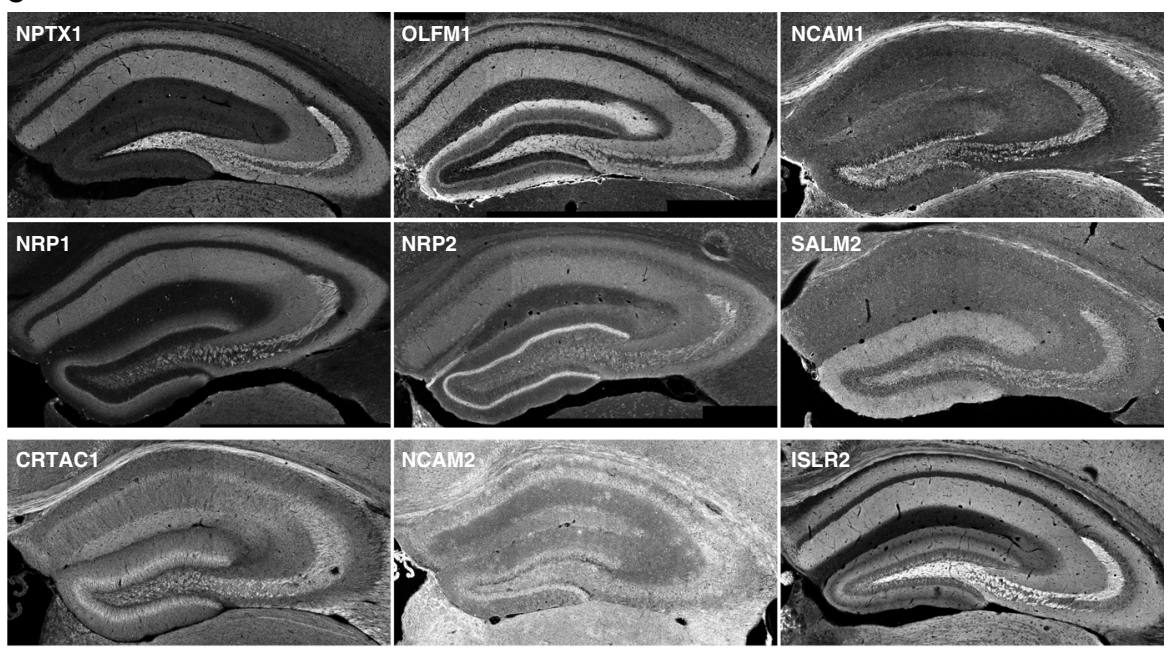

CSPs with genes annotated in SynGO

$\begin{array}{lll}\text { 4F2hc } & \text { NCAM1 } & \text { NRXN3 } \beta \\ \text { ADAM22 } & \text { Nectin3 } & \text { OLFM1 } \\ \text { ADAM23 } & \text { NGL3 } & \text { Plexin-A4 } \\ \text { CNTN1 } & \text { NLGN1 } & \text { Plexin-C1 } \\ \text { CNTNAP1 } & \text { NLGN2 } & \text { RPTP } \alpha \\ \text { Eph-A4 } & \text { NLGN3 } & \text { RPTP } \delta \\ \text { Eph-A7 } & \text { NPTN } & \text { RPTP } \sigma \\ \text { Eph-B2 } & \text { NPTX1 } & \text { SALM2 } \\ \text { HAPLN1 } & \text { NrCAM } & \text { Shisa6 } \\ \text { L1CAM } & \text { NRP1 } & \text { Teneurin4 } \\ \text { LAR } & \text { NRP2 } & \text { TenR } \\ \text { LGl1 } & \text { NRXN1 } \alpha & \text { TrkC } \\ \text { Lphn1 } & \text { NRXN3 } \alpha & \text { VCAN } \\ \text { Lphn3 } & \text { NRXN1 } \beta & \end{array}$


To elucidate ligand-receptor relationships among the CSPs identified in sorted MF synaptosomes and obtain clues about potential roles of the uncharacterized CSPs, we systematically screened for pairwise interactions. We generated a library of constructs containing the extracellular domains of 73 CSPs (see "Methods" section) identified in sorted MF synaptosomes (Fig. 2c and Supplementary Data 2), fused at their C-terminus with human alkaline phosphatase (AP) or the Fc region of human IgG1 (Supplementary Fig. 3a, b). We subsequently generated and validated recombinant AP- and Fc-tagged proteins (Supplementary Data 3 and "Methods" section), and performed a pairwise high-throughput interaction screen using a semiautomated 384-well format enzyme-linked immunosorbent assay (ELISA)based assay ${ }^{25,53,54}$ (Fig. 3a and Supplementary Fig. 3a, b). The screen was designed to test interactions between $73 \mathrm{AP}$ - and Fctagged proteins in both orientations (i.e., AP-X vs Fc-Y and AP-Y vs Fc-X), resulting in $73 \times 73=5329$ experimental points and screening of 2701 unique pairwise interactions. Positive interactions (blue in Fig. 3a) were defined as those wells that exhibited an $\mathrm{OD}_{650}>5$-fold over background values (FOB) and that were not present in every bait. The vast majority were negative wells, which were either completely clear, or had negligible FOB values (white in Fig. 3a). False-positive wells were characterized by having significant signal in all baits.

The CSP interactome screen reproducibly identified two major modules of known ligand-receptor pairs: the synaptic adhesion molecules neurexins (NRXNs) and neuroligins (NLGNs) ${ }^{55}$, and the IgLON IgSF subfamily of synaptic adhesion molecules ${ }^{56}$, confirming specificity of the screen. Multiple additional known interactions were reproducibly identified, including Teneurin4

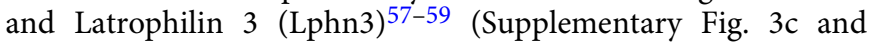
Supplementary Data 3). Combining the results of three independent experiments, using the criteria outlined above and considering only interactions detected at least twice independent of orientation, we identified 38 interacting pairs, 10 of which, to the best of our knowledge, have not been reported before (Fig. 3b and Supplementary Data 3). The interaction of IgSF8 with TenR was among the most reproducible previously unreported ligand-receptor pairs, and IgSF8 was the only uncharacterized CSP for which the interactome screen yielded a binding partner (Fig. 3b and Supplementary Data 3). Thus, CSP interactome analysis identifies multiple ligand-receptor pair modules at MF synapses that may play a role in shaping MF synapse connectivity.

As the MF synapse CSP interactome screen encompassed only those CSPs that reached significance in our proteomic analysis, we next performed affinity chromatography using recombinant IgSF8-ecto-Fc as bait on whole brain synaptosome extract and on MF synaptosome extract to screen a larger panel of prey proteins (Fig. 3c and Supplementary Data 4). Bound proteins were analyzed by LC-MS/MS ${ }^{60}$. We again identified TenR as the main CSP interacting with IgSF8, both in whole brain synaptosome extract, as well as in MF synaptosome extract (Fig. 3d, e and Supplementary Data 4).

To validate the IgSF8-TenR interaction, we used cell-surface binding assays (Fig. 3f) and pull-down assays (Supplementary Fig. 3d) in transfected HEK293T cells, and confirmed the interaction. To test whether IgSF8 and TenR interact directly, we mixed equimolar amounts of Fc control protein, TenR-Fc, or the ECM protein Brevican-Fc, with His-IgSF8 recombinant protein and precipitated Fc proteins. This binding assay showed that the IgSF8-TenR interaction is direct and specific (Fig. 3g). Biolayer interferometry (BLI) using various concentrations of purified IgSF8 and TenR proteins (Supplementary Fig. 3e) determined the dissociation constant $\left(K_{\mathrm{D}}\right)$ for IgSF8-TenR to be $\sim 1.4 \mu \mathrm{M}$ (Fig. 3h, i). Taken together, synapse type-specific proteome dissection combined with CSP interactome screening identifies IgSF8-TenR as a receptor-ligand pair at MF synapses.

IgSF8 localizes to MF boutons and filopodia. The identification of TenR, which regulates synaptic structure, plasticity, and excitability ${ }^{61-63}$, as an IgSF8 ligand suggests a synaptic function for IgSF8. IgSF8 is a type I membrane protein with a large extracellular domain containing four Ig-like C2-type domains, a transmembrane region, and a short cytoplasmic domain (Fig. 4a). This protein is one of four members of an IgSF subfamily containing the Glu-Trp-Ile (EWI) transmembrane motif of unknown biological function. We first assessed IgSF8's protein expression profile and localization. IgSF8 protein levels in hippocampal lysate mildly increased during postnatal development (Fig. 4b). To determine the synaptic localization of IgSF8, we performed subcellular fractionation, as the conditions required for IgSF8 IHC proved unsuitable for high-resolution imaging. IgSF8 mainly distributed to the Triton-soluble fraction of synaptosomes containing the presynaptic protein synaptophysin (Fig. 4c). In addition, two faint bands were observed in the Triton-insoluble fraction containing the postsynaptic density (PSD) protein PSD95. These biochemical results support a predominantly presynaptic localization of IgSF8, but do not exclude a postsynaptic presence. The IgSF8 antibody was specific, as IgSF8 protein levels were largely abolished in lysate of cultured Igsf8 conditional knock out $(\mathrm{cKO})^{64}$ hippocampal neurons infected with a lentiviral vector harboring Cre recombinase (Fig. 4d and Supplementary Fig. 4a).

IgSF8 immunohistochemistry of P28 mouse brain sections revealed strong immunoreactivity in CA3 SL and prominent labeling of several fiber tracts, such as corpus callosum (Supplementary Fig. 4b). As Igsf8 is strongly expressed in dorsal dentate GCs (https://hipposeq.janelia.org/), we crossed Igsf8 cKO mice with the dentate GC-specific Rbp4-Cre line (Supplementary Fig. 4a). This largely and selectively abolished IgSF8 immunoreactivity in SL (Fig. 4e), indicating that IgSF8 in the MF pathway is predominantly GC derived. Igsf $8 \mathrm{cKO}$ did not affect gross MF pathway morphology (Fig. 4e). To gain insight into the subcellular distribution of IgSF8, we first transfected primary cultured neurons. Surface HA-IgSF8 accumulated in distal growth cones (Supplementary Fig. 4c), where it colocalized with phosphorylated Ezrin-Radixin-Moesin (ERM) proteins (Supplementary Fig. 4d), which link cell-surface receptors and the actin cytoskeleton $^{65}$, and control neurite outgrowth and process formation 66,67 . We then performed in utero electroporation of embryonic day E15.5 mouse embryos to transfect single dentate GCs, with a plasmid expressing membrane GFP (mGFP) to label MF bouton and filopodia, and HA-tagged IgSF8 to visualize IgSF8 localization at MF synapses (Fig. 4f). HA-IgSF8 localized to both the main MF bouton, as well as to distal filopodia (Fig. $4 \mathrm{~g}$ and Supplementary Fig. 4e). These results indicate that GC-derived IgSF8 localizes to two key sites in the CA3 microcircuit: the MF bouton synapsing onto CA3 dendrites, and the filopodia synapsing onto SL interneurons.

Loss of IgSF8 impairs MF synapse architecture and filopodia density. To determine the consequences of dentate GC-specific loss of IgSF8 on MF synaptic structure, we first analyzed MF bouton ultrastructure in Igsf8 cKO mice. We prepared hippocampal sections from $\mathrm{P} 30 \mathrm{Rbp} 4-\mathrm{Cre}: \operatorname{Igs} f 8 \mathrm{cKO}$, and control littermates and imaged synapses by transmission electron microscopy (TEM; Fig. 5a). We observed a clear reduction in active zone (AZ) number and length, and a corresponding decrease in PSD number and length (Fig. 5b), indicating that loss 
a

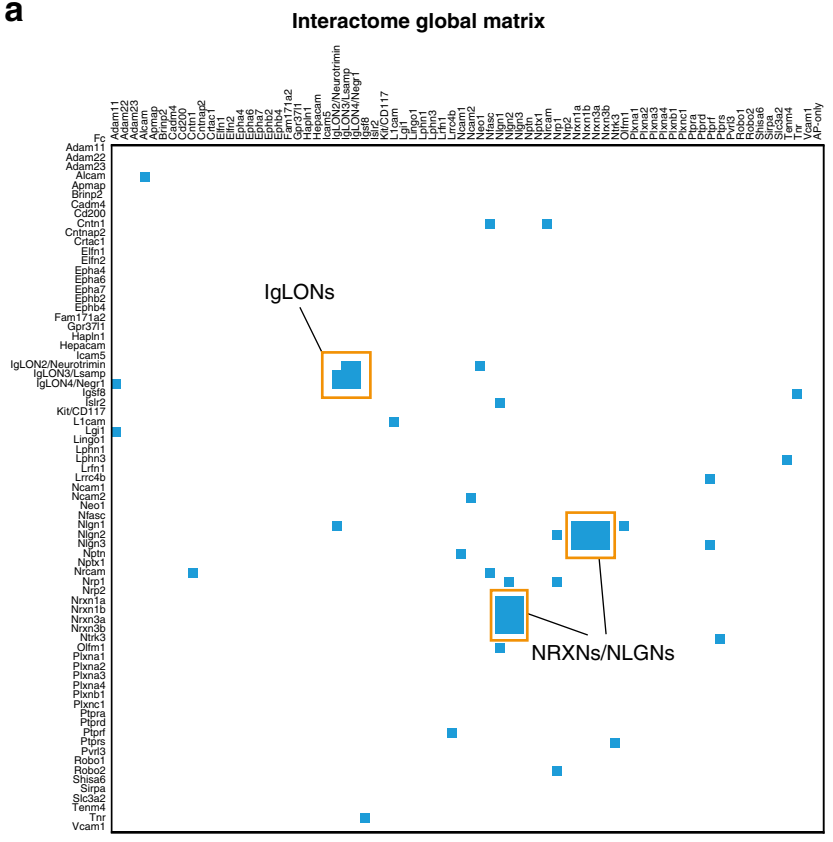

b

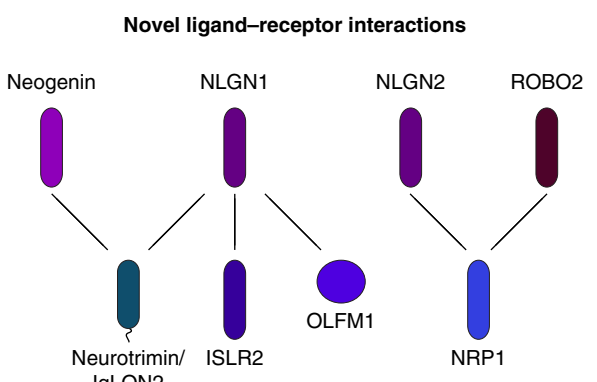

IgLON2

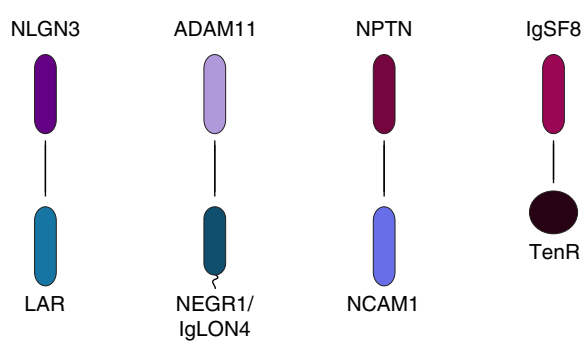

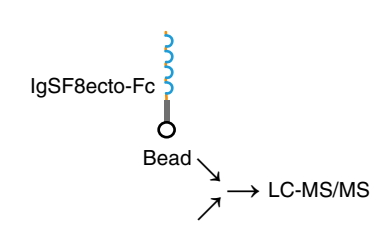

Whole brain synaptosome extracts

or

MF synaptosome extracts

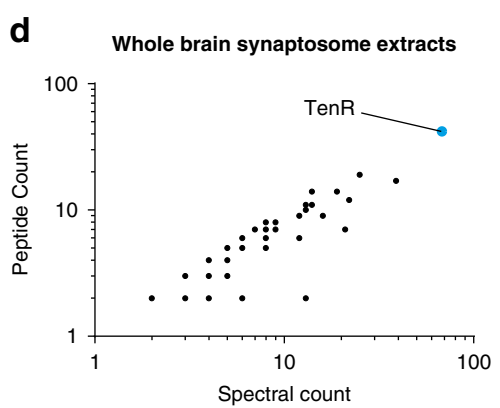

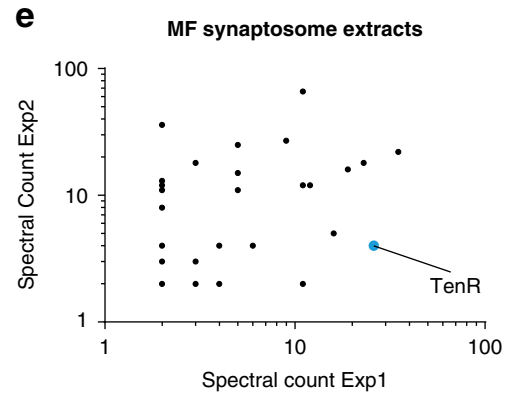

g

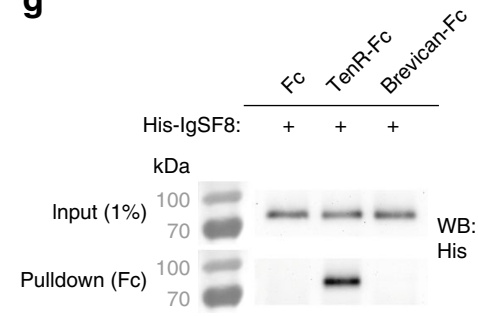

h

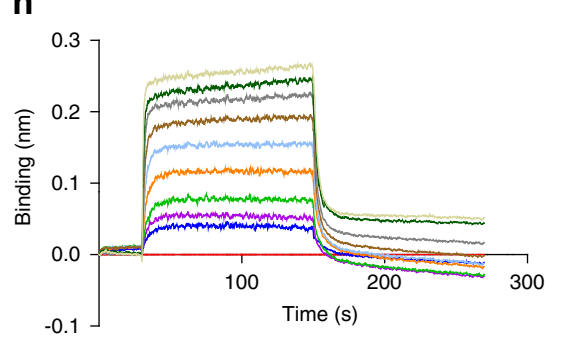
myc-LRRTM2

Nrxn1b-Fc
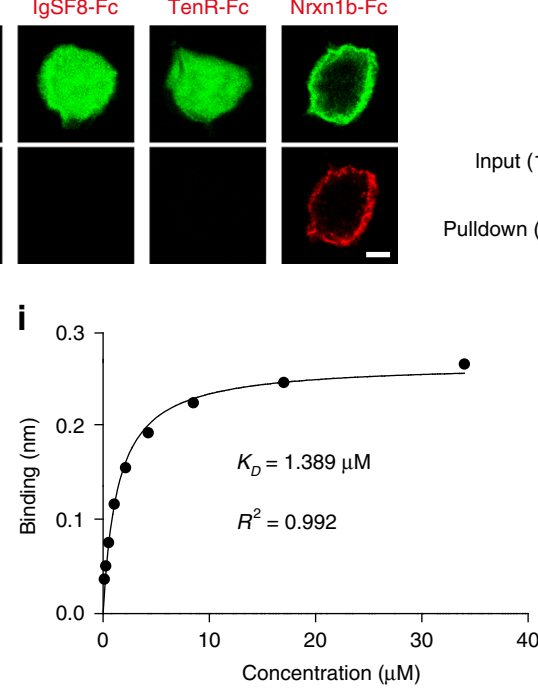

of IgSF8 impairs MF synaptic architecture. MF bouton number and area were not affected in Igsf8 cKO mice (Fig. 5c, d).

To analyze the morphology of MF bouton and filopodia, we injected adeno-associated viral (AAV) vectors expressing mGFP and Cre recombinase in $\mathrm{P} 7$ Igsf8 $\mathrm{cKO}$ mice to remove IgSF8 expression. AAV-mGFP was used as a control (Fig. 5e). We imaged P28 MF boutons using confocal microscopy and reconstructed them in $3 \mathrm{D}$. We observed a dramatic decrease in the number of filopodia emerging from the main MF bouton in Igsf8 cKO mice compared to controls (Fig. 5f, g). In addition, we observed a mild reduction in volume of the MF bouton (Fig. 5f, g). Together, these results show that loss of IgSF8 in GCs impairs MF synapse architecture and strongly reduces filopodia density. 
Fig. 3 MF synapse CSP interactome screening. a Global matrix of data obtained from three independent experiments for interactions between 73 AP- and Fc-tagged proteins in both orientations (i.e., AP-X vs Fc-Y and AP-Y vs Fc-X), resulting in $73 \times 73=5329$ experimental points and 2701 unique pairwise interactions. Columns contain AP-fusion baits, including the AP-only construct as negative control, whereas rows contain Fc-fusion preys. Positive ligand-receptor interactions are indicated in blue. Two major modules of known ligand-receptor pairs are highlighted in orange: the NRXNs-NLGNs and the IgLON family. $\mathbf{b}$ Interaction networks of novel ligand-receptor pairs identified. Among these, the interaction between IgSF8 and TenR was the only one including a CSP of uncharacterized brain function. c Cartoon illustrating the Ecto-Fc MS workflow to validate IgSF8-TenR interaction in synaptosomes. d Graph of spectral and peptide counts of proteins captured in a pull-down experiment using whole rat brain synaptosome lysates. Only proteins which were absent in Fc controls and present with $\geq 2$ spectral counts per IgSF8-ecto-Fc experiment are included. TenR is highlighted in blue. e Graph of spectral counts of proteins captured in two independent pull-down experiments using P21 mouse MF synaptosome lysates. Only proteins with $\leq 1$ spectral counts in Fc controls and $\geq 2$ spectral counts per IgSF8-ecto-Fc experiment are included. TenR is highlighted in blue. $\mathbf{f}$ Confocal images of cell-surface binding assays in transfected HEK293T cells show IgSF8-TenR interaction. Negative and positive controls used in the cell-surface binding assays are also shown. $\mathbf{g}$ Direct binding assays show direct and specific interaction of IgSF8 with TenR, but not with ECM protein Brevican. $\mathbf{h}$ Biolayer interferometry experiment of the IgSF8-TenR interaction. A concentration range of purified IgSF8 (34-0.133 $\mu \mathrm{M})$ was used in twofold dilution plus buffer alone (red straight line). i Plotting the binding response $(\mathrm{nm})$ vs IgSF8 concentration provided a measure of the affinity of the interaction $\left(K_{\mathrm{D}} \sim 1.4 \mu M\right)$. Source data are provided as a Source data file. Scale bar in $\mathbf{f} 5 \mu \mathrm{m}$.

Loss of IgSF8 impairs spontaneous synaptic transmission in CA3 neurons. To analyze the functional consequences of IgSF8 removal in GCs on transmission at the MF synapse, we first performed whole-cell voltage-clamp recordings of CA3 pyramidal neurons in acute hippocampal slices from P27-35 Rbp4-Cre:Igsf8 $\mathrm{cKO}$ and control littermates. The frequency of spontaneous excitatory postsynaptic currents (sEPSCs) was strongly reduced in Igsf $8 \mathrm{cKO}$ mice compared to controls (Fig. 6a-c), consistent with the decrease in the number of synaptic junctions at Igsf $8 \mathrm{cKO} \mathrm{MF}$ boutons (Fig. 5b). The amplitude of sEPSCs was also reduced in Igsf8 cKO (Fig. 6d, e), consistent with the reduced length of synaptic junctions (Fig. 5b). Decay time of sEPSCs was not affected (Supplementary Fig. 5a). Histogram analysis showed a loss of large-amplitude sEPSCs in Igsf8 cKO mice (Supplementary Fig. $5 \mathrm{~b}, \mathrm{c})$ that originate from MF synapses ${ }^{68}$, supporting an impairment of MF-CA3 synapses in the absence of GC-derived IgSF8.

To specifically assess synaptic transmission at MF-CA3 synapses, we injected AAV vectors to express Cre-dependent Channelrhodopsin-2 (DIO-ChR2) in dentate GCs of Rbp4-Cre: Igsf $8 \mathrm{cKO}$ and control littermates, and optically stimulated $\mathrm{MF}$ axons while performing whole-cell voltage-clamp recordings from CA3 pyramidal neurons (Fig. 6f). This approach enables us to selectively stimulate MFs lacking IgSF8 in Igsf $8 \mathrm{cKO}$ mice. We analyzed paired-pulse facilitation, a form of short-term plasticity, to assess MF-CA3 presynaptic properties, but found no differences in the amplitude of the first evoked EPSC or the paired-pulse ratio between Igsf $8 \mathrm{cKO}$ and control mice (Fig. 6g-i). Thus, loss of presynaptic IgSF8 impairs spontaneous synaptic transmission, but does not alter evoked transmission at MF-CA3 synapses.

Reduced feedforward inhibition and increased excitability of CA3 neurons in Igsf8 cKO. We next assessed the functional consequences of conditional removal of IgSF8 on feedforward inhibition in the CA3 microcircuit. Given the robust decrease in the number of MF filopodia in Igsf8 cKO mice (Fig. $5 \mathrm{~g}$ ), we hypothesized that stimulation of Igsf $8 \mathrm{cKO}$ MFs would result in reduced recruitment of feedforward inhibition. To test this, we again expressed DIO-ChR2 in dentate GCs of Rbp4-Cre:Igsf8 $\mathrm{cKO}$ and control littermates to optically stimulate MF axons, while performing whole-cell voltage-clamp recordings from CA3 pyramidal neurons (Fig. 7a, b). Light-evoked EPSCs and IPSCs were recorded from the same CA3 pyramidal neuron. We observed a strong reduction in eIPSC amplitude (Fig. 7c) and a robust increase in the eEPSC/eIPSC ratio in Igsf $8 \mathrm{cKO}$ compared to littermate controls (Fig. 7d). Thus, loss of IgSF8 reduces feedforward inhibition in the CA3 microcircuit, resulting in excitation-inhibition imbalance of CA3 pyramidal neurons.

Feedforward inhibition mediated by MF filopodia plays an important role in the control of CA3 pyramidal neuron excitability ${ }^{13}$. To determine the consequences of reduced feedforward inhibition in Igsf8 cKO mice on CA3 pyramidal neuron excitability, we optically stimulated MFs and analyzed action potential firing in CA3 pyramidal neurons in Rbp4-Cre: Igsf $8 \mathrm{cKO}$ and control littermates (Fig. 7e). In this experiment, we first recorded CA3 pyramidal neurons in voltage-clamp mode at $-82 \mathrm{mV}$ (the $\mathrm{Cl}^{-}$reversal potential) and stimulated MFs to establish a comparable eEPSC amplitude of $\pm 400 \mathrm{pA}$ in both conditions (Fig. 7f). CA3 neurons were then switched to currentclamp mode to record resting membrane potential, which was comparable between both groups (Fig. 7f), followed by optical stimulation of MFs with a $10 \mathrm{~Hz}$ train of 20 stimuli. We found that CA3 neurons in Igsf8 cKO mice started firing action potentials earlier during the train than in control littermates (Fig. 7g), supporting the notion that reduced feedforward inhibition in the absence of IgSF8 leads to increased MFinduced excitability of CA3 neurons. Taken together, these results show that loss of IgSF8 reduces feedforward inhibition resulting in increased excitability of CA3 pyramidal neurons, indicating that IgSF8 is an organizer of hippocampal CA3 microcircuit connectivity and function.

\section{Discussion}

Precise control of the dynamic interaction between excitatory and inhibitory neurons connected into microcircuits is critical for circuit output and cognitive function. Here, we surveyed the proteome of isolated MF synaptosomes to identify novel regulators of synaptic connectivity in the CA3 microcircuit. Our results uncover a diverse CSP repertoire at MF synapses and reveal the uncharacterized neuronal receptor IgSF8, as a critical regulator of CA3 microcircuit connectivity and function.

Characterization of the MF synapse CSP landscape. Dissecting the molecular composition of specific connections remains a major challenge. Sorting of growth cones ${ }^{69}$ and specific synapse types (Pfeffer et al. ${ }^{70}$, this study), imaging-based approaches ${ }^{71-73}$, affinity purification $^{74}$, and proximity biotinylation ${ }^{75-77}$ each have their advantages and disadvantages ${ }^{78}$. Here, we combined biochemical enrichment, antibody labeling of a synapse type-enriched surface marker, and fluorescent sorting to isolate MF synapses. When coupled with highly sensitive MS analysis, dissecting the proteome of a specific type of synapse with well-characterized connectivity, 
a

Topology of IgSF8

$\mathrm{N}$-terminal

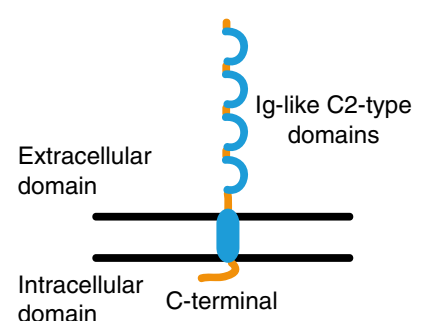

b

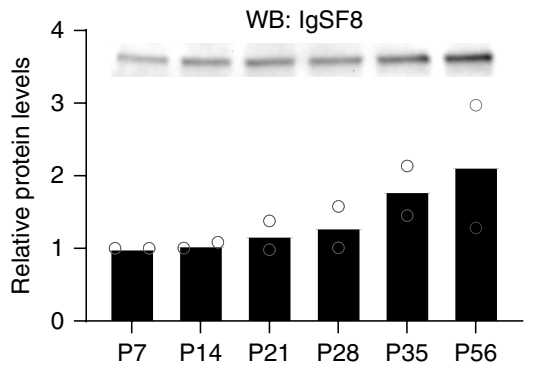

C

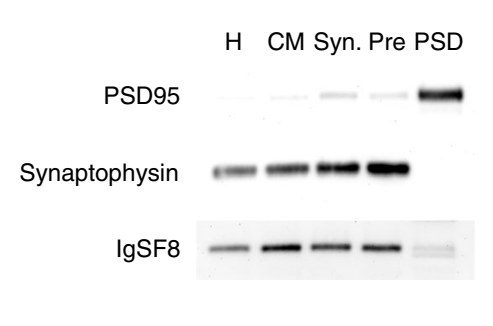

d

Igsf8 cKO E18 Primary hippocampal cultures

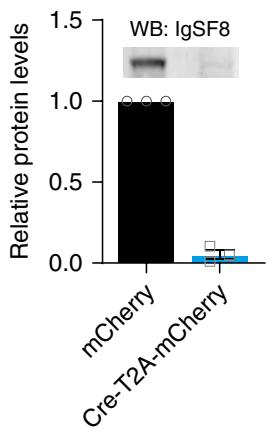

f

IUE at E15.5 WT $\rightarrow$ P28 analysis

FUGW-mGFP-T2A-HA-IgSF8

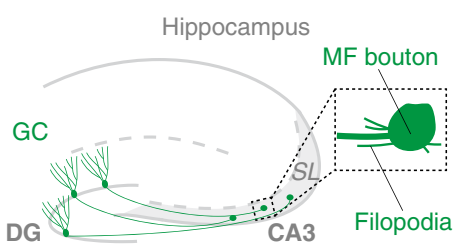

e
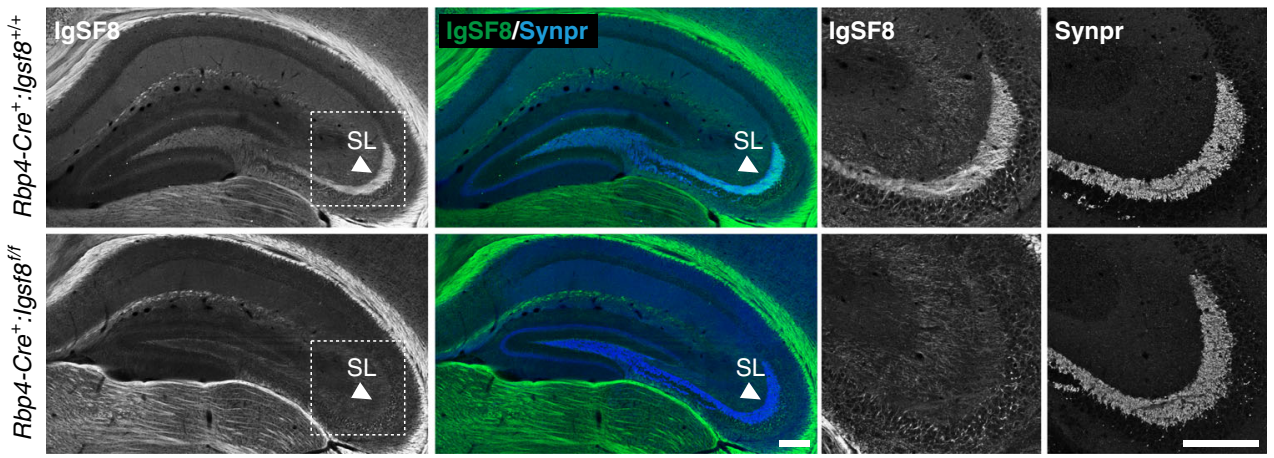

Fig. 4 IgSF8 localizes to MF boutons and filopodia. a Cartoon of IgSF8 protein topology. b Western blot analysis of lgSF8 protein expression levels in mouse hippocampal homogenates at different developmental time points. Bar graph shows mean. Quantification from two independent experiments. c Subcellular fractionation using whole rat brains. H homogenate, CM crude membrane, Syn synaptosomes, Pre presynaptic fraction, PSD postsynaptic density fraction. d Western blot analysis of Igsf8 cKO mouse primary hippocampal cultures infected with a lentiviral vector harboring Cre recombinase or control vector. Bar graph shows mean \pm SEM. Quantification from three independent experiments. e Confocal images of P28 Rbp4-Cre:lgsf8 cKO mouse hippocampal sections immunostained for IgSF8 and Synpr. Arrowheads indicate SL. Magnified insets of the SL in CA3 are shown on the right. $\mathbf{f}$ In utero electroporation setup to sparsely label DG granule cell neurons using membrane GFP (mGFP) and analyze the localization of HA-IgSF8 in MF synapses. g Stack of confocal images showing a mGFP-labeled MF bouton and respective filopodia (in blue in the merge). HA-lgSF8 is concentrated in the MF bouton, but also observed in defined regions along the filopodia, including their terminals (in green in the merge). More examples are shown in Supplementary Fig. 4e. Source data are provided as a Source data file. Scale bars in e $200 \mu \mathrm{m}$ and in $\mathbf{g} 10 \mu \mathrm{m}$.

morphology, and functional properties, offers the advantage that its molecular composition can be linked to its structural and functional features, at the single-synapse level. Using this approach, we provide the first insight into the CSP landscape and cell-surface interactome of a specific excitatory synapse type.

We identified and validated a rich CSP repertoire at MF synapses. The large majority of the CSPs we identify has not previously been shown to localize or function at MF synapses, and approximately half do not have a synaptic function. Four CSPs (APMAP, FAM171A2, BRINP2, and IgSF8) lack a known function in the brain altogether. Together, our findings underscore the complexity of the MF synaptic proteome and illustrate the advantage of synapse type-specific proteomics in uncovering uncharacterized synaptic proteins.
Our approach identified several CSPs at the MF synapse of uncharacterized function in the brain. The transmembrane protein APMAP interacts with the amyloid precursor protein (APP) and the $\gamma$-secretase complex and negatively regulates amyloid- $\beta$ production ${ }^{79,80}$. The transmembrane protein FAM171A2 induces membrane protrusions in cultured cells upon overexpression and is required for invasive growth of melanoma cells ${ }^{81}$. Immunohistochemistry validated the presence of FAM171A2 protein in the MF pathway, but its role there remains unknown. The secreted glycoprotein BRINP2 is highly expressed in CA3 (ref. ${ }^{82}$ ) and validated to be present in MF synaptosomes. Brinp2 $\mathrm{KO}$ mice display hyperactivity ${ }^{83}$, but the function of BRINP2 is not known. Finally, the transmembrane protein IgSF8 interacts with tetraspanins and integrins ${ }^{84-86}$, and is thought to modulate cell motility 
a

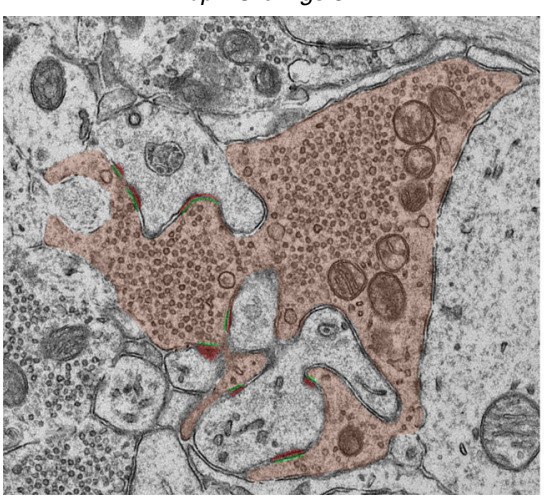

C

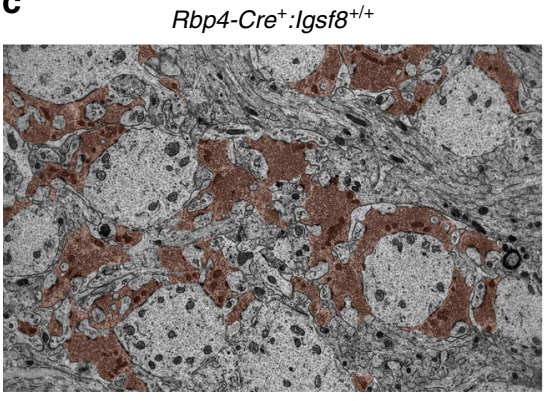

e
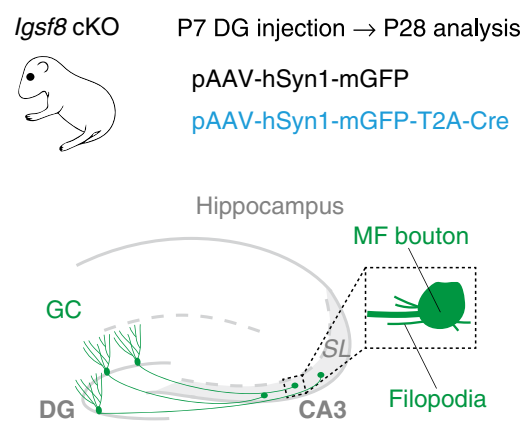

Rbp4-Cre $:$ Igsf8 ${ }^{f / f}$

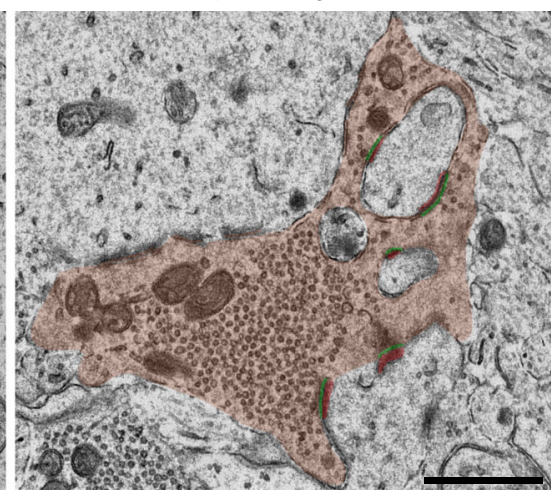

Rbp4-Cre $:$ Igsf8 ${ }^{f / f}$

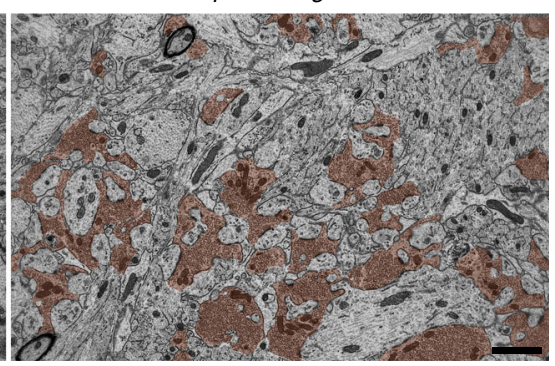

f

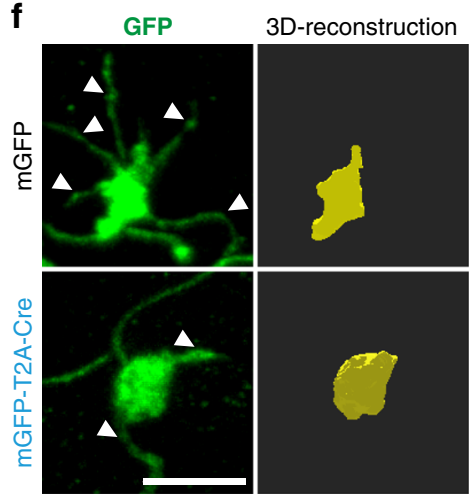

b
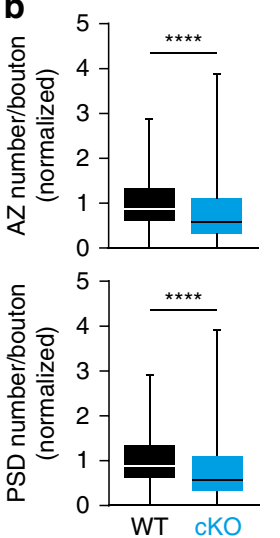

d
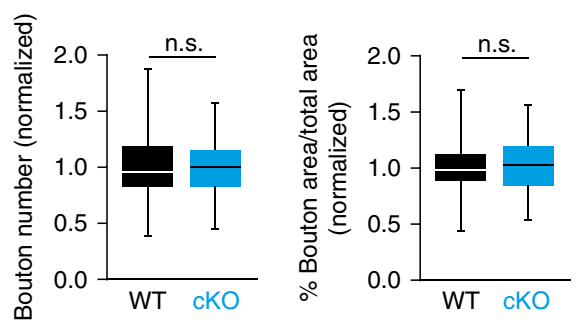

g

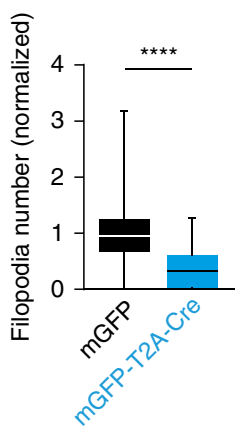

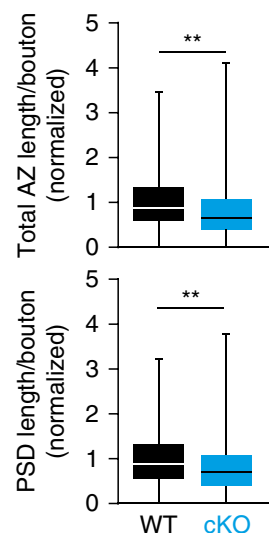

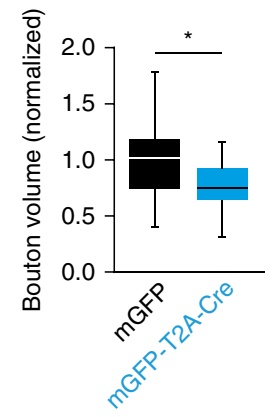

Fig. 5 Loss of IgSF8 impairs MF synapse architecture and filopodia density. a Electron microscope images of MF-CA3 synapses from Rbp4-Cre:Igsf8 cKO and WT littermates (5000× magnification). MF boutons are highlighted in orange. AZs and PSDs are highlighted in green and red, respectively. $\mathbf{b}$ Graphs show quantification of analysis done in $\mathbf{a}$ in littermate mice examined over three independent experiments (WT, $n=135$ boutons and $c K O, n=136$ ). ${ }^{\star \star \star \star *} P<0.0001$ and ${ }^{* \star} P=0.0028$ in upper panel. ${ }^{\star \star \star \star} P<0.0001$ and ${ }^{*} P=0.0056$ in lower panel. c Electron microscope images of MF-CA3 synapses from Rbp4-Cre:Igsf8 cKO and WT littermates (2500× magnification) to analyze number and area of MF boutons, highlighted in orange. d Graphs show quantification of analysis done in $\mathbf{c}$ in littermate mice examined over three independent experiments (WT, $n=103$ images and cKO, $n=93$ ). e Experimental design to analyze structural changes in MF synapses following deletion of Igsf8 specifically in DG granule cells. $\mathbf{f}$ Stacks of confocal images of individual MF boutons (left) and respective 3D reconstructions (right) to analyze number of MF bouton filopodia and MF bouton volume. Arrowheads show filopodia emerging from MF boutons. $\mathbf{g}$ Graphs show quantification of analysis done in $\mathbf{f}$ in littermate mice examined over three independent experiments (mGFP, $n=27$ boutons and mGFP-T2A-Cre $n=29$ ). ${ }^{\star \star \star \star} P<0.0001$ and ${ }^{\star} P=0.0149$ in $\mathbf{g}$. Box-and-whisker plots in $\mathbf{b}$, $\mathbf{d}$ and $\mathbf{g}$ show median, interquartile range, minimum, and maximum. Two-sided Mann-Whitney tests were used in $\mathbf{b}$ and $\mathbf{g}$. Unpaired Student's $t$ tests were used in d. n.s. not significant. Source data are provided as a Source data file. Scale bars in a $1 \mu \mathrm{m}$, in c $2 \mu \mathrm{m}$, and in $\mathbf{f} 5 \mu \mathrm{m}$.

in immune and cancer cells through tetraspanin microdomains ${ }^{87,88}$. Interestingly, both FAM171A2 and IgSF8 are involved in membrane protrusion and motility, which might be relevant for structural plasticity of MF synapses ${ }^{89}$.

Multiple secreted and ECM proteins were validated at MF synapses (BRINP2, CRTAC1, NPTX1, OLFM1, and TenR). The synaptic function of CRTAC1, which acts as a Nogo receptor-1 antagonist in regulating lateral olfactory axon tract bundling in early development ${ }^{90}$, is not known. Many CSPs with roles in the early stages of circuit assembly (NRP1, NRP2, CRTAC1, ISLR2,
Neogenin, ROBO2, PlexinA1, and PlexinA3) were validated at mature MF synapses. With the exception of the NRPs ${ }^{91-93}$, their synaptic role remains unknown. Our findings suggest that secreted/ECM proteins and axon guidance-related proteins shape MF synaptic connectivity and continue to play a role at mature MF synapses.

Several CSPs with an established role in MF synapse development and function, including the postsynaptic receptors EphB2 (ref. ${ }^{94}$ ) and EphA4 (ref. ${ }^{95}$ ), and the presynaptic adhesion molecule NRXN1 (ref. ${ }^{96}$ ), were identified (see Supplementary Data 2 for a 

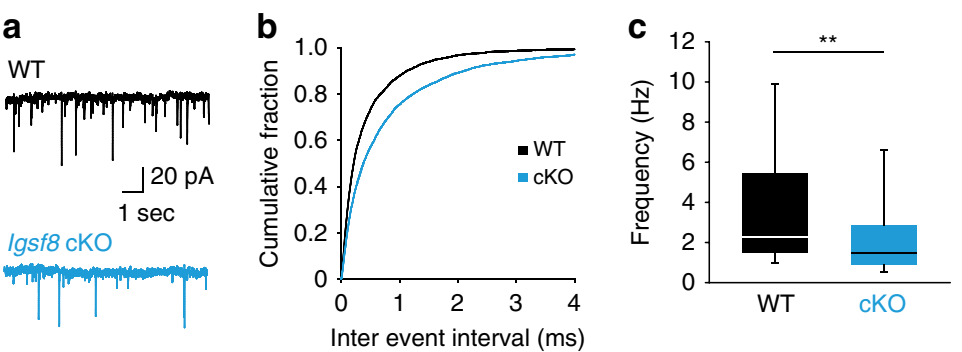

f

Rbp4-Cre :Igsf8 cKO

g<smiles>C1=CC2CCCCC2C1</smiles>

P7 DG injection $\rightarrow$ P27-35 analysis pAAV-Ef1a-DIO-ChR2-EYFP

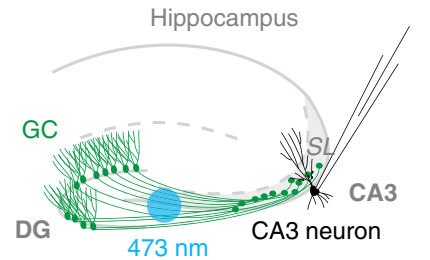

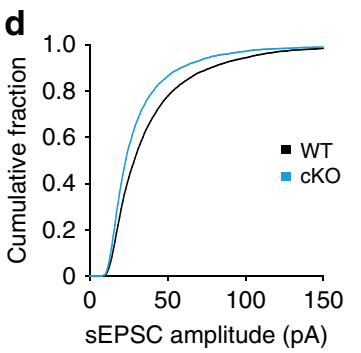

h

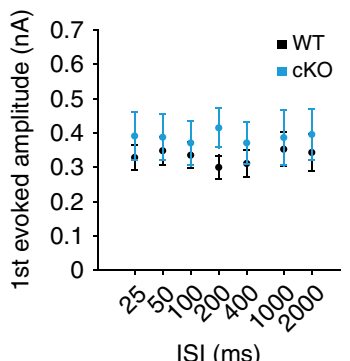

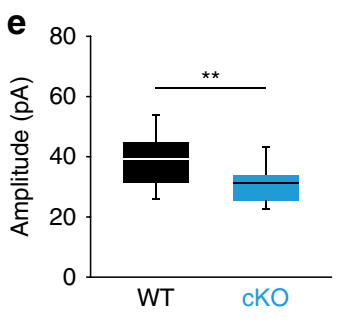

i

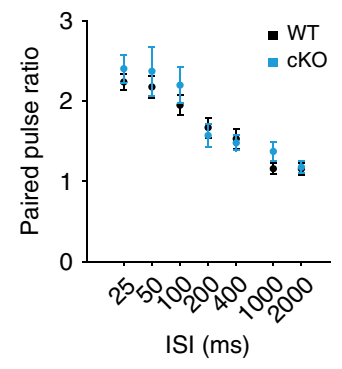

Fig. 6 Loss of IgSF8 impairs spontaneous synaptic transmission in CA3 neurons. a Representative sEPSC traces from whole-cell voltage-clamp recordings of CA3 neurons in acute hippocampal slices of Rbp4-Cre:Igsf8 cKO and WT littermates. $\mathbf{b}$ Cumulative distribution of sEPSC inter-event intervals. c Quantification of sEPSC frequency in littermate mice examined over three independent experiments $(\mathrm{WT}, n=31$ neurons and cKO, $n=38)$. ${ }^{\star \star} P=0.008$. d Cumulative distribution of sEPSC amplitudes. e Quantification of sEPSC amplitudes in littermate mice examined over three independent experiments (WT, $n=31$ neurons and $\mathrm{CKO}, n=38$ ). ${ }^{\star \star} P=0.002$. $\mathbf{f}$ Cartoon illustrating whole-cell voltage-clamp recordings of CA3 neurons to measure MF-evoked responses in acute hippocampal slices of Rbp4-Cre:Igsf8 cKO and WT littermates using optogenetics. $\mathbf{g}$ Representative traces of paired-pulse ratio in Rbp4Cre:Igsf8 cKO and WT littermates using optogenetics. $\mathbf{h}$ Quantification of first evoked amplitudes in littermate mice examined over three independent experiments (WT, $n=23$ neurons and $c K O, n=21$ ). $\mathbf{i}$ Quantification of paired-pulse ratios in littermate mice examined over three independent experiments (WT, $n=23$ neurons and $c K O, n=21$ ). Box-and-whisker plots in $\mathbf{c}$ and e show median, interquartile range, minimum, and maximum. Graphs in $\mathbf{h}$ and $\mathbf{i}$ show mean \pm SEM. Two-sided Mann-Whitney tests were used in $\mathbf{c}$ and $\mathbf{e}$. Source data are provided as a Source data file.

complete overview). In addition, the secreted glycoprotein C1QL3 (ref. ${ }^{97}$ ), the classic type II cadherin Cadherin-9 (ref. ${ }^{36}$ ), and the G-protein-coupled receptor-like protein GPR158 (ref. ${ }^{27}$ ) were also detected in sorted MF synaptosomes (Supplementary Data 1). Interestingly, the loss of function phenotypes of these proteins display similarities, but are rarely identical to one another. Together, these findings suggest that a combinatorial code of CSPs that act in a partially redundant manner defines MF synapse identity. The CSP diversity at the MF synapse may also reflect heterogeneity within this synaptic population. The maturational state of MF synapses varies due to the continuous integration of newborn GCs into the hippocampal circuit ${ }^{98}$. Different histories of synaptic activity may also diversify CSP composition.

Characterization of the MF synapse cell-surface interactome. We used automated interactome screening to map ligand-receptor relationships among the MF synapse CSPs we identified, characterizing a synapse type-specific network of CSP modules and uncovering ten interactions that have not been reported previously. Nine of these remain to be validated by independent methods, but several are worth highlighting here. The secreted glycoprotein OLFM1 interacts with APP 99 and AMPARs ${ }^{100-103}$, and regulates the mobility of AMPARs and short-term plasticity in cultured neurons ${ }^{104}$. The OLFM1-NLGN1 interaction identified here suggests that OLFM1 is well positioned to modulate excitatory neurotransmission and postsynaptic plasticity through different protein complexes. We further identified several ligand-receptor modules featuring IgLON family members, including the interaction of
Neurotrimin/IgLON2 with NLGN1 and of NEGR1/IgLON4, with the adhesion molecule ADAM11. IgLONs are synaptic adhesion molecules that span the synaptic cleft ${ }^{56}$. NEGR1/IgLON4 regulates synapse number in cultured hippocampal neurons ${ }^{105}$. Future studies addressing the synaptic functions of IgLONs should take the putative binding partners identified here into consideration. Finally, we identified several interactions, including those of NLGN1-ISLR2, NLGN2-NRP1, and Neurotrimin/IgLON2-Neogenin, that suggest considerable cross talk between synaptic adhesion molecules and axon guidance-related receptors.

As the MF synapse cell-surface interactome screen was limited to those CSPs that reached significance in our proteome analysis, tests only binary interactions, does not take splice variants into account, and does not detect binding affinities weaker than $\pm 10 \mu \mathrm{M}$ (refs. ${ }^{25,26,53,56}$ ), the MF synapse CSP interactome will likely be even more complex. Synapse type-specific proteome analysis and cell-surface interactome analysis will be used in future studies to determine to what extent developmental stage, and neural activity influence cell-surface composition and interactome of MF synapses.

IgSF8 regulates CA3 microcircuit connectivity and function. Synapse type-specific proteome profiling and CSP interactome screening uncovered IgSF8 as an uncharacterized neuronal receptor for the ECM protein TenR at the MF synapse. A previous immunohistochemical study demonstrated transient IgSF8 immunoreactivity in olfactory sensory neuron axon terminals during synaptogenesis ${ }^{106}$. Lesion-induced reformation of 
a
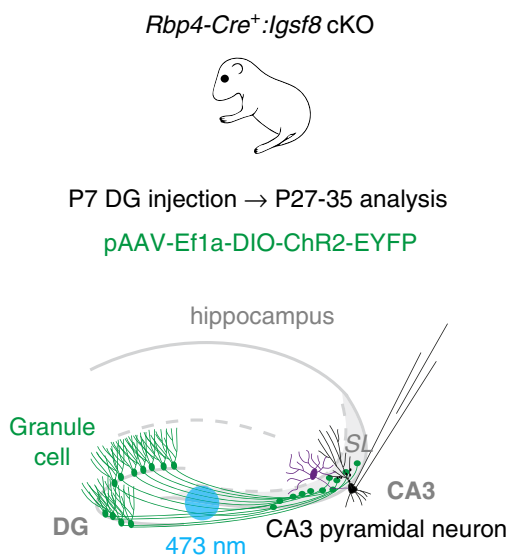

e

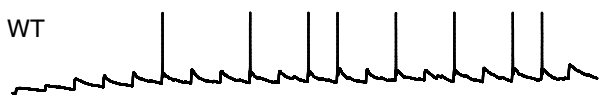

$\longrightarrow 20 \mathrm{mV}$

Igsf8 cKO

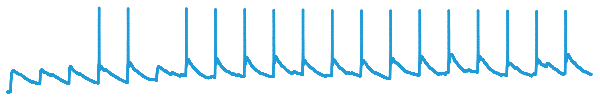

b

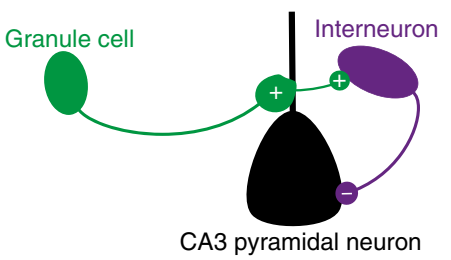

d

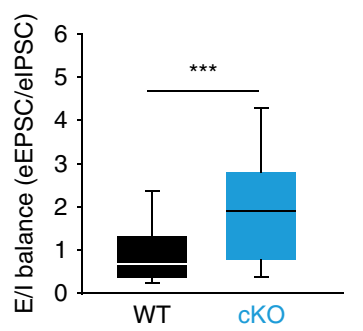

$\mathbf{f}$

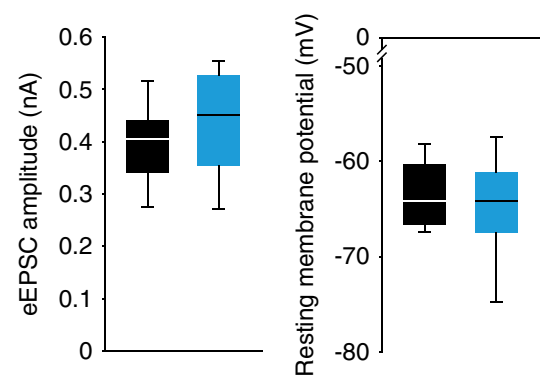

C

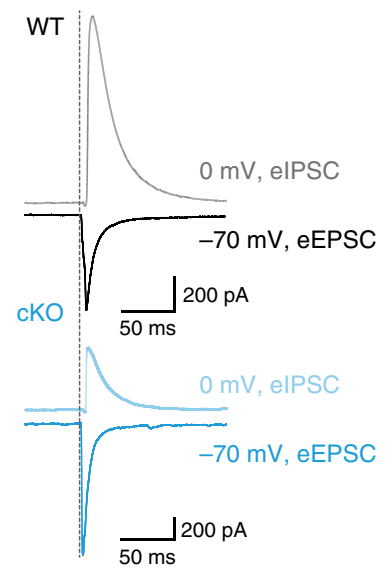

$9-W T$

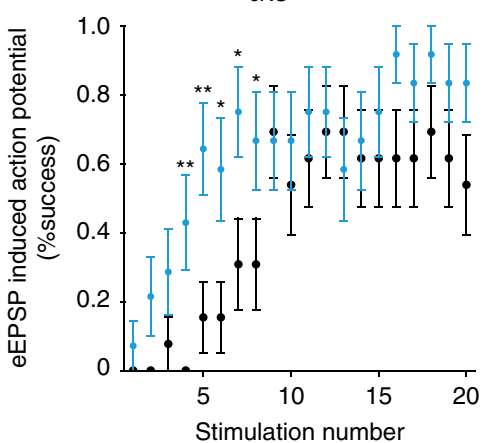

Fig. 7 Reduced feedforward inhibition and increased excitability of CA3 neurons in Igsf8 cKO. a Cartoon illustrating whole-cell voltage-clamp recordings of CA3 neurons to measure MF-evoked responses in acute hippocampal slices of Rbp4-Cre:Igsf8 cKO and WT littermates using optogenetics. b Cartoon illustrating feedforward inhibition microcircuit in CA3. Plus and minus signs represent excitatory and inhibitory synapses, respectively. c Representative eEPSC and eIPSC traces from CA3 pyramidal neurons in WT or cKO mice. $\mathbf{d}$ Quantification of excitation-inhibition balance in CA3 neurons in WT and cKO mice in littermate mice examined over three independent experiments (WT, $n=35$ neurons and $c K O, n=29$ ). ${ }^{\star \star \star} P=0.0003$. e Representative eEPSP traces from CA3 pyramidal neurons in WT or CKO mice. $\mathbf{f}$ Quantification of resting membrane potentials and eEPSC amplitudes of CA3 neurons in WT and cKO littermate mice examined over three independent experiments (WT, $n=13$ neurons and cKO, $n=14$ ). $\mathbf{g}$ Quantification of induced action potential firing in CA3 neurons in WT and cKO littermate mice examined over three independent experiments (WT, $n=13$ neurons and cKO, $n=14)$ after a $10 \mathrm{~Hz}$ train of 20 stimuli. ${ }^{\star} P=0.03$ and ${ }^{\star} P=0.01$ in $\mathbf{g}$. Box-and-whisker plots in $\mathbf{d}$ and $\mathbf{f}$ show median, interquartile range, minimum, and maximum. Graph in $\mathbf{g}$ shows mean \pm SEM. Two-sided Mann-Whitney tests were used in $\mathbf{d}$ and $\mathbf{g}$. Source data are provided as a Source data file.

synapses caused IgSF8 to reappear in terminals, whereas blocking olfactory activity prevented the disappearance of IgSF8 from mature synapses ${ }^{106}$. Similarly, the EWI-subfamily member IgSF3 transiently localizes to developing cerebellar GC axon terminals ${ }^{107}$. The localization of IgSF8 to the MF bouton and filopodia suggested a functional requirement for IgSF8 at both sites. Conditional deletion of Igsf8 in dentate GCs reduced length, and number of AZs and PSDs in the main MF bouton, suggesting that IgSF8 regulates synaptic junction architecture and number. This defect is reminiscent of the reduction in $\mathrm{AZ}$ length and number at perisomatic inhibitory synapses in CA1 pyramidal neurons in Tnr KO mice ${ }^{62}$. Together, these observations suggest that IgSF8 regulates synapse number and length, possibly via an interaction with TenR, which localizes to the excitatory synaptic cleft of cultured neurons ${ }^{108}$.

In addition to defects in the main MF bouton, conditional deletion of Igsf8 in dentate GCs strongly decreased the number of MF filopodia that synapse onto SL interneurons ${ }^{9}$. The cytoplasmic tail of IgSF8 binds ERM proteins ${ }^{109}$ and alpha-actinin ${ }^{110}$, both of which are linked to filopodia formation. In cultured neurons, we observed that cell-surface IgSF8 colocalized with phosphorylated (activated) ERM proteins. Activation of
ERM proteins in growth cone filopodia is required for axon growth $^{111,112}$. Treating cultured chick tectal neurons with TenR induces the formation of actin-rich filopodia ${ }^{113}$. Together, these observations suggest that IgSF8, via an interaction with TenR, may promote the formation and/or stabilization of MF filopodia.

MF filopodia mediate feedforward inhibition of CA3 neurons, which plays a key role in CA3 microcircuit function in normal and pathological conditions ${ }^{12,14-16}$. Interestingly, TenR immunoreactivity is highly concentrated around parvalbumin-positive SL interneurons ${ }^{61,63}$, suggesting that the interaction of IgSF8 and TenR may control filopodia-SL interneuron connectivity, and play an important role in controlling dynamic interactions between excitatory and inhibitory neurons in the CA3 microcircuit that are important for memory. Although speculative, the localization of IgSF8 to both the main MF bouton and filopodia might constitute a molecular mechanism to coordinate changes at both types of synapses in the CA3 microcircuit in response to changes in activity.

In conclusion, using proteomic profiling of a specific synapse in the CA3 microcircuit, we identified multiple uncharacterized synaptic CSPs and uncovered IgSF8 as a regulator of connectivity within this circuit. Although our study focused on the cell-surface 
proteome of the MF synapse, our dataset can be used to analyze other classes of proteins important for synaptic function and may be of use in efforts to model synaptic connectivity in the DG ${ }^{114}$. A combination of approaches, including the synapse type-specific proteomic profiling approach we apply here, will ultimately be required to elucidate the molecular mechanisms underlying the highly precise patterns of connectivity that are required for proper circuit function.

\section{Methods}

Animals. All animal experiments were conducted according to the KU Leuven ethical guidelines and approved by the KU Leuven Ethical Committee for Animal Experimentation (approved protocol numbers ECD P037/2016, P014/2017, and P062/2017). Mice were maintained in a specific pathogen-free facility under standard housing conditions with continuous access to food and water. Mice used in the study were 1-8-weeks old and were maintained on $14 \mathrm{~h}$ light, $10 \mathrm{~h}$ dark light cycle from 7 to $21 \mathrm{~h}$. Wild-type (WT) C57BL/6J mice were obtained from JAX. The Igsf $8 \mathrm{cKO}$ mouse line was obtained from the Riken Institute ${ }^{64}$, while the Rpb4-Cre line was obtained from GENSAT. Genotypes were regularly checked by PCR analysis. For euthanasia, animals were either anesthetized with isoflurane, and decapitated or injected with an irreversible dose of ketamine-xylazine.

Neuronal cultures. Hippocampal neurons were cultured from E18 Igsf8 cKO mice Neurons were plated on poly-D-lysine (Millipore) and laminin (Invitrogen)-coated glass coverslips (Glaswarenfabrik Karl Hecht). Neurons were maintained in Neurobasal medium (Invitrogen) supplemented with B27, glucose, glutamax, penicillin/ streptomycin (Invitrogen), $25 \mu \mathrm{m} \beta$-mercaptoethanol (Sigma-Aldrich), and insulin (Sigma-Aldrich). To knock out Igsf8, neurons were infected with low-titer lentivirus expressing mCherry or mCherry-T2A-Cre 2 days after plating and collected at day in vitro 10 (DIV10).

Cortical neurons from E18 WT mice were cultured and maintained over an astroglial feeder layer in $60-\mathrm{mm}$ culture dishes containing Neurobasal medium. Neurons grew face down over the feeder layer, but were kept separate from the glia by wax dots on the neuronal side of the coverslips. To prevent overgrowth of glia, neuron cultures were treated with $10 \mu \mathrm{M}$ 5-Fluoro-2'-deoxyuridine (SigmaAldrich) at DIV3. Cultures were maintained in a humidified incubator of $5 \%$ ( vol $/ \mathrm{vol}) \mathrm{CO}_{2} / 95 \%(\mathrm{vol} / \mathrm{vol})$ air at $37^{\circ} \mathrm{C}$, feeding the cells once per week by replacing one-third of the medium in the dish ${ }^{115}$. Neurons were electroporated just before plating using an AMAXA Nucleofector kit (Lonza, Basel, Switzerland) or transfected with calcium phosphate at DIV1 with plasmid FUGW-GFP-T2A-HAIgSF8. At DIV7/8, neurons were fixed and immunostained using the primary antibodies rat anti-HA (1:500; Roche) and rabbit anti-pERM (1:1000; Cell Signaling) with standard procedures. To stain the pool of HA-IgSF8 at the surface, primary antibody anti-rat HA was incubated after fixing neurons, but before the permeabilization step. Anti-pERM primary antibody was incubated after neurons were permeabilized.

Cell Lines. HEK293T-17 human embryonic kidney cells (available source material information: fetus) were obtained from American Type Culture Collection (ATCC) cat\# CRL-11268. HEK293T-17 cells were grown in Dulbecco's modified Eagle's medium (DMEM; Invitrogen) supplemented with $10 \%$ fetal bovine serum (FBS Invitrogen) and penicillin/streptomycin (Invitrogen).

Plasmids. For the ELISA-based interactome assay, we used recombinant AP- or Fc-tagged constructs containing human or mouse extracellular domain (ectodomain) protein sequences derived from the appropriate entry in the UNIPROT database (Uniprot.org). Cloning boundaries and cloning strategy can be found elsewhere ${ }^{53,56}$. Briefly, DNA sequences encoding the ectodomains of transmembrane and GPI-anchored CSPs, or the entire protein sequence in the case of secreted proteins, lacking the signal peptide, were cloned in frame using the $5^{\prime}$ NotI and $3^{\prime}$ XbaI restriction sites of a modified pCMV6-XL4 expression vector. Synthesis of DNA sequences and cloning into pCMV6-XL4 were outsourced. Fc-fusion proteins contain a leader peptide (PLP, prolactin leader peptide) followed by a $\mathrm{N}$ terminal FLAG tag, ectodomain of interest, a 3 CPro cleavage site, and the dimeric human Fc domain. Similarly, AP-fusion proteins contain a leader peptide, a FLAG tag and ectodomain of interest. C-terminal to the ectodomain there is a human AP followed by a $\mathrm{Hi}_{6}$ tag. For cloning NRXN ectodomains, we used DNA sequences of rat NRXN1 $\alpha-(-S 4)$, mouse NRXN1 $\beta-(-S 4)$, mouse NRXN3 $\alpha-(-S 4)$ (cDNA gift from Ann Marie Craig's lab), and rat NRXN3 $\beta-(-S 4)$ (cDNA from Addgene plasmid \#58269 from Peter Scheiffele's lab). All constructs were verified by DNA sequencing. The fact that AP is enzymatically active provides a simple way to test expression and measure accurate protein concentration. The 146 recombinant APor Fc-tagged constructs used for this ELISA interaction screen were secreted into the media after transient transfection, and used in the assay without further purification. Four CSPs were not included in the ELISA-based interactome assay: CNTN1AP is not expressed in the absence of CNTN1; LRP1 and VCAN are very large proteins, and costly to synthesize; and RPTPE has a very small ectodomain making potential interactions difficult to detect using this assay.

Full-length cDNAs encoding mouse IgSF8 (BC048387) and Tenascin-R (BC138043) were purchased from Origene and Source Bioscience, respectively. Both cDNAs were cloned into the pEGFP-N1 vector (Clontech) between restriction sites HindIII and SalI to place a C-terminal EGFP tag. Full-length cDNA encoding mouse Brevican (BC052032), lacking the signal peptide, was purchased from Source Bioscience and cloned into the Fc-tagged construct between restriction sites NotI and SalI.

To generate FUGW-mGFP-T2A-HA-IgSF8 two DNA fragments were PCRamplified with overlapping sequences, full-length cDNA encoding mouse IgSF8 (BC048387) with an additional HA tag, and mGFP, including T2A and HA sequences, and inserted in the FUGW-GFP vector (Addgene \#14883) between restriction sites BamHI and EcoRI. To generate FUGW-GFP-T2A-HA-IgSF8, two DNA fragments were PCR-amplified with overlapping sequences, IgSF8 (BC048387) with additional HA tag and T2A sequences, and cytosolic GFP, including a T2A sequence, and inserted in the FUGW-GFP vector (Addgene \# 14883) between restriction sites BamHI and EcoRI.

To generate pAAV-hsyn1-mGFP-T2A-Cre, EGFP-T2A-Cre was amplified by PCR from pRetroX-GFP-T2A-Cre (Addgene \#63704) and inserted between restriction sites BamHI and HindIII of pAAV-hSyn1-ChR2-EGFP (Addgene \#58881). After, mGFP was PCR-amplified from FUGW-mGFP-T2A-HA-IgSF8 and replaced by EGFP in pAAV-hsyn1-EGFP-T2A-Cre. As a control vector, pAAV-hsyn1-mGFP was generated by inserting PCR-amplified mGFP between restriction sites BamHI and HindIII of pAAV-hSyn1-ChR2-EGFP (Addgene \#58881). The plasmid pAAV-Ef1a-DIO-hChR2(E123T/T159C)-EYFP was obtained from Addgene (\#35509). A complete list of primers used to clone these constructs is available in Supplementary Table 1.

Isolation of MF synaptosomes. For initial characterization and comparison of crude MF synaptosomes and standard hippocampal synaptosomes, we followed the protocol as described in Taupin et al. ${ }^{35}$. Briefly, hippocampi were dissected quickly in ice-cold Hank's Balanced Salt Solution (HBSS) and homogenized in homogenization buffer ( $0.32 \mathrm{M}$ sucrose, $5 \mathrm{mM}$ Trizma Base, $1 \mathrm{mM} \mathrm{MgCl} 2, \mathrm{pH}$ 7.4) with protease inhibitors (pepstatin A, leupeptin, aprotinin, and PMSF) using a Dounce homogenizer. Homogenate was spun at $1000 \times g$ to pellet MF synaptosomes with nuclei and large cell debris in pellet 1 (P1), while general small synaptosomes are in the supernatant (S1). P1 and S1 fractions were loaded on Percoll gradients (Thermo-Fisher), and spun at $32,500 \times g$ at $4{ }^{\circ} \mathrm{C}$ for $7 \mathrm{~min}$ to separate MF synaptosomes and standard synaptosomes from myelin, nuclei, or mitochondria, resulting in PI (crude MF) and SI (small hippocampal) synaptosome fractions. To prepare MF synaptosomes for sorting, we adapted the protocol of Taupin et al. using 10-12 postnatal day 28 (P28) WT mice per experiment. Hippocampi were dissected quickly in ice-cold HBSS and homogenized in homogenization buffer (0.32 M sucrose, $4 \mathrm{mM}$ Hepes, $1 \mathrm{mM} \mathrm{MgCl}$, $\mathrm{pH}$ 7.4) with protease inhibitors (pepstatin A, leupeptin, aprotinin, and PMSF) using a Dounce homogenizer. After, the homogenate was filtered through a series of cell strainers $(100,70$, and $30 \mu \mathrm{m})$ and spun at $1000 \times g$ for $10 \mathrm{~min}$ at $4{ }^{\circ} \mathrm{C}$ to prepare $\mathrm{P} 1$, which includes large MF synaptosomes, and S1. P1 was washed once by resuspension in homogenization buffer and recentrifuged as above. Supernatants S1 were pooled and spun at $15,000 \times g$ for $20 \mathrm{~min}$ at $4{ }^{\circ} \mathrm{C}$ to prepare postnuclear pellet (P2) synaptosomes. P1 and P2 were resuspended in PBS and myelin was depleted from both fractions according to the instructions of a commercial myelin-removal kit (Miltenyi Biotec), before sorting MF synaptosomes from myelin-depleted P1.

Fluorescence-activated synaptosome sorting. Myelin-depleted MF synaptosomes were prepared as described above. After, MF synaptosomes were labeled in non-permeabilizing conditions in PBS with an anti-Nectin3 monoclonal antibody (1:50; Hycult Biotech) directly conjugated with CF488A fluorophore (SigmaAldrich) after depletion of bovine serum albumin (BSA) present in the antibody storage solution, using an antibody clean-up kit (Thermo-Fisher). Immediately before running the samples in the cytometer, FM4-64 (1:400; Thermo-Fisher) was added to myelin-depleted MF synaptosomes in order to label all membranecontaining material. FM4-64/Nectin3-488-double-positive MF synaptosomes were sorted on a BD FACS Aria III using an $85 \mu \mathrm{m}$ nozzle at 45 p.s.i. The pressure differential was set to two to minimize sheer stress. Nectin3-488 was excited using a $488 \mathrm{~nm} 13 \mathrm{~mW}$ laser and detected with a 530/30 band-pass filter. FM4-64 was excited using a $561 \mathrm{~nm} 30 \mathrm{~mW}$ laser and detected with a $720 / 40$ band-pass filter. Unstained and single-stained CF488A and FM4-64 synaptosomes were analyzed to calculate autofluorescence and signal above background, respectively, and Nectin3488 and FM4-64 gates were set accordingly. Synaptosomes were identified by backgating on fluorescence using FSC and SSC, with SSC set on a five decade log scale. FM4-64/Nectin3-488-double-positive MF synaptosomes were analyzed on the BD FACS Aria III post sort to quantify the purity of the sorted population.

Protein expression. For the ELISA-based assay, proteins were expressed in Expi293 suspension-adapted cells (ThermoFisher Scientific). These cells were cultured in Expi293 ${ }^{\text {tx }}$ Expression Medium and transfection was achieved by mixing $2 \mu \mathrm{g}$ of cDNA with $6 \mu \mathrm{g}$ of polyethylenimine (PEI) and adding the mixture to two 
million cells in $0.8 \mathrm{ml}$ of medium. Conditioned media was harvested 4-6 days after transfection and used within 2 days. Fc constructs were characterized by SDS-PAGE to ensure protein integrity and confirm the MW of the sample. Fcfusion proteins were quantified by WB using a known quantity of Fc-only protein. AP-fusion proteins are quantified using calf intestinal alkaline phosphatase (CIP, New England Biolabs) activity. Briefly, the activity of $20 \mu \mathrm{l}$ of conditioned medium is compared with the activity of $1 \mu \mathrm{l}$ of CIP at room temperature (RT) after 5 min in $50 \mu \mathrm{l}$ of reaction in Expi293 medium. All of the AP-fusion proteins expressed at levels $>1 \mathrm{U} / \mu \mathrm{l}$ of CIP (where $1 \mathrm{U}=10 \mathrm{pg}$ of purified CIP).

ELISA protocol. An ELISA-based assay was used to test the binding between ectodomain- $\mathrm{Fc}$ and ectodomain-AP fusions. Each data point was reproduced in three independent experiments, and each ectodomain was tested in both orientations. Fifteen $\mu \mathrm{l}$ of a solution at $3 \mu \mathrm{g} / \mathrm{ml}$ of mouse anti-AP (IgGAb-1 clone $8 \mathrm{~B} 6.18$ Thermo Fisher Scientific; Waltham, MA) in 1× PBS was added to each well of 384well plates using an automated multichannel pipette (Viaflo Assist, Integra), sealed and incubated overnight at $4{ }^{\circ} \mathrm{C}$. The following day, plates were washed, and $5 \%$ dry milk was added as a blocking agent, which was removed after $1 \mathrm{~h}$ at RT using an automated microplate washer (HydroSpeed, Tecan). Subsequently, to each well, $9 \mu \mathrm{l}$ of ecto-AP conditioned medium containing $2 \mu \mathrm{l}$ of monoclonal mouse antihuman IgG1-HRP $(2 \mu \mathrm{g} / \mathrm{ml}$; Serotec; Raleigh, NC) was added using an automated plate copier (Viaflo96, Integra) along with $7 \mu \mathrm{l}$ of ecto-Fc culture medium. Plates were sealed and incubated for $4 \mathrm{~h}$ at RT in the dark. Plates were subsequently washed, and $15 \mu \mathrm{l}$ 1-Step Ultra TMB-ELISA HRP substrate was added using an automated multichannel pipette (Viaflo Assist, Integra); after $1 \mathrm{~h}$ incubation at RT, the absorbance at $650 \mathrm{~nm}$ was recorded with a Perkin Elmer EnSpire 2300 multimode plate reader. Finally, plates were scanned to obtain matching images of the $650 \mathrm{~nm}$ reading. Known interactors such as NRXNs/NLGNs and IgLONs/IgLONs, were among the tested pairs and worked well as positive controls. Negative control bait (AP-only protein) was used to detect false-positive interactions. Background values necessary to quantify the positive reactions were obtained by averaging at least 30 contiguous "blank" wells (within a typical plate, the mean background Abs650 for 100 negative wells was $0.07 \pm 0.027$ ). Dynamic range of a typical plate was $\sim 50$ FOB.

Cell-surface binding assay. HEK293T cells were grown in DMEM (Invitrogen) supplemented with 10\% FBS (Invitrogen), penicillin/streptomycin, and transfected with EGFP, myc-LRRTM2, IgSF8-EGFP, and Tenascin-R-EGFP plasmids using Fugene6 (Promega). Twenty-four hours after transfection, the cells were incubated with Fc, NRXN1 $\beta-F c$, IgSF8-Fc, or Tenascin-R-Fc proteins $(10 \mu \mathrm{g} / \mathrm{ml})$ in DMEM supplemented with $20 \mathrm{mM}$ Hepes (pH 7.4) for $1 \mathrm{~h}$ at RT. Following two brief washes in DMEM/20 mM Hepes $\mathrm{pH}$ 7.4, cells were fixed and immunostained using mouse anti-GFP (1:500; Santa Cruz), mouse anti-c-myc (1:1000; Santa Cruz), and the Cy3-conjugated anti-Fc antibody (1:1000; Jackson ImmunoResearch). Fluorophore-conjugated secondary antibodies were from Jackson ImmunoResearch or Invitrogen (used at 1:300 or 1:1000, respectively). The cells were imaged with a Leica SP8 confocal microscope (Leica Microsystems) using a $63 \times$ objective.

Fc-protein purification. IgSF8-Fc and Tenascin-R-Fc proteins were produced by transient transfection of HEK293T cells using PEI (Polysciences). Six hours after transfection, media was changed to OptiMEM (Invitrogen) and harvested 5 days later. Conditioned media was centrifuged, sterile-filtered, and run over a fast-flow Protein-G agarose (Thermo-Fisher) column. After extensive washing with wash buffer (50 mM Hepes $\mathrm{pH} 7.4,300 \mathrm{mM} \mathrm{NaCl}$, and protease inhibitors), the column was eluted with Pierce elution buffer. Eluted fractions containing proteins were pooled and dialyzed with PBS using a Slide-A-Lyzer (Pierce) and concentrated using Amicon Ultra centrifugal units (Millipore). The integrity and purity of the purified ecto-Fc proteins was confirmed with SDS-PAGE and Coomassie staining, and concentration was determined using Bradford protein assay.

Affinity chromatography. Affinity chromatography experiments were performed as before ${ }^{60}$. P1 crude MF synaptosomes were prepared as described above from mouse brains, without the myelin-removal step. For the preparation of P2 crude synaptosome extracts, ten P21-22 rat brains were homogenized in homogenization buffer ( $4 \mathrm{mM}$ Hepes $\mathrm{pH}$ 7.4, $0.32 \mathrm{M}$ sucrose, and protease inhibitors) using a Dounce homogenizer. Homogenate was spun at $1000 \times g$ for $10 \mathrm{~min}$ at $4^{\circ} \mathrm{C}$. Supernatant was spun at $14,000 \times g$ for $20 \mathrm{~min}$ at $4{ }^{\circ} \mathrm{C}$. P1 containing crude MF synaptosomes and P2 crude synaptosomes were resuspended in extraction buffer (50 mm Hepes pH 7.4, $0.1 \mathrm{M} \mathrm{NaCl}, 2 \mathrm{mM} \mathrm{CaCl}_{2}, 2.5 \mathrm{mM} \mathrm{MgCl}_{2}$, and protease inhibitors), extracted with $1 \%$ Triton X-100 for $2 \mathrm{~h}$, and centrifuged at $100,000 \times g$ for $1 \mathrm{~h}$ at $4{ }^{\circ} \mathrm{C}$ to pellet insoluble material. Fast-flow Protein-A sepharose beads (GE Healthcare; $250 \mu \mathrm{l}$ slurry) prebound in extraction buffer to $100 \mu \mathrm{g}$ human $\mathrm{Fc}$ or IgSF8-ecto-Fc were added to the supernatant and rotated overnight at $4{ }^{\circ} \mathrm{C}$.

Beads were packed into Poly-prep chromatography columns (BioRad) and washed with $50 \mathrm{ml}$ of high-salt wash buffer (50 mM HEPES pH 7.4, $300 \mathrm{mM} \mathrm{NaCl}$, $0.1 \mathrm{mM} \mathrm{CaCl} 2,5 \%$ glycerol, and protease inhibitors), followed by a wash with $10 \mathrm{ml}$ low-salt wash buffer (50 mM HEPES pH 7.4, $150 \mathrm{mM} \mathrm{NaCl}, 0.1 \mathrm{mM} \mathrm{CaCl}, 5 \%$ glycerol, and protease inhibitors). Bound proteins were eluted from the beads by incubation with Pierce elution buffer and TCA-precipitated overnight. The precipitate was resuspended in $8 \mathrm{M}$ urea with ProteaseMax (Promega) per the manufacturer's instruction. The samples were subsequently reduced by 20 -min incubation with $5 \mathrm{mM}$ TCEP0 (tris(2carboxyethyl)phosphine) at RT and alkylated in the dark by treatment with $10 \mathrm{mM}$ Iodoacetamide for 20 additional minutes. The proteins were digested overnight at $37^{\circ} \mathrm{C}$ with Sequencing Grade Modified Trypsin (Promega) and the reaction was stopped by acidification. MS analysis was performed as before ${ }^{60}$

Mass spectrometry. Sorted MF synaptosomes and P2 synaptosomes were collected and protein extracted, precipitated and loaded in SDS-PAGE gels. Gels were further stained with InstantBlue Protein Stain (expedeon) according to instructions. Protein lanes were cut in four sections using clean material under the laminar flow. Each section was further cut in $\sim 1 \mathrm{~mm}^{3}$ cubes, transferred to individual microcentrifuge tubes, and incubated three times in $30 \%$ ethanol at $60^{\circ} \mathrm{C}$ for $20 \mathrm{~min}$ to wash excess InstantBlue Protein Stain. To prepare samples for MS, each gel section was brought to RT, and submerged in $100 \mu \mathrm{l}$ buffer containing ammonium bicarbonate at $50 \mathrm{mM}$ and TCEP at $10 \mathrm{mM}$ for cystine reduction. The gel samples were incubated in this solution at $37^{\circ} \mathrm{C}$ for $1 \mathrm{~h}$ while vortexing. The liquid was removed, and replaced with $100 \mu \mathrm{l}$ of a new solution of $50 \mathrm{mM}$ ammonium bicarbonate and $50 \mathrm{mM}$ iodoacetamide for cystine alkylation. The gel samples were then incubated in the solution at RT in the dark for $45 \mathrm{~min}$. Then, the liquid was removed and replaced with $100 \mu \mathrm{l}$ of $50 \mathrm{mM}$ ammonium bicarbonate and $50 \mathrm{mM}$ TCEP to quench alkylation reaction at RT for $30 \mathrm{~min}$. The liquid was then removed, and the gel sections were washed twice with a solution of $50 \mathrm{mM}$ ammonium bicarbonate. The gel sections were then each submerged by a $100 \mu \mathrm{l}$ solution of $50 \mathrm{mM}$ ammonium bicarbonate and $1 \mu \mathrm{g}$ of trypsin, and incubated overnight at $37^{\circ} \mathrm{C}$ with vortexing for protein digestion to peptides. The next day, the liquid from the digested gel samples was collected in new microcentrifuge tubes. Then the gel slices were treated a total of three times with a solution of $50 \%$ acetonitrile, $5 \%$ formic acid for $20 \mathrm{~min}$ followed by collection of the liquid in the new tube. The tubes now containing the extracted peptides from the gel slices were dried by vacuum centrifugation. The dried peptides were then resuspended in $200 \mu \mathrm{l}$ of a solution of $0.5 \%$ trifluoroacetic acid and desalted on C18 resin spin columns (Pierce), using 5\% acetonitrile, $0.5 \%$ trifluoroacetic acid to wash, and $80 \%$ acetonitrile, $0.5 \%$ formic acid buffer to elute. These desalted samples were then dried by vacuum centrifugation and resuspended in LC-MS/MS sample buffer of $5 \%$ acetonitrile, $1 \%$ formic acid.

For MS data acquisition from the samples, $3 \mu \mathrm{g}$ of peptides corresponding to each of the four gel sections of each of the two samples from each experiment was injected into a Thermo Orbitrap Fusion Mass Spectrometer equipped, with an Ultimate 3000 RSLCnano LC. Peptides were loaded onto a vented Acclaim Pepmap $100,75 \mu \mathrm{m} \times 2 \mathrm{~cm}$, nanoViper trap column, and subsequently separated using a nanoViper analytical column (Thermo-164570, $3 \mu \mathrm{m}, 100 \AA$, C18, $0.075 \mathrm{~mm}$, $500 \mathrm{~mm}$ ) and electrosprayed with stainless steel emitter tip assembled on the Nanospray Flex Ion Source with a spray voltage of 2000 V. Buffer A contained $\mathrm{H}_{2} \mathrm{O}$ with $5 \% \mathrm{ACN}$ and $0.125 \% \mathrm{FA}$, and buffer B contained $\mathrm{H} 2 \mathrm{O}$ with $95 \% \mathrm{ACN}$ and $0.125 \% \mathrm{FA}$. The chromatographic run was for $160 \mathrm{~min}$ in total with the following profile of percent buffer B: 0 to $8 \%$ for $6 \mathrm{~min}$, to $24 \%$ for $64 \mathrm{~min}$, to $36 \%$ for $20 \mathrm{~min}$, to $55 \%$ for $10 \mathrm{~min}$, to $95 \%$ for $10 \mathrm{~min}$, held at $95 \%$ for $20 \mathrm{~min}$, then brought down to $2 \%$ over $1 \mathrm{~min}$, and held at $2 \%$ for $29 \mathrm{~min}$. Additional MS parameters include: ion transfer tube temp at $30{ }^{\circ} \mathrm{C}$, positive spray voltage at $2500 \mathrm{~V}$, and top speed with a cycle time of $3 \mathrm{~s}$. MS1 scan in Orbitrap with $120 \mathrm{~K}$ resolution, scan range $=300-1800(\mathrm{~m} / \mathrm{z}), \max$ injection time $=50 \mathrm{~ms}$, AGC target $=100,000$, microscans $=1, \mathrm{RF}$ lens $=60 \%$, and datatype $=$ centroid. MIPS mode $=$ peptide, included charge states $=2-8$ (reject unassigned). Dynamic exclusion enabled with $n=1$ for $30 \mathrm{~s}$ exclusion duration at 5 p.p.m. for high and low. Precursor selection decision for MS2 scan in the ion trap set at most intense with a 1000 minimum intensity threshold, isolation window $=1.6$, scan range $=$ auto normal, first mass $=100$, collision energy $32 \%$ HCD, IT scan rate = rapid, max injection time at $50 \mathrm{~ms}$, AGC target $=10,000$, datatype $=$ centroid, inject ions for all available parallelizable time.

Spectrum raw files from samples were extracted into ms1 and ms2 files using in-house program RawConverter (http://fields.scripps.edu/downloads.php) ${ }^{116}$, and the tandem mass spectra were searched against UniProt mouse protein database (downloaded on 03-25-2014; The UniProt Consortium 2015) and matched to sequences using the ProLuCID/SEQUEST algorithm (ProLuCID ver. $3.1)^{117,118}$ with 50 p.p.m. peptide mass tolerance for precursor ions and 600 p.p. $\mathrm{m}$. for fragment ions. The search space included all fully and half-tryptic peptide candidates that fell within the mass tolerance window with no miscleavage constraint, assembled and filtered with DTASelect2 (ver. 2.1.3) ${ }^{119,120}$ through Integrated Proteomics Pipeline (IP2 v.3, Integrated Proteomics Applications, Inc., CA, USA http://www.integratedproteomics.com). To estimate peptide probabilities and false discovery rates (FDR) accurately, we used a target/decoy database containing the reversed sequences of all the proteins appended to the target database. Each protein identified was required to have a minimum of one peptide of minimal length of five amino acid residues and within 10 p.p.m. of the expected $\mathrm{m} / z$. However, this peptide had to be an excellent match with an FDR $<$ 0.001 and at least one excellent peptide match. After the peptide/spectrum matches were filtered, we estimated that the protein FDRs were $\leq 1 \%$ for each sample analysis. 
To identify the unique protein composition of MF synapses, first we filtered our protein list on the criteria that a candidate protein had to have at least three peptide identifications among the six samples. Then Log2 fold change of sorted MF synaptosomes vs the P2 synaptosome background was calculated on the basis of the measured NSAF. This measure normalizes spectral counts for a protein based on the total number of spectra in the run, the length of the protein, and the total number of peptides that comprise it ${ }^{121,122}$. Proteins that were not identified in a sample after filtering had their NSAF values imputed as 0 for all calculations. Proteins were determined significant if the Student's $t$ test $p$ value was $<0.05$ among the three replicates. A $q$ value was calculated based on Benjamini-Hochberg procedure and the highest ranked protein with a $q$ value $<0.05$ was used as a cutoff for determining high-confidence measurements at a $5 \%$ FDR.

Gene ontology analysis. To find cellular component terms overrepresented in sorted MF synaptosomes or P2 synaptosomes, we used the statistical overrepresentation test in the Panther Classification System using the mouse genome as reference (http://www.pantherdb.org/) ${ }^{123}$. To analyze the distribution of the MF synaptic proteome within major cellular components, we used the functional classification tool in Panther. To select CSPs among the MF synaptic proteome, we manually queried UniProt (https://www.uniprot.org/) for annotated proteins with transmembrane and extracellular domains, as well as secreted and ECM proteins. CSP functional class and protein domains were also manually examined using UniProt and known literature in PubMed (https://www.ncbi.nlm.nih.gov/pubmed/).

Fc pull-down assays. For pull-down assays on HEK293T cells, cells were grown in $10 \mathrm{~cm}$ dishes in DMEM (Invitrogen) supplemented with 10\% FBS (Invitrogen) and penicillin/streptomycin, and transfected with EGFP, IgSF8-EGFP, or Tenascin-REGFP expression constructs using Fugene6 (Promega). Twenty-four hours after transfection, the media was changed to OptiMEM (Invitrogen) for $2 \mathrm{~h}$. Cells were

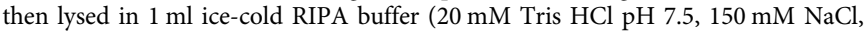
$5 \mathrm{mM}$ EDTA, $1 \%$ Triton X-100, and protease inhibitors (Roche)) for $1 \mathrm{~h}$ at $4{ }^{\circ} \mathrm{C}$ on a rocking platform. Lysates were spun at $17,000 \times g$ for $30 \mathrm{~min}$ at $4{ }^{\circ} \mathrm{C}$. Three $\mu \mathrm{g}$ of human FC, IgSF8-Fc, or Tenascin-R-Fc was added to $1 \mathrm{ml}$ of supernatant and rotated overnight at $4{ }^{\circ} \mathrm{C}$. Protein-A agarose beads $(50 \mu$ slurry) were added and rotated for $1 \mathrm{~h}$ at $4{ }^{\circ} \mathrm{C}$. Beads were washed three times in cold RIPA buffer and once in PBS, boiled in $50 \mu \mathrm{l} 2 \times$ sample buffer, and analyzed by western blotting using a mouse anti-GFP primary antibody (1:1000; Santa Cruz) and HRP-conjugated goat anti-mouse IgG secondary antibody (1:10,000; Thermo-Fisher). Uncropped and unprocessed scans are provided in Source data file.

Subcellular fractionation. Synaptic fractionation was based on a previously described method ${ }^{124}$. Ten P21 rat brains were homogenized in $12 \mathrm{ml}$ per brain with homogenization buffer $(0.32 \mathrm{M}$ sucrose, $4 \mathrm{mM}$ Hepes $\mathrm{pH} 7.4,1 \mathrm{mM} \mathrm{MgCl}$, and protease inhibitors; homogenate), centrifuged at $1500 \times g$ for $15 \mathrm{~min}$, and the supernatant was collected (postnuclear supernatant). The supernatant was then centrifuged at $18,000 \times g$ for $20 \mathrm{~min}$, and the resulting supernatant (cytosol) and pellet (crude membrane) collected. The pellet was resuspended in homogenization buffer and loaded onto $0.85 / 1.0 / 1.2 \mathrm{M}$ discontinuous sucrose gradients, and centrifuged at $78,000 \times g$ for $120 \mathrm{~min}$. The material at the $1.0 / 1.2 \mathrm{M}$ interface was collected (synaptosome). Triton X-100 was added to $0.5 \%$ and extracted at $4{ }^{\circ} \mathrm{C}$ by end-over-end agitation for $20 \mathrm{~min}$. The extract was centrifuged at $32,000 \times \mathrm{g}$ for $20 \mathrm{~min}$, the supernatant collected (soluble synaptosome/Triton-soluble fraction) and the pellet was resuspended in homogenization buffer, loaded onto a 1.0/1.5/ $2.0 \mathrm{M}$ sucrose gradient, and centrifuged at $170,000 \times g$ for $2 \mathrm{~h}$. Material was collected at the $1.5 / 2.0 \mathrm{M}$ interface (PSD). Triton X-100 (0.5\%) was added and detergent-soluble material extracted at $4{ }^{\circ} \mathrm{C}$ by end-over-end agitation for $10 \mathrm{~min}$. Lastly, the extract was centrifuged at $100,000 \times g$ for $20 \mathrm{~min}$ and the pellet resuspended in homogenization buffer (purified PSD/Triton-insoluble fraction). Primary antibodies used were the following: mouse anti-PSD95 (1:2500; ThermoFisher), rabbit ant-Synaptophysin (1:2500; Sigma-Aldrich) and goat anti-IgSF8 (1:500; R\&D Systems). HRP-conjugated secondary antibodies were from ThermoFisher (1:10000). Uncropped and unprocessed scans are provided in Source data file.

Immunocytochemistry. Crude MF (PI fraction) and small hippocampal (SI fraction) synaptosomes were spun at $15,000 \times g$ for $20 \mathrm{~min}$ at $4^{\circ} \mathrm{C}$ after being collected, resuspended in PBS, transferred to PDL-coated eight-well Nunc Lab-Tek chamberslides (Thermo-Fisher) and incubated at $4{ }^{\circ} \mathrm{C}$ on a shaker for $90 \mathrm{~min}$ to allow synaptosomes to settle. After, synaptosomes were fixed in $2 \%$ paraformaldehyde (PFA) and stained. Briefly, synaptosomes were blocked and permeabilized in 3\% BSA, $0.1 \%$ Saponin in PBS (BSA-BLOCK) at RT for 30 min and incubated with primary antibodies in BSA-BLOCK at $4{ }^{\circ} \mathrm{C}$ overnight. After, synaptosomes were washed three times with PBS, incubated with secondary antibodies at RT for $1 \mathrm{~h}$, washed three times with PBS, incubated with Hoechst dye at RT for $5 \mathrm{~min}$, washed again, and mounted with Mowiol-4-88 (MilliporeSigma Calbiochem). For live labeling, crude MF synaptosomes were incubated in nonpermeabilizing conditions with primary antibodies diluted in 3\% BSA in PBS before blocking, permeabilization, and staining of intracellular epitopes. In sorting experiments, a sample of myelin-depleted presorting P1 MF synaptosomes was collected before sorting. Remaining sample was sorted and spun at $1000 \times g$ for $10 \mathrm{~min}$ at $4{ }^{\circ} \mathrm{C}$ to pellet-sorted MF synaptosomes. Finally, sorted MF synaptosomes were resuspended in PBS and stained in permeabilizing conditions as above, in parallel to myelin-depleted presorting MF synaptosomes. Primary antibodies were the following: rat anti-Nectin3 (1:100; Hycult Biotech), mouse anti-GluK5/KA2 (1:200; NeuroMab), rabbit anti-Synpr (1:5000; Synaptic Systems), guinea pig antiVGluT1 (1:5000; Millipore), and mouse anti-PSD95 (1:250; Thermo-Fisher). Fluorophore-conjugated secondary antibodies were from Jackson ImmunoResearch or Invitrogen (used at 1:300 or 1:1000, respectively). Confocal images were acquired using a Leica SP8 confocal microscope (Leica Microsystems) and analyzed with Fiji125.

Immunohistochemistry. P28 WT mice were anesthetized by intraperitoneal injection with a lethal dose of $1 \mu \mathrm{l} / \mathrm{g}$ xylazine (VMB Xyl-M 2\%), $2 \mu \mathrm{l} / \mathrm{g}$ ketamine (Eurovet Nimatek, $100 \mathrm{mg} / \mathrm{ml}$ ), and $3 \mu \mathrm{l} / \mathrm{g} 0.9 \%$ saline. Next, mice were transcardially perfused with $4 \%$ PFA, in PBS. Brains were dissected, postfixed in $4 \%$ PFA in PBS at $4{ }^{\circ} \mathrm{C}$ for $1 \mathrm{~h}$, and cryopreserved in $30 \%$ sucrose overnight at $4{ }^{\circ} \mathrm{C}$. After, perfused brains were embedded in Tissue-Tek O.C.T. compound (Sakura) and frozen in isopentane at -55 to $-65^{\circ} \mathrm{C}$. For fresh frozen immunohistochemistry, mice were euthanized using isoflurane (Halocarbon). Next, brains were quickly dissected, embedded in Tissue-Tek O.C.T. compound (Sakura), and frozen in isopentane at -55 to $-65^{\circ} \mathrm{C}$. To prepare hippocampal sections, we used the cryostats (NX70, Thermo Fisher) and CM3050 S (Leica Biosystems). Frozen brains were cut and 16-20 $\mu \mathrm{m}$ thick coronal sections were collected on SuperFrost Ultra Plus adhesion slides (Thermo-Fisher). The fresh frozen sections were postfixed with 1:1 acetone-methanol for $5 \mathrm{~min}$ at $-20{ }^{\circ} \mathrm{C}$ and quickly washed in PBS before staining.

In some cases, heat-induced antigen retrieval ( $\mathrm{H}-\mathrm{AR})$ was done before immunostainings in sections of PFA perfused brains. For H-AR, sections were submerged in sodium citrate buffer containing $0.05 \%$ Tween 20 and $10 \mathrm{mM}$ sodium citrate $\mathrm{pH}$ of 6.0 , and heated in either a microwave up to boiling, for $30 \mathrm{~s}$, twice for $15 \mathrm{~s}$ and once for $10 \mathrm{~s}$ with a 1-2 min cooling period at RT between heating; or in the 2100 Antigen Retriever pressure cooker (Aptum Biologics Ltd) for $25 \mathrm{~min}$ up to $120^{\circ} \mathrm{C}$. After $\mathrm{H}-\mathrm{AR}$, sections were washed in PBS. Both fresh frozen and perfused sections were then permeabilized at RT for $20 \mathrm{~min}$ in PBS containing $0.05 \%$ Triton X-100 (Sigma). Sections were then blocked at RT for $2 \mathrm{~h}$ in PBS containing $10 \%$ normal horse serum (NHS), $0.5 \%$ Triton X-100, $10 \%$ glycine $2 \mathrm{M}, 0.02 \%$ gelatin, and, in the case of using mouse primary antibodies, 1:50 donkey anti-mouse IgG antigen-binding fragments (Jackson ImmunoResearch). Sections were then washed in PBS- $0.5 \%$ Triton X-100 at RT and incubated at $4{ }^{\circ} \mathrm{C}$ overnight with primary antibodies in PBS containing 5\% NHS, $0.5 \%$ Triton X-100, and $0.02 \%$ gelatin. Afterward, sections were washed in PBS- $0.5 \%$ Triton X-100 at RT before a $2 \mathrm{~h}$ incubation at RT, with secondary antibodies in PBS containing 5\% NHS, $0.5 \%$ Triton X-100, and $0.02 \%$ gelatin. Before mounting coverslips with Mowiol-4-88 (MilliporeSigma Calbiochem), sections were washed in PBS-0.5\% Triton X-100. Primary antibodies were the following: rat anti-Nectin3 (1:100; Hycult Biotech), mouse anti-GluK5/KA2 (1:500; NeuroMab), rabbit anti-Synpr (1:5000; Synaptic Systems), guinea pig anti-VGluT1 (1:5000; Millipore), rabbit anti-Synapsin3 (1:500 Synaptic Systems), rabbit anti-Afadin (1:500; Thermo-Fisher), chicken anti-ZnT3 (1:500; Synaptic Systems), rabbit anti-mGluR2/3 (1:500; Merck Millipore), rabbit anti-CRTAC1 (1:50; Merck Millipore), goat anti-IgSF8 (1:500; R\&D Systems), mouse anti-PSA-NCAM (1:200; Merck Millipore), rat anti-NCAM2 (1:200; R\&D Systems), sheep anti-ISLR2 (1:200; R\&D Systems), goat anti-NEGR1/Kilon (1:500; R\&D Systems), mouse anti-NPTX1 (1:200; BD Biosciences), goat anti-NRP1 (1:200; R\&D Systems), goat anti-NRP2 (1:200; R\&D Systems), sheep anti-Noelin (1:500; R\&D Systems), goat anti-PlexinA3 (1:50; Thermo-Fisher), rabbit antiROBO2 (1:200; Aviva Systems), goat anti-Neogenin (1:200; R\&D Systems), sheep anti-SALM2 (1:200; R\&D Systems), rabbit anti-FAM171A2 (1:50; ThermoFisher), mouse anti-Tenascin-R (1:200; R\&D Systems), sheep anti-Contactin 1 (1:200; R\&D Systems), goat anti-CD200 (1:100; R\&D Systems), goat anti-ICAM5 (1:200; Novus), goat anti-Kit/SCFR (1:100; R\&D Systems), mouse anti-LSAMP (1:1000; Developmental Studies Hybridoma Bank), mouse anti-Trk-C (1:200; R\&D Systems), goat anti-PlexinA1 (1:200; R\&D Systems), and goat anti-PTPRS (1:200; R\&D Systems). Fluorophore-conjugated secondary antibodies were from Jackson ImmunoResearch or Invitrogen (used at 1:300 or 1:1000, respectively).

For free-floating immunohistochemistry, mouse anesthesia, perfusion, and brain dissection were performed as described above. Postfixation was done overnight at $4{ }^{\circ} \mathrm{C}$ in PBS containing $4 \%$ PFA. After, brains were washed three times with PBS at RT and embedded in 3\% agarose. After, $80 \mu \mathrm{m}$ thick sections were collected in PBS containing $0.2 \% \mathrm{NaN} 3$ using the Vibrating Microtome 7000 (Campden Instruments LTD). Vibratome sections were washed at RT in PBS- $0.5 \%$ Triton X-100. Next, sections were blocked at $4{ }^{\circ} \mathrm{C}$ overnight in PBS containing $10 \%$ NHS, $0.5 \%$ Triton X-100, $0.5 \mathrm{M}$ glycine, and $0.2 \%$ gelatin. Thereafter, sections were incubated overweekend with primary antibodies in PBS containing 5\% NHS, $0.5 \%$ Triton X-100, and $0.2 \%$ gelatin. Sections were then washed with PBS- $0.5 \%$ Triton X-100 and incubated with the secondary antibodies in PBS containing 5\% NHS, $0.5 \%$ Triton X-100, and $0.2 \%$ gelatin, at $4{ }^{\circ} \mathrm{C}$ overnight. Subsequently, sections were washed in PBS- $0.5 \%$ Triton X-100. To stain nuclei, sections were incubated in PBS containing Hoechst (1:200) for 10 min at RT. Finally, sections were washed in PBS, collected on a microscope slide, and coverslips were mounted with Mowiol-4-88 
(MilliporeSigma Calbiochem). Primary antibodies were the following: chicken antiGFP (1:500; Aves Labs), rabbit anti-Synpr (1:2500; Synaptic Systems), and goat anti-IgSF8 (1:500; R\&D Systems). Fluorophore-conjugated secondary antibodies were from Jackson ImmunoResearch or Invitrogen (used at 1:300 or 1:1000, respectively). Images were acquired using a Leica SP8 confocal microscope (Leica Microsystems) or Slide Scanner Axio Scan.Z1 (Zeiss) and analyzed with Fiji ${ }^{125}$.

Western blots. Sorted MF synaptosomes were collected into sorting tubes containing $4 \times$ lysis buffer ( $40 \mathrm{mM}$ Hepes, $600 \mathrm{mM} \mathrm{NaCl}, 4 \% \mathrm{NP}-40,4 \%$ sodium deoxycholate, $0.4 \%$ SDS, and $20 \mathrm{mM}$ EDTA). P2 synaptosomes were resuspended in $2 \times$ lysis buffer, and both samples incubated on ice for $30 \mathrm{~min}$ and further rotated at $4{ }^{\circ} \mathrm{C}$ for $1 \mathrm{~h}$. To precipitate proteins, $20 \%(\mathrm{v} / \mathrm{v})$ of freshly prepared TCA was added to the lysate of both samples. After, lysates were vortexed and incubated on ice overnight in the cold room. Finally, precipitates were spun at $13,000 \times g$ at $4{ }^{\circ} \mathrm{C}$ for $30 \mathrm{~min}$, washed three times with ice-cold acetone and air-dried. Protein precipitates were then resuspended in $4 \times$ Laemmli buffer ( $8 \%$ SDS, $40 \%$ glycerol, $20 \%$ $\beta$-mercaptoethanol, $0.01 \%$ bromophenol blue, and $250 \mathrm{mM}$ Tris $\mathrm{HCl} \mathrm{pH} \mathrm{6.8),} \mathrm{pH}$ adjusted with $1.5 \mathrm{M}$ Tris $\mathrm{HCl} \mathrm{pH} \mathrm{8.8,} \mathrm{spun,} \mathrm{boiled} \mathrm{at} 95^{\circ} \mathrm{C}$ for $5 \mathrm{~min}$, and loaded in a gel for SDS-PAGE. Primary antibodies were the following: mouse anti-PSD95 (1:2500; Thermo-Fisher), rabbit ant-Synaptophysin (1:2500; Sigma-Aldrich), rabbit anti-Synpr (1:2500; Synaptic Systems), rabbit anti-Nectin3 (1:500; Abcam), goat anti-MBP (1:500; Santa Cruz), rabbit anti-Histone H3 (1:1000; Cell Signaling Technology), goat anti-IgSF8 (1:2000; R\&D Systems), mouse anti-TenR (1:1000; R\&D Systems), rabbit anti-BRINP2 (1:100; Atlas Antibodies), sheep anti-CNTN1 (1:500; R\&D Systems), goat anti-ICAM5 (1:500; R\&D Systems), sheep anti-ISLR2 (1:1000; R\&D Systems), goat anti-Kit/SCFR (1:1000; R\&D Systems), goat antiNEGR1 (1:1000; R\&D Systems), goat anti-Neogenin (1:1000; R\&D Systems), goat anti-NRP1 (1:500; R\&D Systems), goat anti-PlexinA1 (1:500; R\&D Systems), rabbit anti-PTPRD (1:500; Novus), goat anti-PTPRS (1:1000; R\&D Systems), rabbit antiROBO2 (1:1000; Abcam), and sheep anti-Teneurin-4 (1:1000; R\&D Systems). HRP-conjugated secondary antibodies were from Thermo-Fisher $(1: 10,000)$. Uncropped and unprocessed scans are provided in Source data file.

Direct binding assay. For direct binding assays of IgSF8 to Tenascin- $\mathrm{R}$ and Brevican, $1 \mu \mathrm{g}$ recombinant His-tagged mouse IgSF8 (Sino Biological) was incubated in $1 \mathrm{ml}$ binding buffer (10 mM HEPES pH 7.4, $150 \mathrm{mM} \mathrm{NaCl}, 2 \mathrm{mM} \mathrm{CaCl}_{2}$, $1 \mathrm{mM} \mathrm{MgCl}$, and $0.1 \%$ Tween-20) with equimolar amounts of control $\mathrm{Fc}$-protein (Jackson ImmunoResearch), purified Tenascin-R-Fc or Brevican-Fc, and rotated end-over-end for $1 \mathrm{~h}$ at RT. Protein-A/G agarose beads ( $100 \mu \mathrm{l}$ slurry; Santa Cruz Biotechnology) were added and rotated end-over-end for $1 \mathrm{~h}$ at $4{ }^{\circ} \mathrm{C}$. Beads were washed $4 \times$ in binding buffer and $1 \times$ in PBS, and boiled in $50 \mu \mathrm{L} 2 \times$ sample buffer. Samples were analyzed by WB. Rabbit anti-6x-His Tag primary antibody (1:250; Thermo-Fisher) and HRP-conjugated anti-rabbit secondary antibody (1:10,000; Thermo-Fisher) were used to detect IgSF8-His. Uncropped and unprocessed scans are provided in Source data file.

Biolayer Interferometry analysis. BLI binding experiments were conducted using a BLItz instrument (ForteBio, Menlo Park, CA) at RT. Anti-human Fc capture Biosensors were pre-wetted for $10 \mathrm{~min}$ in $300 \mathrm{ml}$ of $10 \mathrm{mM}$ Hepes, pH 7.4, $150 \mathrm{mM}$ $\mathrm{NaCl}, 1 \mathrm{mM} \mathrm{CaCl} 2,0.2 \%$ Tween 20 , and $1 \%(\mathrm{w} / \mathrm{v}) \mathrm{BSA}$ prior to use. Subsequently, the sensor tips were incubated for $10 \mathrm{~min}$ with conditioned medium of EXPI293 cells transfected with TenR-Fc. The binding reaction occurred under agitation in a $4 \mathrm{ml}$ drop containing various concentrations of purified proteins. Both association and dissociation were allowed to occur for $120 \mathrm{~s}$. Technical triplicates were performed for each concentration and averages were used for the final calculations. Nonspecific binding and instrument noise were subtracted by using a sensor tip saturated with $\mathrm{Fc}$ fragment alone. A slight systemic drifting is apparent in the curves, but it did not impact the quality of the experiment as judged by the statistical quality of the plot in C. The pre-wetting buffer was used for the association step, while dissociation was performed in $20 \mathrm{mM}$ HEPES $\mathrm{pH} 8,150 \mathrm{mM}$ $\mathrm{NaCl}, 3 \mathrm{mM}$ EDTA, $0.2 \%$ Tween 20 , and $0.1 \%$ BSA.

In utero electroporation. For in vivo IgSF8 localization studies, hippocampi of E15.5-day-old embryos of timed-pregnant WT C57BL/6 J mice were unilaterally electroporated with FUGW-mGFP-T2A-HA-IgSF8 plasmid. Briefly, the dam was anesthetized with isoflurane and the uterus exposed. A solution of $1 \mu \mathrm{g} / \mu \mathrm{l}$ DNA and $0.01 \%$ fast green dye was injected into the embryonic lateral ventricle with a bevelled glass capillary. The embryo's head was then placed between the paddles of pair of platinum tweezer-type electrodes (Napagene) with the cathode lateral to the filled ventricle and seven $50 \mathrm{~ms}, 40 \mathrm{~V}$ pulses were delivered at $1 \mathrm{~Hz}$ by a ECM 830 electroporator (Harvard Apparatus). After electroporation, the uterus was replaced, the incision sutured closed and the dam allowed to give birth normally.

\section{Image acquisition and analysis of in utero electroporated mice. In utero} electroporated mice were transcardially perfused with ice-cold 4\% PFA at P28. Brains were dissected and postfixed for $1 \mathrm{~h}$ at $4{ }^{\circ} \mathrm{C}$. Brains were then embedded in $3 \%$ agarose (Sigma-Aldrich) and $60 \mu \mathrm{m}$ coronal sections were cut on a vibratome (VTS1000S, Leica Biosystems). Sections in which sparsely labeled MF axons visibly expressed GFP were immunostained with chicken anti-GFP (1:500; Aves Labs) and rat anti-HA (1:250; Roche) primary antibodies. Fluorophore-conjugated secondary antibodies were from Jackson ImmunoResearch or Invitrogen (used at 1:300 or 1:1000, respectively). Coverslips were then mounted using Prolong Gold Antifade (ThermoScientific)

Sparsely labeled MF boutons were imaged on a Leica SP8 confocal microscope (Leica Microsystems) using a $63 \times$ oil immersion objective and 1.75 zoom factor. $Z$ stack containing the complete MF bouton structure, including filopodia were acquired using a $z$-step of $1 \mu \mathrm{m}$. Images were analyzed with Fiji.

Transmission electron microscopy. To analyze crude MF synaptosomes, the P1 fraction collected from ten mice was fixed with $2 \%$ glutaraldehyde, $4 \%$ PFA, $0.2 \%$

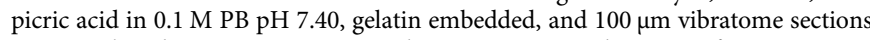
were incubated in $1 \%$ osmium tetroxide, $1.5 \%$ potassium hexacyanoferrate in $0.1 \mathrm{M}$ cacodylate buffer, and then dehydrated with ethanol. Subsequently, sections were contrasted in $2 \%$ uranyl acetate, and en block lead acetate, washed, and embedded in Epon. Samples were sectioned as $70 \mathrm{~nm}$, collected on copper grids, and examined with a Jeol Jem 1400 electron microscope.

To analyze morphological changes in Rbp4-Cre:Igsf8 cKO double transgenic mice, P30 littermates $\left(R_{b p} 4-\mathrm{Cre}^{+}: \mathrm{Igsf}^{+/+}\right.$and $\left.\mathrm{Rbp} 4-\mathrm{Cre}{ }^{+}: \mathrm{Igsf} \mathrm{g}^{\mathrm{f} / \mathrm{f}}\right)$ were transcardially perfused with $4 \%$ PFA, $2.5 \%$ glutaraldehyde, and $0.2 \%$ picric acid in $0.1 \mathrm{M}$ PB. Brains were removed, embedded in 3\% low gelling temperature agarose (Sigma-Aldrich) and $80 \mu \mathrm{m}$ sections were cut on a vibratome (VTS1000S, Leica Biosystems). Selected sections were then postfixed in a solution of $1 \% \mathrm{OsO}_{4}$ containing $1.5 \%$ potassium ferrocyanide for $60 \mathrm{~min}$ at $\mathrm{RT}$, and incubated overnight with $0.5 \%$ uranyl acetate and $25 \%$ methanol at $4{ }^{\circ} \mathrm{C}$. Then the sections were stained with Walton's lead aspartate for $30 \mathrm{~min}$ at $60^{\circ} \mathrm{C}$, dehydrated by subsequent ethanol series with increasing concentration, and finally infiltrated and embedded in resin (epon 812). Ultrathin $70 \mathrm{~nm}$ sections were cut using an ultramicrotome (Leica Biosystems EM UC7) and collected on copper grids. Sections were imaged using a JEOL JEM1400 TEM equipped with an Olympus SIS Quemesa camera operated at $80 \mathrm{kV}$. MF-CA3 synapses were identified by their morphology, presence of multiple PSDs, and high synaptic vesicle content. Overview images were taken at $2500 \times$ to analyze bouton number and area, and $5000 \times$ to analyze ultrastructure. Images were analyzed in MIB2 software (University of Helsinki) to segment the area and perimeter of individual MF-CA3 synapses, and the number and length of PSDs and AZs. Segmented images were analyzed with ImageJ software using a custom-made script. Analysis were conducted blind to genotype.

Lentivirus production. For lentivirus production, HEK293T cells were transfected with mCherry or Cre-T2A-mCherry containing FUGW vector plasmids, and helper plasmids PAX2 and VSVG using Fugene6 (Promega). Supernatant was collected $48 \mathrm{~h}$ after transfection, spun at $1000 \times g$ to remove debris and filtered through a $0.22 \mu \mathrm{m}$ filter (Millipore). A total of $200 \mu \mathrm{l}$ aliquots were stored at $-80^{\circ} \mathrm{C}$.

Adeno-associated virus production and purification. HEK293T cells were seeded in DMEM (Invitrogen) containing 10\% FBS (Invitrogen). Transfection mix, containing PEI and OptiMEM (Invitrogen), was incubated with OptiMEM containing p $\triangle \mathrm{F} 6$ helper, pAAV V2/9 Rep/Cap and pAAV-hsyn1-mGFP-T2A-Cre, pAAV-hsyn1-mGFP or pAAV-Ef1a-DIO-hChR2(E123T/T159C)-EYFP plasmids for 20 min at RT. Prior to adding DNA:PEI mix to HEK293T cells, DMEM-10\% FBS was carefully replaced with DMEM-1\% FBS. After 5 h, DMEM-10\% FBS was added to each plate. Three days after transfection, cells were harvested, pooled, centrifuged at $1000 \times g$ at $4{ }^{\circ} \mathrm{C}$ for $10 \mathrm{~min}$ and cell pellets lysed in lysis buffer $\left(150 \mathrm{mM} \mathrm{NaCl}\right.$ and $50 \mathrm{mM}$ Tris HCl-pH 8.5 in endotoxin free $\mathrm{H}_{2} \mathrm{O}$ ). Lysates were frozen in dry ice and ethanol, and thawed at $37^{\circ} \mathrm{C}$ in a water bath, for three times. Thereafter, supernatants were collected and Benzonase (Sigma) was added to a final concentration of $50 \mathrm{U} / \mathrm{ml}$. After an incubation of $30 \mathrm{~min}$ at $37^{\circ} \mathrm{C}$, lysates were centrifuged at $5000 \times g$ for $20 \mathrm{~min}$ at RT. Supernatant was then collected through a $0.45 \mu \mathrm{m}$ filter (Thermo-Fisher) and carefully layered onto iodixanol gradients in $25 \times 77 \mathrm{~mm}$ OptiSeal tubes (Beckman Coulter). Gradients were prepared using OptiPrep iodixanol (Sigma), $5 \mathrm{M} \mathrm{NaCl}, 5 \times \mathrm{PBS}$ with $1 \mathrm{mM} \mathrm{MgCl}_{2}$ and $2.5 \mathrm{mM} \mathrm{KCl}$ (5× PBS-MK), and sterile $\mathrm{H}_{2} \mathrm{O}$. Gradients were centrifuged at $300,000 \times g$ and $12{ }^{\circ} \mathrm{C}$ for $100 \mathrm{~min}$ in the Optima XE-100 Ultracentrifuge (Beckman Coulter). Next, AAVs were collected with an $18 \mathrm{G}$ needle (Beckman Coulter) from between the 40 and $60 \%$ layers, and diluted in $5 \mathrm{ml} 1 \times$ PBS-MK. The diluted AAVs were then desalted and concentrated by centrifugation at $5000 \times g$ for $30 \mathrm{~min}$ at $20^{\circ} \mathrm{C}$ in a prerinsed Amicon Ultra-15 filter (Millipore) in $1 \times$ PBS-MK. Finally, concentrated and desalted AAVs were washed by centrifugation at $5000 \times g$ and $20^{\circ} \mathrm{C}$ for $5 \mathrm{~min}$ with PBS containing $0.01 \%$ Pluronic F68 (Thermo-Fisher), aliquoted and stored at $-80^{\circ} \mathrm{C}$.

Stereotactic injections. For 3D reconstruction of MF boutons, Igsf $8 \mathrm{cKO}$ mouse littermates were injected with AAV vectors expressing mGFP or mGFP-T2A-Cre in the DG at P7. For electrophysiology experiments, Rbp4-Cre;Igsf 8 cKO mouse littermates were injected with AAV vectors expressing Cre-dependent hChR2 (E123T/T159C)-EYFP in the DG at P7. Prior to stereotactic injections, mice were intraperitoneally injected with $0.05 \mathrm{mg} / \mathrm{kg}$ buprenorphine (Vetergesic). After $1 \mathrm{~h}$, mice were anesthetized with $5 \%$ isoflurane and Duratears was applied to the eyes to 
prevent them from drying out. Mice were placed in a mouse stereotact (KOPF) equipped with a neonatal mouse adaptor (Stoelting). During the rest of the procedure $2.5 \%$ isoflurane was constantly administered. After shaving and disinfecting the mouse's head, local anesthesia was administered by a subcutaneous injection with $100 \mu \mathrm{l}$ lidocaine (xylocain 1\%). After $5 \mathrm{~min}$ an incision was made in the skin. Thereafter, the injector (Nanoject III Drummond) was placed at predetermined coordinates and beveled capillaries that penetrate skull and skin were used. One minute after lowering the capillary into the brain, viral mix was injected in the DG at $1 \mathrm{nl} / \mathrm{s}$. After a $5 \mathrm{~min}$ recovery, the capillary was pulled out at $\sim 0.1 \mathrm{~mm} / 5 \mathrm{~s}$. The incision was stitched with surgical glue (Millpledge Veterinary). After $6 \mathrm{~h}$, their health was examined and mice were injected with $0.1 \mathrm{mg} / \mathrm{kg}$ buprenorphine.

Image acquisition and analysis of injected mice. Injected mice were perfused at P28 and brains dissected, postfixed, embedded, and sectioned as before. Sections in which sparsely labeled MF axons visibly expressed GFP were immunostained with a chicken anti-GFP primary antibody (1:500; Aves Labs) and fluorophoreconjugated anti-chicken secondary antibody (1:1000; Invitrogen). Coverslips were then mounted using Mowiol-4-88 (MilliporeSigma Calbiochem). Sparsely labeled MF boutons were imaged on a Leica SP8 confocal microscope (Leica Microsystems) using a $63 \times$ oil immersion objective and 1.75 zoom factor. $Z$-stacks containing the complete MF bouton structure and filopodia were acquired using a $z$-step of $0.2 \mu \mathrm{m}$. Images were acquired from the CA3 hippocampal subregion. Acquired $Z$-stacks were then individually opened in Fiji ${ }^{125}$ and registered to correct possible drift in $z$-axis using the StackReg plugin. In order to reconstruct individual MF boutons, aligned stacks were then uploaded in Imaris (Bitplane). The number of filopodia was manually measured in 3D and only filopodia with a minimum length of $1.5 \mu \mathrm{m}$ were counted ${ }^{89}$. The 3D reconstructions of the MF bouton core were obtained by initially segmenting the volume containing the entire MF terminal. The segmented volume was subsequently masked and the MF bouton was reconstructed using the "New Surface" tool. Analysis of the volume and surface values was acquired via the "Statistics" function. Morphological analyses were conducted blind to conditions.

Acute slice electrophysiology. Sagittal slices were prepared from postnatal day P27-35 Rbp4-Cre;Igsf8 cKO littermates. Briefly, after decapitation, the brain was quickly removed and transferred into ice-cold cutting solution (in mM): $87 \mathrm{NaCl}$, $2.5 \mathrm{KCl}, 1.25 \mathrm{NaH}_{2} \mathrm{PO}_{4}, 10$ glucose, $25 \mathrm{NaHCO}_{3}, 0.5 \mathrm{CaCl}_{2}, 7 \mathrm{MgCl}_{2}, 75$ sucrose, 1 kynurenic acid, 5 ascorbic acid, 3 pyruvic acid, pH 7.4 with $5 \% \mathrm{CO}_{2} / 95 \% \mathrm{O}_{2}$ ), and whole brain sagittal slices $(300 \mu \mathrm{m})$ were cut using a vibratome (VT1200, Leica Biosystems). Afterward, slices were transferred to $34^{\circ} \mathrm{C}$ cutting solution for $45 \mathrm{~min}$ to recover and finally maintained at RT until used for recordings.

For recordings, brain slices were continuously perfused in a submerged chamber (Warner Instruments) at a rate of 3-4 ml/min with (in mM): $119 \mathrm{NaCl}, 2.5 \mathrm{KCl}, 1$ $\mathrm{NaH}_{2} \mathrm{PO}_{4}, 26 \mathrm{NaHCO}_{3}, 4 \mathrm{MgCl}_{2}, 4 \mathrm{M} \mathrm{CaCl}_{2}, 11$ glucose at $\mathrm{pH} 7.4$ with $5 \% \mathrm{CO}_{2} /$ $95 \% \mathrm{O}_{2}$. For paired-pulse and spontaneous release recordings, $20 \mu \mathrm{M}$ bicuculline, $100 \mu \mathrm{M}$ AP-5, and $150 \mathrm{nM}$ CNQX was added to the ACSF. Whole-cell patch-clamp recordings were done using borosilicate glass recording pipettes (resistance 3.5-5 M $\Omega$ ). For paired-pulse and spontaneous release experiments, we used the following internal solution (in mM): 115 CsMSF, 20 CsCl, 10 HEPES, $2.5 \mathrm{MgCl}_{2}, 4$ ATP, 0.4 GTP, 10 creatine phosphate, and 0.6 EGTA (pH 7.25). For E/I ratios, we used (in mM): $132 \mathrm{CsMSF}, 8 \mathrm{CsCl}, 10$ HEPES, $0.5 \mathrm{mM} \mathrm{CaCl}_{2}, 1$ EGTA, 10 glucose, and 5 QX-314 (pH 7.3). For experiments testing eEPSP-induced action potential generation, we used the following internal medium (in $\mathrm{mM}$ ): 135 KGluconate, 4 $\mathrm{KCl}, 2 \mathrm{NaCl}, 10$ HEPES, 4 EGTA, 4 MgATP, and 0.3 NaATP (pH 7.25).

Spontaneous and evoked input to CA3 pyramidal neurons was recorded by wholecell voltage-clamp recordings $\left(V_{\mathrm{m}}=-70 \mathrm{mV}\right)$ from visually identifiable CA3 pyramidal neurons, using a Multiclamp 700B amplifier (Axon Instruments). Spontaneous input was analysed using Mini Analysis program (Synaptosoft), evoked data were analyzed using Clampfit 10.7 (Axon Instruments). Stereotactic injections of pAVV-Ef1a-DIO-ChR2-EYFP in the DG of Rbp4-Cre;Igsf8 cKO littermates at P7 resulted in expression of ChR2 only in GCs of the DG, and therefore allowed lightinduced activation specifically of the MF pathway. We used site-directed, regioncontrolled activation (UGA-42 Geo of Rapp OptoElectronics) of MFs within SL area close to the recorded neurons. We typically needed $2-10 \%$ laser power $(0.5-2.6 \mathrm{~mW}$ with open aperture) for $1-2 \mathrm{~ms}$, using circular area selections between 40 and 120 (32-96 $\mu \mathrm{m}$ diameter) to elicit baseline eEPSCs responses (300-400 pA). Recordings requiring significantly more optical stimulation to elicit eEPSCs were discarded. For paired-pulse ratio experiments $\left(V_{\mathrm{m}}=-70 \mathrm{mV}\right)$, paired optical stimulations (interstimulus interval (ISI): 25, 50, 100, 200, 400, 1000, and $2000 \mathrm{~ms}$ ) were delivered every $20 \mathrm{~s}$ (each ISI was repeated four times) and calculated as the eEPSC2/eEPSC1 ratio. Inhibition/excitation ratios were recorded by separating eEPSCs or eIPSCs using optical stimulation when the CA3 neuron was clamped at the reversal potential of the inhibitory $(-70 \mathrm{mV})$ or excitatory $(0 \mathrm{mV})$ input, respectively. Single eEPSCs and eIPSCs were repeated eight times, every $20 \mathrm{~s}$ and averaged. For testing eEPSP-induced action potential generation, we established a basal eEPSC amplitude of $\sim 400 \mathrm{pA}$, while keeping the neuron at the chloride reversal potential $\left(V_{\mathrm{m}}=-82.6 \mathrm{mV}\right)$. Subsequently, while recorded neurons were in current clamp (Vrest), train stimulations $(10 \mathrm{~Hz}$, 20 stimulations) were used to elicit MF activation to monitor CA3 pyramidal cell excitability. For all measurements, we performed a minimum of three independent preparations.
Quantification and statistical analysis. Data analysis were performed in GraphPad Prism 8 (GraphPad software), Clampfit 10.7 (Axon Instruments), MiniAnalysis (Synaptosoft), and Fiji (NIH). For quantification, datasets were tested for normality using D'Agostino and Pearson test. If datasets passed the test, they were analyzed using Student's unpaired $t$ test. Otherwise, the datasets were analyzed using nonparametric unpaired $t$ tests (Mann-Whitney).

Resource table

\begin{tabular}{|c|c|c|}
\hline $\begin{array}{l}\text { Antibodies } \\
\text { Chicken anti-GFP }\end{array}$ & Aves Labs & $\begin{array}{l}\text { Cat\#: GFP-1010; } \\
\text { RID: AB 2307313 }\end{array}$ \\
\hline Mouse anti-GFP clone B-2 & Santa Cruz & $\begin{array}{l}\text { RID: AB_2307313 } \\
\text { Cat:: SC-9996; } \\
\text { RRID: AB 627695 }\end{array}$ \\
\hline Mouse anti-c-myc clone 9E10 & Santa Cruz & $\begin{array}{l}\text { Cat\#: SC- } 40 ; \\
\text { RRID: AB } 627268\end{array}$ \\
\hline Rabbit anti-6x-His Tag & Thermo-Fisher & $\begin{array}{l}\text { Catt: PA1-983B; } \\
\text { RRID: AB_1069891 }\end{array}$ \\
\hline Goat anti-MBP clone D-18 & Santa Cruz & $\begin{array}{l}\text { Cat\#: SC-13912; } \\
\text { RRID: AB } 648794\end{array}$ \\
\hline Mouse anti-PSD95 clone 7E3-1B8 & Thermo-Fisher & $\begin{array}{l}\text { Cat\#: MA1-046; } \\
\text { RRID: AB_2092361 }\end{array}$ \\
\hline Rabbit anti-Histone $\mathrm{H} 3$ clone $\mathrm{D} 1 \mathrm{H} 2$ & $\begin{array}{l}\text { Cell Signaling } \\
\text { Technology }\end{array}$ & $\begin{array}{l}\text { Cat\#: } 4499 ; \\
\text { RRID: AB } 10544537\end{array}$ \\
\hline Rabbit anti-Synaptophysin clone SVP-38 & Sigma-Aldrich & $\begin{array}{l}\text { Cat\#: S5768; } \\
\text { RRID: AB_477523 }\end{array}$ \\
\hline Rat anti-Nectin3 clone $103-\mathrm{A} 1$ & Hycult Biotech & $\begin{array}{l}\text { Cat\#: HM1053; } \\
\text { RRID: AB_533278 }\end{array}$ \\
\hline Rabbit anti-Nectin3 & Abcam & $\begin{array}{l}\text { Cat\#: AB63931; } \\
\text { RRID: AB_1142394 }\end{array}$ \\
\hline Mouse anti-GluK5/KA2 clone N279B/27 & NeuroMab & $\begin{array}{l}\text { Cat\#: 75-362; } \\
\text { RRID: AB_2315855 }\end{array}$ \\
\hline Rabbit anti-Synaptoporin & Synaptic Systems & $\begin{array}{l}\text { Cat\#: } 102002 ; \\
\text { RRID: AB } 887841\end{array}$ \\
\hline Guinea pig anti-VGluT1 & Merck Millipore & $\begin{array}{l}\text { Catt: AB5905; } \\
\text { RRID: AB 2301751 }\end{array}$ \\
\hline Rabbit anti-Synapsin3 & Synaptic Systems & $\begin{array}{l}\text { Cat\#: } 106 \text { 303; } \\
\text { RRID: AB_2619775 }\end{array}$ \\
\hline Rabbit anti-Afadin clone A7L9H48 & Thermo-Fisher & $\begin{array}{l}\text { Catt: } 700193 ; \\
\text { RRID: AB_2532299 }\end{array}$ \\
\hline Chicken anti-ZnT3 & Synaptic Systems & $\begin{array}{l}\text { Catt: } 197 \text { 006; } \\
\text { RRID: AB_2725754 }\end{array}$ \\
\hline Rabbit anti-mGluR2/3 & Merck Millipore & $\begin{array}{l}\text { Cat\#: AB1553; } \\
\text { RRID: AB_11212089 }\end{array}$ \\
\hline Rabbit anti-CRTAC1 & Merck Millipore & Cat\#: ABD80 \\
\hline Rabbit anti-BRINP2 & Atlas Antibodies & $\begin{array}{l}\text { Cat\#: HPA061920; } \\
\text { RRID: AB_2684639 }\end{array}$ \\
\hline Goat anti-IgSF8 & R\&D Systems & $\begin{array}{l}\text { Cat\#: AF3117; } \\
\text { RRID: AB_2233385 }\end{array}$ \\
\hline Rabbit anti-FAM171A2 & ThermoFisher & $\begin{array}{l}\text { Catt: PA5-71105; } \\
\text { RRID: AB_2689922 }\end{array}$ \\
\hline Rabbit anti-RPTPd & Novus & Cat\#: NBP2-49153 \\
\hline Goat anti-RPTPs & R\&D Systems & $\begin{array}{l}\text { Cat\#: AF3430; } \\
\text { RRID: AB_2175157 }\end{array}$ \\
\hline Sheep anti-Teneurin-4 & R\&D Systems & $\begin{array}{l}\text { Cat\#: AF6320; } \\
\text { RRID: AB_10920937 }\end{array}$ \\
\hline Mouse anti-PSA-NCAMI & Merck Millipore & $\begin{array}{l}\text { Catt: MAB5324; } \\
\text { RRID: AB_95211 }\end{array}$ \\
\hline Rat anti-NCAM2 & R\&D Systems & $\begin{array}{l}\text { Cat\#: MAB778; } \\
\text { RRID: AB_2149709 }\end{array}$ \\
\hline Sheep anti-ISLR2 & R\&D Systems & $\begin{array}{l}\text { Catt: AF4650; } \\
\text { RRID: AB_2126610 }\end{array}$ \\
\hline Goat anti-NEGR1/Kilon & R\&D Systems & $\begin{array}{l}\text { Catt: AF5394; } \\
\text { RRID: AB_2150086 }\end{array}$ \\
\hline Mouse anti-Neuronal pentraxin 1 & BD Biosciences & $\begin{array}{l}\text { Cat\#: 610369; } \\
\text { RRID: AB_397754 }\end{array}$ \\
\hline Goat anti-NRP1 & R\&D Systems & $\begin{array}{l}\text { Cat\#: AF566; } \\
\text { RRID: AB_355445 }\end{array}$ \\
\hline Goat anti-NRP2 & R\&D Systems & $\begin{array}{l}\text { Cat\#: AF2215; } \\
\text { RRID: AB_2155371 }\end{array}$ \\
\hline Sheep anti-Noelin & R\&D Systems & $\begin{array}{l}\text { Cat\#: AF4636; } \\
\text { RRID: AB_2157225 }\end{array}$ \\
\hline Goat anti-Plexin-A3 & Thermo-Fisher & $\begin{array}{l}\text { Cat\#: PA5-47571; } \\
\text { RRID: AB_2608296 }\end{array}$ \\
\hline Rabbit anti-ROBO2 & Aviva Systems & $\begin{array}{l}\text { Cat\#: ARP45396_P050; } \\
\text { RRID: AB_2047840 }\end{array}$ \\
\hline Goat anti-Neogenin & R\&D Systems & $\begin{array}{l}\text { Catt: AF1079; } \\
\text { RRID: AB_2151002 }\end{array}$ \\
\hline Sheep anti-SALM2 & R\&D Systems & $\begin{array}{l}\text { Catt: AF5669; } \\
\text { RRID: AB_1964706 }\end{array}$ \\
\hline Mouse anti-Tenascin- $R$ & R\&D Systems & $\begin{array}{l}\text { Cat\#: MĀB1624; } \\
\text { RRID: AB_2207001 }\end{array}$ \\
\hline Sheep anti-Contactin 1 & R\&D Systems & Catt: AF7549 \\
\hline Goat anti-CD200 & R\&D Systems & Cat\#: AF2724 \\
\hline Goat anti-ICAM5 & Novus & $\begin{array}{l}\text { Catt: NB100-53815; } \\
\text { RRID: AB_829163 }\end{array}$ \\
\hline Goat anti-SCFR/Kit & R\&D Systems & $\begin{array}{l}\text { Cat\#: AF1356; } \\
\text { RRID: AB_354750 }\end{array}$ \\
\hline Mouse anti-LSAMP & $\begin{array}{l}\text { Developmental } \\
\text { Studies } \\
\text { Hybridoma Bank }\end{array}$ & $\begin{array}{l}\text { Cat\#: 2G9; } \\
\text { RRID: AB_2138210 }\end{array}$ \\
\hline Mouse anti-Trk-C & R\&D Systems & $\begin{array}{l}\text { Cat\#: MAB373; } \\
\text { RRID: AB_2155422 }\end{array}$ \\
\hline Goat anti-Plexin-A1 & R\&D systems & $\begin{array}{l}\text { Cat\#: AF4309; } \\
\text { RRID: AB_10645644 }\end{array}$ \\
\hline Rat anti-HA & Roche & $\begin{array}{l}\text { Cat\#: 118674223001; } \\
\text { RRID: AB } 390918\end{array}$ \\
\hline Rabbit anti-pERM & Cell Signaling & $\begin{array}{l}\text { Cat\#: 3726; } \\
\text { RRID:AB } 10560513\end{array}$ \\
\hline Goat anti-Chicken Alexa 488 & Thermo-Fisher & $\begin{array}{l}\text { Cat\# A-11039; } \\
\text { RRID: AB_2534096 }\end{array}$ \\
\hline
\end{tabular}




\begin{tabular}{|c|c|c|}
\hline Donkey anti-Goat Alexa 488 & Thermo-Fisher & $\begin{array}{l}\text { Cat\#: A-11055; } \\
\text { RRID: AB_2534102 }\end{array}$ \\
\hline Donkey anti-Goat Alexa 647 & Thermo-Fisher & $\begin{array}{l}\text { Cat\#: A-21447; } \\
\text { RRID: AB_2535864 }\end{array}$ \\
\hline Donkey anti-Rabbit Alexa 488 & Thermo-Fisher & $\begin{array}{l}\text { Cat\#: A-21206; } \\
\text { RRID: AB_2535792 }\end{array}$ \\
\hline Donkey anti-Rabbit Alexa 555 & Thermo-Fisher & $\begin{array}{l}\text { Cat\#: A-31572; } \\
\text { RRID: AB_162543 }\end{array}$ \\
\hline Donkey anti-Rabbit Alexa 647 & Thermo-Fisher & $\begin{array}{l}\text { Cat\#: A-31573; } \\
\text { RRID: AB_2536183 }\end{array}$ \\
\hline Donkey anti-Mouse Alexa 488 & Thermo-Fisher & $\begin{array}{l}\text { Cat\#: A21202; } \\
\text { RRID: AB_141607 }\end{array}$ \\
\hline Donkey anti-Mouse Alexa 555 & Thermo-Fisher & $\begin{array}{l}\text { Cat\#: A31570; } \\
\text { RRID: AB_2536180 }\end{array}$ \\
\hline Donkey anti-Mouse Alexa 647 & Thermo-Fisher & $\begin{array}{l}\text { Cat\#: A-31571; } \\
\text { RRID: AB_162542 }\end{array}$ \\
\hline Donkey anti-Sheep Alexa 488 & Thermo-Fisher & $\begin{array}{l}\text { Cat\# A-11015; } \\
\text { RRID: AB_2534082 }\end{array}$ \\
\hline Donkey anti-Sheep Alexa 647 & Thermo-Fisher & $\begin{array}{l}\text { Cat\#: A-21448; } \\
\text { RRID: AB_2535865 }\end{array}$ \\
\hline Donkey anti-Chicken Alexa 488 & $\begin{array}{l}\text { Jackson } \\
\text { ImmunoResearch }\end{array}$ & $\begin{array}{l}\text { Cat\# 703-545-155; } \\
\text { RRID: AB_2340375 }\end{array}$ \\
\hline Donkey anti-Rat Alexa 647 & $\begin{array}{l}\text { Jackson } \\
\text { ImmunoResearch }\end{array}$ & $\begin{array}{l}\text { Cat\#: 712-605-153; } \\
\text { RRID: AB_2340694 }\end{array}$ \\
\hline Donkey anti-Guinea Pig Cy3 & $\begin{array}{l}\text { Jackson } \\
\text { ImmunoResearch }\end{array}$ & $\begin{array}{l}\text { Cat\# 706-165-148; } \\
\text { RRID: AB_2340460 }\end{array}$ \\
\hline Donkey anti-Guinea Pig Alexa 647 & $\begin{array}{l}\text { Jackson } \\
\text { ImmunoResearch }\end{array}$ & $\begin{array}{l}\text { Cat\#: 706-605-148; } \\
\text { RRID: AB_2340476 }\end{array}$ \\
\hline Donkey anti-Human IgG, Fc Fragment Cy3 & $\begin{array}{l}\text { Jackson } \\
\text { ImmunoResearch }\end{array}$ & $\begin{array}{l}\text { Cat\#: 709-165-098; } \\
\text { RRID: AB_2340534 }\end{array}$ \\
\hline HRP Rabbit anti-Goat IgG & Thermo-Fisher & $\begin{array}{l}\text { Cat\#: 81-1620; } \\
\text { RRID: AB_2534006 }\end{array}$ \\
\hline HRP Rabbit anti-Sheep IgG & Thermo-Fisher & $\begin{array}{l}\text { Cat\#: } 61-8620 ; \\
\text { RRID: AB_2533942 }\end{array}$ \\
\hline HRP Goat anti-Mouse IgG & Thermo-Fisher & $\begin{array}{l}\text { Cat\#: 62-6520; } \\
\text { RRID: AB_2533947 }\end{array}$ \\
\hline HRP Goat anti-Rabbit IgG & Thermo-Fisher & $\begin{array}{l}\text { Cat\#: 65-6120; } \\
\text { RRID: AB_2533967 }\end{array}$ \\
\hline $\begin{array}{l}\text { Placental alkaline phosphatase monoclonal } \\
\text { antibody (8B6.18) }\end{array}$ & Thermo-Fisher & $\begin{array}{l}\text { Cat\#: MA5-12694; } \\
\text { RRID: AB } 10978663\end{array}$ \\
\hline $\begin{array}{l}\text { HRP mouse anti-Human IgG1 } \\
\text { Bacterial and virus strains }\end{array}$ & Serotec & Cat\#: MCA514P \\
\hline AAV2/9-hsyn1-mGFP & This paper & N/A \\
\hline AAV2/9-hsyn1-mGFP-T2A-Cre & This paper & N/A \\
\hline AAV2/9-Ef1a-DIO-hChR2(E123T/T159C)-EYFP & & $\begin{array}{l}\text { Cat\#: Addgene plasmid } \\
\text { \#35509; } \\
\text { RRID: Addgene_35509 }\end{array}$ \\
\hline $\begin{array}{l}\text { Chemicals, peptides, and recombinant proteins } \\
\text { IgSF8-Fc }\end{array}$ & This paper & 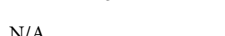 \\
\hline IgSF8-His & Sino Biological & $\begin{array}{l}\text { N/A } \\
\text { Cat\#: } 51042-\mathrm{M} 08 \mathrm{H}\end{array}$ \\
\hline Tenascin- $\mathrm{R}-\mathrm{Fc}$ & This paper & N/A \\
\hline Brevican-Fc & This paper & N/A \\
\hline Neurexin $1 \beta$-Fc & $127 \mathrm{~F}-\mathrm{P}$ & N/A \\
\hline Hoechst 33342 & Thermo-Fisher & Cat\#: H3570 \\
\hline $\mathrm{FM}^{\mathrm{st}} 4-64 \mathrm{FX}$, fixable analog & Thermo-Fisher & Cat\#: F34653 \\
\hline ChromPure Human IgG, Fc fragment & $\begin{array}{l}\text { Jackson } \\
\text { Immunoresearch }\end{array}$ & $\begin{array}{l}\text { Cat\#: 009-000-008; } \\
\text { RRID: AB_2337046 }\end{array}$ \\
\hline AffiniPure Fab Fragment Donkey anti-Mouse IgG & Jackson & Cat\#: $715-007-003$ \\
\hline$(\mathrm{H}+\mathrm{L})$ & Immunoresearch & RRID: AB_2307338 \\
\hline FuGENE 6 Transfection Reagent & Promega & Cat\#: E2691 \\
\hline AMAXA Nucleofector kit & Lonza & Cat\#:VPG-1001 \\
\hline 1-Step Ultra TMB-ELISA HRP & Thermo-Fisher & Cat\#: 34028 \\
\hline $\begin{array}{l}\text { Critical commercial assays } \\
\text { Myelin-Removal Beads II, human, mouse, rat }\end{array}$ & Miltenyi Biotec & Cat\#: 130-096-733 \\
\hline Mix-n-Stain ${ }^{\text {min }}$ CF $^{\text {mo }} 488$ A Antibody Labeling Kit & Sigma-Aldrich & $\begin{array}{l}\text { Cat\#: MX488AS100; } \\
\text { RRID: AB_10961132 }\end{array}$ \\
\hline Pierce $^{m}$ Antibody Clean-up Kit & Thermo-Fisher & Cat\#: 44600 \\
\hline Gibson Assembly Cloning Kit & $\begin{array}{l}\text { New England } \\
\text { Biolabs }\end{array}$ & Cat\#: E5510S \\
\hline Deposited data & & \\
\hline $\begin{array}{l}\text { Proteomic analysis of sorted MF synaptosomes and } \\
\text { P2 synaptosomes } \\
\text { Experimental models: cell lines }\end{array}$ & Proteome Exchange & PXD013492 \\
\hline Human: HEK293T cells & ATCC & $\begin{array}{l}\text { Cat\#: CRL-11268 } \\
\text { RRID: CVCL_1926 }\end{array}$ \\
\hline Human: HEK293F suspension adapted & Thermo-Fisher & Cat \# R790-07 \\
\hline $\begin{array}{l}\text { Mouse: hippocampal primary neurons cultured } \\
\text { from embryonic day } 18 \text { Igsf8 conditional knock } \\
\text { out mice }\end{array}$ & This paper & N/A \\
\hline $\begin{array}{l}\text { Experimental models: organisms/strains } \\
\text { Mouse: Igsf8 cKO (B6D2;129S2-Igsfgtt1.1Osb) }\end{array}$ & Riken Institute ${ }^{64}$ & $\begin{array}{l}\text { Cat\#: RBRC05637; } \\
\text { RRID: IMSR_RBRC05637 }\end{array}$ \\
\hline Mouse: $\operatorname{Tg}(\mathrm{Rbp} 4-\mathrm{cre}) \mathrm{KL} 100 \mathrm{Gsat}$ & GENSAT & $\begin{array}{l}\text { Cat\#: 031125-UCD; } \\
\text { RRID: MMRRC_031125- } \\
\text { UCD }\end{array}$ \\
\hline Mouse: $\mathrm{C} 57 \mathrm{BL} / 6 \mathrm{~J}$ & JAX & $\begin{array}{l}\text { Cat\#: 000664; } \\
\text { RRID: IMSR_JAX:000664 }\end{array}$ \\
\hline Recombinant DNA & & \\
\hline Plasmid: FUGW-mCherry & 115 & $\mathrm{~N} / \mathrm{A}$ \\
\hline Plasmid: FUGW-CRE-T2A-mCherry & 115 & N/A \\
\hline Plasmid: FUGW-mGFP-T2A-HA-IgSF8 & This paper & N/A \\
\hline Plasmid: FUGW-GFP-T2A-HA-IgSF8 & This paper & $\mathrm{N} / \mathrm{A}$ \\
\hline Plasmid: pAAV-hsynl-mGFP & This paper & N/A \\
\hline Plasmid: pAAV-hsyn1-mGFP-T2A-Cre & This paper & N/A \\
\hline $\begin{array}{l}\text { Plasmid: pAAV-Ef1a-DIO-hChR2(E123T/T159C)- } \\
\text { EYFP }\end{array}$ & & $\begin{array}{l}\text { Cat\#: Addgene plasmid } \\
\text { \#35509; RRID: } \\
\text { Addgene } 35509\end{array}$ \\
\hline Plasmid: pBOS-EGFP & 128 & N/A \\
\hline Plasmid: pBOS-myc-LRRTM2 & 127 & N/A \\
\hline Plasmid: pEGFP-N1 & Clontech (TaKaRa) & N/A \\
\hline Plasmid: pEGFP-N1-IgSF8 & This paper & N/A \\
\hline Plasmid: pEGFP-N1-Tenascin- $R$ & This paper & N/A \\
\hline Plasmid: $4 \mathrm{~F} 2 \mathrm{hc}-\mathrm{Fc} / \mathrm{AP}$ & This paper & \\
\hline Plasmid: ADAM11-FC/AP & This paper & \\
\hline Plasmid: ADAM22-Fc/AP & This paper & \\
\hline
\end{tabular}

\begin{tabular}{|c|c|c|}
\hline Plasmid: ADAM23-Fc/AP & This paper & \\
\hline Plasmid: ALCAM-Fc/AP & This paper & \\
\hline Plasmid: APMAP-Fc/AP & This paper & \\
\hline Plasmid: BRINP2-Fc/AP & This paper & \\
\hline Plasmid: CADM4-Fc/AP & This paper & \\
\hline Plasmid: CD200-Fc/AP & This paper & \\
\hline Plasmid: CNTN1-Fc/AP & This paper & \\
\hline Plasmid: CNTNAP2-Fc/AP & This paper & \\
\hline Plasmid: CRTAC1-Fc/AP & This paper & \\
\hline Plasmid: ELFN1-Fc/AP & This paper & \\
\hline Plasmid: ELFN2-Fc/AP & This paper & \\
\hline Plasmid: EPHA4-Fc/AP & This paper & \\
\hline Plasmid: EPHA6-Fc/AP & This paper & \\
\hline Plasmid: EPHA7-Fc/AP & This paper & \\
\hline Plasmid: EPHB2-Fc/AP & This paper & \\
\hline Plasmid: EPHB4-Fc/AP & This paper & \\
\hline Plasmid: FAM171A2-Fc/AP & This paper & \\
\hline Plasmid: GPR37L1-Fc/AP & This paper & \\
\hline Plasmid: HAPLN1-Fc/AP & This paper & \\
\hline Plasmid: HEPACAM-Fc/AP & This paper & \\
\hline Plasmid: ICAM5-Fc/AP & This paper & \\
\hline Plasmid: IgSF8-Fc/AP & This paper & \\
\hline Plasmid: ISLR2-Fc/AP & This paper & \\
\hline Plasmid: KIT/SCFR-Fc/AP & This paper & \\
\hline Plasmid: L1CAM-Fc/AP & This paper & \\
\hline Plasmid: LGI1-Fc/AP & This paper & \\
\hline Plasmid: LINGO1-Fc/AP & This paper & \\
\hline Plasmid: Lphn1-Fc/AP & This paper & \\
\hline Plasmid: Lphn3-Fc/AP & This paper & \\
\hline Plasmid: LRFN1/SALM2-Fc/AP & This paper & \\
\hline Plasmid: LRRC4B/NGL3-Fc/AP & This paper & \\
\hline Plasmid: LSAMP/IgLON3-Fc/AP & This paper & \\
\hline Plasmid: NCAM1-Fc/AP & This paper & \\
\hline Plasmid: NCAM2-Fc/AP & This paper & \\
\hline Plasmid: Nectin3-Fc/AP & This paper & \\
\hline Plasmid: NEGR1/IgLON4-Fc/AP & This paper & \\
\hline Plasmid: Neogenin-Fc/AP & This paper & \\
\hline Plasmid: Neurofascin-Fc/AP & This paper & \\
\hline Plasmid: NLGN1-Fc/AP & This paper & \\
\hline Plasmid: NLGN2-Fc/AP & This paper & \\
\hline Plasmid: NLGN3-Fc/AP & This paper & \\
\hline Plasmid: NPTN-Fc/AP & This paper & \\
\hline Plasmid: NPTX1-Fc/AP & This paper & \\
\hline Plasmid: Noelin-Fc/AP & This paper & \\
\hline Plasmid: NrCAM-Fc/AP & This paper & \\
\hline Plasmid: NRP1-Fc/AP & This paper & \\
\hline Plasmid: NRP2-Fc/AP & This paper & \\
\hline Plasmid: NRXN1 $\alpha-F c / A P$ & This paper & \\
\hline Plasmid: NRXN3a-Fc/AP & This paper & \\
\hline Plasmid: NRXN1 $\beta$-Fc/AP & This paper & \\
\hline Plasmid: NRXN3 3 -Fc/AP & This paper & \\
\hline Plasmid: Neurotrimin/IgLON2-Fc/AP & This paper & \\
\hline Plasmid: PlexinA1-Fc/AP & This paper & \\
\hline Plasmid: PlexinA2-Fc/AP & This paper & \\
\hline Plasmid: PlexinA3-Fc/AP & This paper & \\
\hline Plasmid: PlexinA4-Fc/AP & This paper & \\
\hline Plasmid: PlexinB1-Fc/AP & This paper & \\
\hline Plasmid: PlexinC1-Fc/AP & This paper & \\
\hline Plasmid: RPTPa-Fc/AP & This paper & \\
\hline Plasmid: RPTP $\delta$-Fc/AP & This paper & \\
\hline Plasmid: RPTPf/LAR-Fc/AP & This paper & \\
\hline Plasmid: RPTP $\sigma-F c / A P$ & This paper & \\
\hline Plasmid: ROBO1-Fc/AP & This paper & \\
\hline Plasmid: $\mathrm{ROBO} 2-\mathrm{Fc} / \mathrm{AP}$ & This paper & \\
\hline Plasmid: Shisa6-Fc/AP & This paper & \\
\hline Plasmid: SIRPa-Fc/AP & This paper & \\
\hline Plasmid: Teneurin4-Fc/AP & This paper & \\
\hline Plasmid: TenR-Fc/AP & This paper & \\
\hline Plasmid: TrkC-Fc/AP & This paper & \\
\hline Plasmid: VCAM1-Fc/AP & This paper & \\
\hline Mouse IgSF8 cDNA clone & Origene & MC206708 \\
\hline Mouse Tenascin-R cDNA clone & Source Bioscience & IRCKp5014L0710Q \\
\hline Mouse Brevican cDNA clone & Source Bioscience & IRAVp968B01111D \\
\hline Software and algorithms & & \\
\hline Fiji & $\mathrm{NIH}$ & $\begin{array}{l}\text { https://imagej.net/ } \\
\text { Welcome; }\end{array}$ \\
\hline & & RRID: SCR_002285 \\
\hline Imaris & BITPLANE & $\begin{array}{l}\text { http://www.bitplane.com/ } \\
\text { imaris; }\end{array}$ \\
\hline & & \\
\hline Microscope Image Browser (MIB) & $\begin{array}{l}\text { University of } \\
\text { Helsinki }\end{array}$ & $\begin{array}{l}\text { http://mib.helsinki.fi/index. } \\
\text { htm }\end{array}$ \\
\hline GraphPad Prism8 & GraphPad & https://www.graphpad. \\
\hline & Software, Inc & com/scientific-software/ \\
\hline & & $\begin{array}{l}\text { prism/; } \\
\text { RRID: SCR_002798 }\end{array}$ \\
\hline
\end{tabular}

Reporting summary. Further information on research design is available in the Nature Research Reporting Summary linked to this article.

\section{Data availability}

Proteomic analysis of sorted MF synaptosomes and P2 synaptosomes generated during the study is available in Proteome Exchange: PXD013492: UniProt mouse protein database (downloaded on 03-25-2014; The UniProt Consortium 2015; https://www. uniprot.org/); Panther Classification System using the mouse genome as reference (http://www.pantherdb.org/); PubMed (https://www.ncbi.nlm.nih.gov/pubmed/). Source data are provided with this paper. 
Received: 10 April 2020; Accepted: 23 September 2020;

Published online: 14 October 2020

\section{References}

1. Rao-Ruiz, P., Yu, J., Kushner, S. A. \& Josselyn, S. A. Neuronal competition: microcircuit mechanisms define the sparsity of the engram. Curr. Opin. Neurobiol. 54, 163-170 (2019).

2. Silberberg, G., Grillner, S., LeBeau, F. E. N., Maex, R. \& Markram, H. Synaptic pathways in neural microcircuits. Trends Neurosci. 28, 541-551 (2005).

3. Grillner, S., Markram, H., De Schutter, E., Silberberg, G. \& LeBeau, F. E. N. Microcircuits in action-from CPGs to neocortex. Trends Neurosci. 28, 525-533 (2005).

4. Rebola, N., Carta, M. \& Mulle, C. Operation and plasticity of hippocampal CA3 circuits: implications for memory encoding. Nat. Rev. Neurosci. 18, 208-220 (2017).

5. Chicurel, M. E. \& Harris, K. M. Three-dimensional analysis of the structure and composition of CA3 branched dendritic spines and their synaptic relationships with mossy fiber boutons in the rat hippocampus. J. Comp. Neurol. 325, 169-182 (1992).

6. Wilke, S. A. et al. Deconstructing complexity: serial block-face electron microscopic analysis of the hippocampal mossy fiber synapse. J. Neurosci. 33, 507-522 (2013).

7. Rollenhagen, A. et al. Structural determinants of transmission at large hippocampal mossy fiber synapses. J. Neurosci. 27, 10434-10444 (2007).

8. Lawrence, J. J. \& McBain, C. J. Interneuron diversity series: containing the detonation-feedforward inhibition in the CA3 hippocampus. Trends Neurosci. 26, 631-640 (2003)

9. Acsády, L., Kamondi, A., Sík, A., Freund, T. \& Buzsáki, G. GABAergic cells are the major postsynaptic targets of mossy fibers in the rat hippocampus. $J$. Neurosci. 18, 3386-3403 (1998).

10. Henze, D. A., Wittner, L. \& Buzsáki, G. Single granule cells reliably discharge targets in the hippocampal CA3 network in vivo. Nat. Neurosci. 5, 790-795 (2002).

11. Mori, M., Abegg, M. H., Gähwiler, B. H. \& Gerber, U. A frequency-dependent switch from inhibition to excitation in a hippocampal unitary circuit. Nature 431, 453-456 (2004).

12. Martin, E. A. et al. The intellectual disability gene Kirrel3 regulates targetspecific mossy fiber synapse development in the hippocampus. eLife 4, e09395 (2015).

13. Torborg, C. L., Nakashiba, T., Tonegawa, S. \& McBain, C. J. Control of CA3 output by feedforward inhibition despite developmental changes in the excitation-inhibition balance. J. Neurosci. 30, 15628-15637 (2010).

14. Guo, N. et al. Dentate granule cell recruitment of feedforward inhibition governs engram maintenance and remote memory generalization. Nat. Med. 24, 438-449 (2018).

15. Ruediger, S. et al. Learning-related feedforward inhibitory connectivity growth required for memory precision. Nature 473, 514-518 (2011).

16. Villanueva-Castillo, C., Tecuatl, C., Herrera-López, G. \& Galván, E. J. Agingrelated impairments of hippocampal mossy fibers synapses on CA3 pyramidal cells. Neurobiol. Aging 49, 119-137 (2017).

17. de Wit, J. \& Ghosh, A. Specification of synaptic connectivity by cell surface interactions. Nat. Rev. Neurosci. 17, 22-35 (2016).

18. Sanes, J. R. \& Yamagata, M. Many paths to synaptic specificity. Annu. Rev. Cell Dev. Biol. 25, 161-195 (2009).

19. Shen, K. \& Scheiffele, P. Genetics and cell biology of building specific synaptic connectivity. Annu. Rev. Neurosci. 33, 473-507 (2010).

20. Földy, C. et al. Single-cell RNAseq reveals cell adhesion molecule profiles in electrophysiologically defined neurons. Proc. Natl Acad. Sci. USA 113, E5222-E5231 (2016)

21. Li, H. et al. Classifying Drosophila olfactory projection neuron subtypes by single-cell RNA sequencing. Cell 171, 1206-1220.e22 (2017).

22. Paul, A. et al. Transcriptional architecture of synaptic communication delineates GABAergic neuron identity. Cell 171, 522-539.e20 (2017).

23. Shekhar, K. et al. Comprehensive classification of retinal bipolar neurons by single-transcriptomics. Cell Cell 166, 1308-1323.e30 (2016).

24. Südhof, T. C. Towards an understanding of synapse formation. Neuron 100, 276-293 (2018).

25. Özkan, E. et al. An extracellular interactome of immunoglobulin and LRR proteins reveals receptor-ligand networks. Cell 154, 228-239 (2013).

26. Visser, J. J. et al. An extracellular biochemical screen reveals that FLRTs and Unc5s mediate neuronal subtype recognition in the retina. Elife 4, e08149 (2015).

27. Condomitti, G. et al. An input-specific orphan receptor GPR158-HSPG interaction organizes hippocampal mossy fiber-CA3 synapses. Neuron 100 , 201-215.e9 (2018)
28. DeNardo, L. A., de Wit, J., Otto-Hitt, S. \& Ghosh, A. NGL-2 regulates inputspecific synapse development in CA1 pyramidal neurons. Neuron 76, 762-775 (2012).

29. Sando, R., Jiang, X. \& Südhof, T. C. Latrophilin GPCRs direct synapse specificity by coincident binding of FLRTs and teneurins. Science 363, eaav7969 (2019).

30. Schroeder, A. et al. A modular organization of LRR protein-mediated synaptic adhesion defines synapse identity. Neuron 99, 329-344.e7 (2018).

31. Favuzzi, E. et al. Distinct molecular programs regulate synapse specificity in cortical inhibitory circuits. Science 363, 413-417 (2019).

32. Apóstolo, N. \& de Wit, J. Compartmentalized distributions of neuronal and glial cell-surface proteins pattern the synaptic network. Curr. Opin. Neurobiol. 57, 126-133 (2019).

33. Rollenhagen, A. \& Lübke, J. H. R. The morphology of excitatory central synapses: from structure to function. Cell Tissue Res. 326, 221-237 (2006).

34. Amaral, D. G. \& Dent, J. A. Development of the mossy fibers of the dentate gyrus: I. A light and electron microscopic study of the mossy fibers and their expansions. J. Comp. Neurol. 195, 51-86 (1981).

35. Taupin, P., Zini, S., Cesselin, F., Ben-Ari, Y. \& Roisin, M. P. Subcellular fractionation on Percoll gradient of mossy fiber synaptosomes: morphological and biochemical characterization in control and degranulated rat hippocampus. J. Neurochem 62, 1586-1595 (1994).

36. Williams, M. E. et al. Cadherin-9 regulates synapse-specific differentiation in the developing hippocampus. Neuron 71, 640-655 (2011).

37. Cembrowski, M. S., Wang, L., Sugino, K., Shields, B. C. \& Spruston, N. Hipposeq: a comprehensive RNA-seq database of gene expression in hippocampal principal neurons. Elife 5, e14997 (2016)

38. Mizoguchi, A. et al. Nectin: an adhesion molecule involved in formation of synapses. J. Cell Biol. 156, 555-565 (2002)

39. Darstein, M., Petralia, R. S., Swanson, G. T., Wenthold, R. J. \& Heinemann, S. F. Distribution of kainate receptor subunits at hippocampal mossy fiber synapses. J. Neurosci. 23, 8013-8019 (2003).

40. Petralia, R. S., Wang, Y. X. \& Wenthold, R. J. Histological and ultrastructural localization of the kainate receptor subunits, KA2 and GluR6/7, in the rat nervous system using selective antipeptide antibodies. J. Comp. Neurol. 349, 85-110 (1994).

41. Biesemann, C. et al. Proteomic screening of glutamatergic mouse brain synaptosomes isolated by fluorescence activated sorting. EMBO J. 33, 157-170 (2014).

42. Pieribone, V. A. et al. Expression of synapsin III in nerve terminals and neurogenic regions of the adult brain. J. Comp. Neurol. 454, 105-114 (2002)

43. Schlüter, O. M. et al. Rabphilin knock-out mice reveal that rabphilin is not required for rab3 function in regulating neurotransmitter release. J. Neurosci. 19, 5834-5846 (1999).

44. Wenzel, H. J., Cole, T. B., Born, D. E., Schwartzkroin, P. A. \& Palmiter, R. D Ultrastructural localization of zinc transporter-3 ( $\mathrm{ZnT}-3)$ to synaptic vesicle membranes within mossy fiber boutons in the hippocampus of mouse and monkey. Proc. Natl Acad. Sci. USA 94, 12676-12681 (1997).

45. Straub, C. et al. Distinct subunit domains govern synaptic stability and specificity of the kainate receptor. Cell Rep. 16, 531-544 (2016).

46. Shigemoto, R. et al. Differential presynaptic localization of metabotropic glutamate receptor subtypes in the rat hippocampus. J. Neurosci. 17, 7503-7522 (1997).

47. Spangler, S. A. et al. Differential expression of liprin- $\alpha$ family proteins in the brain suggests functional diversification. J. Comp. Neurol. 519, 3040-3060 (2011).

48. Sadakata, T. et al. Differential distributions of the $\mathrm{Ca}^{2+}$-dependent activator protein for secretion family proteins (CAPS2 and CAPS1) in the mouse brain. J. Comp. Neurol. 495, 735-753 (2006).

49. Koopmans, F. et al. SynGO: an evidence-based, expert-curated knowledge base for the synapse. Neuron 103, 217-234.e4 (2019).

50. Katafuchi, T., Li, A. J., Hirota, S., Kitamura, Y. \& Hori, T. Impairment of spatial learning and hippocampal synaptic potentiation in c-kit mutant rats. Learn. Mem. 7, 383-392 (2000).

51. Kondo, T., Katafuchi, T. \& Hori, T. Stem cell factor modulates paired-pulse facilitation and long-term potentiation in the hippocampal mossy fiber-CA3 pathway in mice. Brain Res. 946, 179-190 (2002).

52. Motro, B., Wojtowicz, J. M., Bernstein, A. \& van der Kooy, D. Steel mutant mice are deficient in hippocampal learning but not long-term potentiation. Proc. Natl Acad. Sci. USA 93, 1808-1813 (1996).

53. Ozgul, S. et al. An ELISA-based screening platform for ligand-receptor discovery. Meth. Enzymol. 615, 453-475 (2019).

54. Wojtowicz, W. M. et al. A vast repertoire of Dscam binding specificities arises from modular interactions of variable Ig domains. Cell 130, 1134-1145 (2007).

55. Südhof, T. C. Synaptic neurexin complexes: a molecular code for the logic of neural circuits. Cell 171, 745-769 (2017).

56. Ranaivoson, F. M. et al. A proteomic screen of neuronal cell-surface molecules reveals IgLONs as structurally conserved interaction modules at the synapse. Structure 27, 893-906.e9 (2019). 
57. Boucard, A. A., Maxeiner, S. \& Südhof, T. C. Latrophilins function as heterophilic cell-adhesion molecules by binding to teneurins: regulation by alternative splicing. J. Biol. Chem. 289, 387-402 (2014).

58. O'Sullivan, M. L. et al. FLRT proteins are endogenous latrophilin ligands and regulate excitatory synapse development. Neuron 73, 903-910 (2012).

59. Silva, J.-P. et al. Latrophilin 1 and its endogenous ligand Lasso/teneurin-2 form a high-affinity transsynaptic receptor pair with signaling capabilities. Proc. Natl Acad. Sci. USA 108, 12113-12118 (2011).

60. Savas, J. N. et al. Ecto-Fc MS identifies ligand-receptor interactions through extracellular domain Fc fusion protein baits and shotgun proteomic analysis. Nat. Protoc. 9, 2061-2074 (2014).

61. Brenneke, F., Bukalo, O., Dityatev, A. \& Lie, A. A. Mice deficient for the extracellular matrix glycoprotein tenascin-r show physiological and structural hallmarks of increased hippocampal excitability, but no increased susceptibility to seizures in the pilocarpine model of epilepsy. Neuroscience 124, 841-855 (2004).

62. Nikonenko, A., Schmidt, S., Skibo, G., Brückner, G. \& Schachner, M. Tenascin-R-deficient mice show structural alterations of symmetric perisomatic synapses in the CA1 region of the hippocampus. J. Comp. Neurol. 456, 338-349 (2003)

63. Saghatelyan, A. K. et al. Reduced perisomatic inhibition, increased excitatory transmission, and impaired long-term potentiation in mice deficient for the extracellular matrix glycoprotein tenascin-R. Mol. Cell. Neurosci. 17, 226-240 (2001).

64. Inoue, N., Nishikawa, T., Ikawa, M. \& Okabe, M. Tetraspanin-interacting protein IGSF8 is dispensable for mouse fertility. Fertil. Steril. 98, 465-470 (2012).

65. Fehon, R. G., McClatchey, A. I. \& Bretscher, A. Organizing the cell cortex: the role of ERM proteins. Nat. Rev. Mol. Cell Biol. 11, 276-287 (2010).

66. Paglini, G., Kunda, P., Quiroga, S., Kosik, K. \& Cáceres, A. Suppression of radixin and moesin alters growth cone morphology, motility, and process formation in primary cultured neurons. J. Cell Biol. 143, 443-455 (1998).

67. Haas, M. A., Vickers, J. C. \& Dickson, T. C. Rho kinase activates ezrin-radixinmoesin (ERM) proteins and mediates their function in cortical neuron growth, morphology and motility in vitro. J. Neurosci. Res. 85, 34-46 (2007).

68. Henze, D. A., McMahon, D. B. T., Harris, K. M. \& Barrionuevo, G. Giant miniature EPSCs at the hippocampal mossy fiber to CA3 pyramidal cell synapse are monoquantal. J. Neurophysiol. 87, 15-29 (2002).

69. Poulopoulos, A. et al. Subcellular transcriptomes and proteomes of developing axon projections in the cerebral cortex. Nature 565, 356-360 (2019).

70. Pfeffer, M. et al. Synaptic and supra-synaptic organisation of the dopaminergic projection to the striatum. Preprint at https://doi.org/10.1101/ 2020.02.18.952978 (2020)

71. Micheva, K. D., Busse, B., Weiler, N. C., O’Rourke, N. \& Smith, S. J. Singlesynapse analysis of a diverse synapse population: proteomic imaging methods and markers. Neuron 68, 639-653 (2010).

72. Zhu, F. et al. Architecture of the mouse brain synaptome. Neuron 99, 781-799. e10 (2018).

73. Cizeron, M. et al. A brainwide atlas of synapses across the mouse life span. Science 369, 270-275 (2020).

74. Selimi, F., Cristea, I. M., Heller, E., Chait, B. T. \& Heintz, N. Proteomic studies of a single CNS synapse type: the parallel fiber/purkinje cell synapse. PLoS Biol. 7, e83 (2009).

75. Li, J. et al. Cell-surface proteomic profiling in the fly brain uncovers wiring regulators. Cell 180, 373-386.e15 (2020).

76. Uezu, A. et al. Identification of an elaborate complex mediating postsynaptic inhibition. Science 353, 1123-1129 (2016).

77. Loh, K. H. et al. Proteomic analysis of unbounded cellular compartments: synaptic clefts. Cell 166, 1295-1307.e21 (2016).

78. Schreiner, D., Savas, J. N., Herzog, E., Brose, N. \& de Wit, J. Synapse biology in the 'circuit-age'-paths toward molecular connectomics. Curr. Opin. Neurobiol. 42, 102-110 (2017)

79. Gerber, H. et al. The APMAP interactome reveals new modulators of APP processing and beta-amyloid production that are altered in Alzheimer's disease. Acta Neuropathol. Commun. 7, 13 (2019).

80. Mosser, S. et al. The adipocyte differentiation protein APMAP is an endogenous suppressor of $\mathrm{A} \beta$ production in the brain. Hum. Mol. Genet. 24, 371-382 (2015).

81. Rasila, T. et al. Astroprincin (FAM171A1, C10orf38): a regulator of human cell shape and invasive growth. Am. J. Pathol. 189, 177-189 (2019).

82. Kawano, H. et al. Identification and characterization of novel developmentally regulated neural-specific proteins, BRINP family. Brain Res. Mol. Brain Res. 125, 60-75 (2004)

83. Berkowicz, S. R., Featherby, T. J., Whisstock, J. C. \& Bird, P. I. Mice lacking Brinp2 or Brinp3, or both, exhibit behaviors consistent with neurodevelopmental disorders. Front. Behav. Neurosci. 10, 196 (2016).

84. Stipp, C. S., Kolesnikova, T. V. \& Hemler, M. E. EWI-2 is a major CD9 and CD81 partner and member of a novel Ig protein subfamily. J. Biol. Chem. 276, 40545-40554 (2001)
85. Stipp, C. S., Kolesnikova, T. V. \& Hemler, M. E. EWI-2 regulates alpha3beta1 integrin-dependent cell functions on laminin-5. J. Cell Biol. 163, 1167-1177 (2003).

86. Kolesnikova, T. V. et al. EWI-2 modulates lymphocyte integrin alpha4beta1 functions. Blood 103, 3013-3019 (2004)

87. Zhang, X. A., Lane, W. S., Charrin, S., Rubinstein, E. \& Liu, L. EWI2/PGRL associates with the metastasis suppressor KAI1/CD82 and inhibits the migration of prostate cancer cells. Cancer Res. 63, 2665-2674 (2003).

88. Yáñez-Mó, M., Barreiro, O., Gordon-Alonso, M., Sala-Valdés, M. \& SánchezMadrid, F. Tetraspanin-enriched microdomains: a functional unit in cell plasma membranes. Trends Cell Biol. 19, 434-446 (2009).

89. Galimberti, I. et al. Long-term rearrangements of hippocampal mossy fiber terminal connectivity in the adult regulated by experience. Neuron $\mathbf{5 0}$, 749-763 (2006).

90. Sato, Y. et al. Cartilage acidic protein-1B (LOTUS), an endogenous Nogo receptor antagonist for axon tract formation. Science 333, 769-773 (2011).

91. Demyanenko, G. P. et al. Neural cell adhesion molecule NrCAM regulates Semaphorin 3F-induced dendritic spine remodeling. J. Neurosci. 34, 11274-11287 (2014)

92. Morita, A. et al. Regulation of dendritic branching and spine maturation by semaphorin3A-Fyn signaling. J. Neurosci. 26, 2971-2980 (2006).

93. Tran, T. S. et al. Secreted semaphorins control spine distribution and morphogenesis in the postnatal CNS. Nature 462, 1065-1069 (2009).

94. Contractor, A. et al. Trans-synaptic Eph receptor-ephrin signaling in hippocampal mossy fiber LTP. Science 296, 1864-1869 (2002).

95. Galimberti, I., Bednarek, E., Donato, F. \& Caroni, P. EphA4 signaling in juveniles establishes topographic specificity of structural plasticity in the hippocampus. Neuron 65, 627-642 (2010).

96. Zhang, P. et al. Heparan sulfate organizes neuronal synapses through neurexin partnerships. Cell 174, 1450-1464.e23 (2018).

97. Matsuda, K. et al. Transsynaptic modulation of kainate receptor functions by Clq-like proteins. Neuron 90, 752-767 (2016)

98. Toni, N. et al. Neurons born in the adult dentate gyrus form functional synapses with target cells. Nat. Neurosci. 11, 901-907 (2008).

99. Rice, H. C. et al. Pancortins interact with amyloid precursor protein and modulate cortical cell migration. Development 139, 3986-3996 (2012).

100. Chen, N. et al. Interaction proteomics reveals brain region-specific AMPA receptor complexes. J. Proteome Res. 13, 5695-5706 (2014).

101. von Engelhardt, J. et al. CKAMP44: a brain-specific protein attenuating shortterm synaptic plasticity in the dentate gyrus. Science 327, 1518-1522 (2010).

102. Schwenk, J. et al. High-resolution proteomics unravel architecture and molecular diversity of native AMPA receptor complexes. Neuron 74, 621-633 (2012).

103. Shanks, N. F. et al. Differences in AMPA and kainate receptor interactomes facilitate identification of AMPA receptor auxiliary subunit GSG1L. Cell Rep. 1, 590-598 (2012)

104. Pandya, N. J. et al. Noelin1 affects lateral mobility of synaptic AMPA receptors. Cell Rep. 24, 1218-1230 (2018).

105. Hashimoto, T., Yamada, M., Maekawa, S., Nakashima, T. \& Miyata, S. IgLON cell adhesion molecule Kilon is a crucial modulator for synapse number in hippocampal neurons. Brain Res. 1224, 1-11 (2008).

106. Ray, A. \& Treloar, H. B. IgSF8: a developmentally and functionally regulated cell adhesion molecule in olfactory sensory neuron axons and synapses. Mol. Cell. Neurosci. 50, 238-249 (2012).

107. Usardi, A., Iyer, K., Sigoillot, S. M., Dusonchet, A. \& Selimi, F. The immunoglobulin-like superfamily member IGSF3 is a developmentally regulated protein that controls neuronal morphogenesis. Dev. Neurobiol. 77, 75-92 (2017)

108. Cijsouw, T. et al. Mapping the proteome of the synaptic cleft through proximity labeling reveals new cleft proteins. Proteomes 6, 48 (2018).

109. Sala-Valdés, M. et al. EWI-2 and EWI-F link the tetraspanin web to the actin cytoskeleton through their direct association with ezrin-radixin-moesin proteins. J. Biol. Chem. 281, 19665-19675 (2006).

110. Gordón-Alonso, M. et al. EWI-2 association with $\alpha$-actinin regulates T cell immune synapses and HIV viral infection. J. Immunol. 189, 689-700 (2012).

111. Antoine-Bertrand, J., Ghogha, A., Luangrath, V., Bedford, F. K. \& Lamarche-Vane, N. The activation of ezrin-radixin-moesin proteins is regulated by netrin-1 through Src kinase and RhoA/Rho kinase activities and mediates netrin-1-induced axon outgrowth. Mol. Biol. Cell 22, 3734-3746 (2011).

112. Marsick, B. M., San Miguel-Ruiz, J. E. \& Letourneau, P. C. Activation of ezrin/ radixin/moesin mediates attractive growth cone guidance through regulation of growth cone actin and adhesion receptors. J. Neurosci. 32, 282-296 (2012)

113. Zacharias, U., Leuschner, R., Nörenberg, U. \& Rathjen, F. G. Tenascin-R induces actin-rich microprocesses and branches along neurite shafts. Mol. Cell. Neurosci. 21, 626-633 (2002).

114. Jonas, P. \& Lisman, J. Structure, function, and plasticity of hippocampal dentate gyrus microcircuits. Front. Neural Circuits 8, 107 (2014). 
115. Ribeiro, L. F. et al. SorCS1-mediated sorting in dendrites maintains neurexin axonal surface polarization required for synaptic function. PLoS Biol. 17, e3000466 (2019).

116. He, L., Diedrich, J., Chu, Y.-Y. \& Yates, J. R. Extracting accurate precursor information for tandem mass spectra by RawConverter. Anal. Chem. 87, 11361-11367 (2015).

117. Eng, J. K., McCormack, A. L. \& Yates, J. R. An approach to correlate tandem mass spectral data of peptides with amino acid sequences in a protein database. J. Am. Soc. Mass Spectrom. 5, 976-989 (1994).

118. Xu, T. et al. ProLuCID: An improved SEQUEST-like algorithm with enhanced sensitivity and specificity. J. Proteom. 129, 16-24 (2015).

119. Cociorva, D., Tabb, D. L. \& Yates, J. R. Validation of tandem mass spectrometry database search results using DTASelect. Curr. Protoc. Bioinformatics Chapter 13, Unit 13.4 (2007).

120. Tabb, D. L., McDonald, W. H. \& Yates, J. R. DTASelect and contrast: tools for assembling and comparing protein identifications from shotgun proteomics. J. Proteome Res. 1, 21-26 (2002).

121. Florens, L. et al. Analyzing chromatin remodeling complexes using shotgun proteomics and normalized spectral abundance factors. Methods 40, 303-311 (2006).

122. Zybailov, B. et al. Statistical analysis of membrane proteome expression changes in Saccharomyces cerevisiae. J. Proteome Res. 5, 2339-2347 (2006).

123. Mi, H. et al. Protocol update for large-scale genome and gene function analysis with the PANTHER classification system (v.14.0). Nat. Protoc. 14, 703-721 (2019).

124. Carlin, R. K., Grab, D. J., Cohen, R. S. \& Siekevitz, P. Isolation and characterization of postsynaptic densities from various brain regions: enrichment of different types of postsynaptic densities. J. Cell Biol. 86, 831-845 (1980).

125. Schindelin, J. et al. Fiji: an open-source platform for biological-image analysis. Nat. Methods 9, 676-682 (2012).

126. Mattis, J. et al. Principles for applying optogenetic tools derived from direct comparative analysis of microbial opsins. Nat. Methods 9, 159-172 (2011).

127. de Wit, J. et al. LRRTM2 interacts with Neurexin 1 and regulates excitatory synapse formation. Neuron 64, 799-806 (2009).

128. de Wit, J. et al. Unbiased discovery of glypican as a receptor for LRRTM4 in regulating excitatory synapse development. Neuron 79, 696-711 (2013).

\section{Acknowledgements}

We thank Franck Polleux, Dietmar Schmucker, Pierre Vanderhaeghen, Anirvan Ghosh, Dan Dascenco, Luís Ribeiro, and Sara Calafate for critical reading of the manuscript, and De Wit lab members for helpful discussion and comments. We thank Pier Andrée Penttila and Christèle Nkama (VIB-KU Leuven FACS Core), the VIB-KU Leuven BioImaging Core, Joris Vandenbempt, and Brenda Luong for experimental help; Nicola Fattorelli for data visualization advice; Etienne Herzog and Matthew Holt for experimental advice. Leica SP8x confocal microscope was provided by InfraMouse (KU Leuven-VIB) through a Hercules type 3 project (ZW09-03). N.A. is supported by the Fundação para a Ciência e a Tecnologia (FCT, Grant Number SFRH/BD/128869/2017). D.C. is supported by NSF IOS Grant \#1755189, RWJ Foundation Grant \#74260, and Research for Life (WMRF) 2019/301. J.N.S. is supported by R01AG061787. J.d.W is supported by ERC Starting Grant (\#311083), FWO Odysseus Grant, FWO Project Grant G0C4518N, FWO EOS Grant G0H2818N, and Methusalem Grant of KU Leuven/Flemish Government.

\section{Author contributions}

N.A., J.N.S., and J.d.W. conceived the study and designed experiments. N.A., S.N.S., J.V., G.C., V.R., J.t.B., L.T., S.P., K.M.V., N.V.G., D.C., and K.D.W. performed experiments and analyzed data. N.A. and J.d.W. wrote the paper with input from all authors. All authors contributed to and approved the final version.

\section{Competing interests}

The authors declare no competing interests.

\section{Additional information}

Supplementary information is available for this paper at https://doi.org/10.1038/s41467020-18956-x.

Correspondence and requests for materials should be addressed to J.N.S. or J.d.W.

Peer review information Nature Communications thanks Seth Grant, Tabrez Siddiqui and the other anonymous reviewer(s) for their contribution to the peer review of this work. Peer reviewer reports are available.

Reprints and permission information is available at http://www.nature.com/reprints

Publisher's note Springer Nature remains neutral with regard to jurisdictional claims in published maps and institutional affiliations.

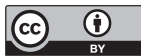

Open Access This article is licensed under a Creative Commons Attribution 4.0 International License, which permits use, sharing, adaptation, distribution and reproduction in any medium or format, as long as you give appropriate credit to the original author(s) and the source, provide a link to the Creative Commons license, and indicate if changes were made. The images or other third party material in this article are included in the article's Creative Commons license, unless indicated otherwise in a credit line to the material. If material is not included in the article's Creative Commons license and your intended use is not permitted by statutory regulation or exceeds the permitted use, you will need to obtain permission directly from the copyright holder. To view a copy of this license, visit http://creativecommons.org/ licenses/by/4.0/

(C) The Author(s) 2020 